Environmental Restoration Division ORNL Environmental Restoration Program

\title{
Waste Characterization Data Manual for the Inactive Liquid Low-Level Radioactive Waste Tank Systems at Oak Ridge National Laboratory, Oak Ridge, Tennessee
}

Date Issued-June 1992

\author{
Prepared by \\ Engineering Division \\ Oak Ridge National Laboratory
}

Prepared for U.S. Department of Energy

Office of Environmental Restoration and Waste Management under budget and reporting code EW 20

OAK RIDGE NATIONAL LABORATORY

Oak Ridge, Tennessee 37831-6285 managed by MARTIN MARIETTA ENERGY SYSTEMS, INC.

for the

U.S. DEPARTMENT OF ENERGY under contract DE-AC05-84OR21400 


\section{CONTENTS}

ACRONYMS $\ldots \ldots \ldots \ldots \ldots \ldots \ldots \ldots \ldots \ldots \ldots \ldots \ldots$

EXECUTIVE SUMMARY $\ldots \ldots \ldots \ldots \ldots \ldots \ldots \ldots \ldots \ldots \ldots \ldots$ vii

1. INTRODUCTION

1.1 BACKGROUND

1.2 PURPOSE

1.3 SCOPE

1.4 DEFINITIONS

1.5 SAMPLING OF CONTENTS FOR CATEGORY D LLLW TANK SYSTEMS

1.6 ANALYTICAL METHODS

1.6.1 Volatile Organic Compound Analysis

1.6.2 Semivolatile Organic Compound Analysis

1.6.3 Polychlorinated Biphenyl Analysis

1.6.4 Radiochemical and Inorganic Compound Analysis

1.7 REFERENCES

2. WASTE CHARACTERIZATION SUMMARY FOR THE ORNL CATEGORY D LLLW TANKS

$\begin{array}{lccc} & \text { Tank No. } & \text { Rev } & \text { Date Issued } \\ 2.1 & \text { W-5 } & 0 & 6 / 92 \\ 2.2 & \text { W-6 } & 0 & 6 / 92 \\ 2.3 & \text { W-7 } & 0 & 6 / 92 \\ 2.4 & \text { W-8 } & 0 & 6 / 92 \\ 2.5 & \text { W-9 } & 0 & 6 / 92 \\ 2.6 & \text { W-10 } & 0 & 6 / 92 \\ 2.7 & \text { W-11 } & 0 & 6 / 92 \\ 2.8 & \text { T-1 } & 0 & 6 / 92 \\ 2.9 & \text { T-2 } & 0 & 6 / 92 \\ 2.10 & \text { T-3 } & 0 & 6 / 92 \\ & & & \\ 2.11 & \text { T-4 } & 0 & 6 / 92 \\ 2.12 & \text { T-9 } & 0 & 6 / 92 \\ 2.13 & \text { TH-1 } & 0 & 6 / 92 \\ 2.14 & \text { TH-2 } & 0 & 6 / 92 \\ 2.15 & \text { TH-3 } & 0 & 6 / 92 \\ 2.16 & \text { TH-4 } & 0 & 6 / 92 \\ 2.17 & \text { WC-1 } & 0 & 6 / 92 \\ 2.18 & \text { WC-15 } & 0 & 6 / 92 \\ 2.19 & \text { WC-17 } & 0 & 6 / 92 \\ 2.20 & \text { W-1 } & 0 & 6 / 92\end{array}$




\begin{tabular}{|c|c|c|c|}
\hline & Tank No. & $\operatorname{Rev}$ & Date Issued \\
\hline 2.21 & $W-1 A$ & 0 & $6 / 92$ \\
\hline 2.22 & $W-2$ & 0 & $6 / 92$ \\
\hline 2.23 & $W-3$ & 0 & $6 / 92$ \\
\hline 2.24 & W-4 & 0 & $6 / 92$ \\
\hline 2.25 & W.13 & 0 & $6 / 92$ \\
\hline 2.26 & W-14 & 0 & $6 / 92$ \\
\hline 2.27 & W-15 & 0 & $6 / 92$ \\
\hline 2.28 & 7560 & 0 & $6 / 92$ \\
\hline 2.29 & 7562 & 0 & $6 / 92$ \\
\hline 2.30 & $\mathrm{~T}-30$ & 0 & $6 / 92$ \\
\hline 2.31 & W-19 & 0 & $6 / 92$ \\
\hline 2.32 & W-20 & 0 & $6 / 92$ \\
\hline 2.33 & $3001-B$ & & TBD \\
\hline 2.34 & 3003-A & & TBD \\
\hline 2.35 & $3004-B$ & & TBD \\
\hline 2.36 & 3013 & & TBD \\
\hline 2.37 & H-209 & & TBD \\
\hline 2.38 & 7503-A & & TBD \\
\hline 2.39 & $3001-S$ & & TBD \\
\hline 2.40 & $3002-A$ & & TBD \\
\hline 2.41 & 4501-P & & TBD \\
\hline 2.42 & S. .424 & & TBD \\
\hline 2.43 & $\mathrm{~T}-14$ & & TBD \\
\hline 2.44 & W-1I & & TBD \\
\hline 2.45 & WC-4 & & TBD \\
\hline 2.46 & W-17 & & TBD \\
\hline 2.47 & W-18 & & TBD \\
\hline 2.48 & WC-5 & & TBD \\
\hline 2.49 & WC-6 & & TBD \\
\hline 2.50 & WC-8 & & TBD \\
\hline 2.51 & WC-11 & & TBD \\
\hline 2.52 & WC-12 & & TBD \\
\hline 2.53 & WC-13 & & TBD \\
\hline 2.54 & WC-14 & & TBD \\
\hline
\end{tabular}




\section{ACRONYMS}

$\begin{array}{ll}\text { AA } & \text { atomic absorption } \\ \text { CLP } & \text { Contract Laboratory Procedure } \\ \text { CRQL } & \text { contract required detection limit } \\ \text { DAI-GC } & \text { direct aqueous injection-gas chromatography } \\ \text { EPA } & \text { U. S. Environmental Protection Agency } \\ \text { EP-TOX } & \text { Extraction Procedure Toxicity } \\ \text { ER } & \text { Environmental Restoration } \\ \text { FFA } & \text { Federal Facility Agreement } \\ \text { GC-MS } & \text { gas chromatography-mass spectrometry } \\ \text { HRE } & \text { Homogenous Reactor Experiment } \\ \text { HRLAL } & \text { High Radiation Level Analytical Laboratory } \\ \text { ICP } & \text { inductively coupled plasma } \\ \text { LLLW } & \text { liquid low-level radioactive waste } \\ \text { OHF } & \text { Old Hydrofracture Facility } \\ \text { ORNL } & \text { Oak Ridge National Laboratory } \\ \text { PAH } & \text { polycyclic aromatic hydrocarbon } \\ \text { PCB } & \text { polychlorinated biphenyl } \\ \text { QA } & \text { quality assurance } \\ \text { RCRA } & \text { Resource Conservation and Recovery Act } \\ \text { SVOA } & \text { semivolatile organic analysis } \\ \text { TBP } & \text { tributylphosphate } \\ \text { TCL } & \text { Target Compound List } \\ \text { TDEC } & \text { Tennessee Department of Environment and Conservation } \\ \text { TIC } & \text { tentatively identified compound } \\ \text { TOC } & \text { total organic carbon } \\ \text { VOA } & \text { volatile organic analysis } \\ & \end{array}$

\section{DISCLAIMER}

\footnotetext{
This report was prepared as an account of work sponsored by an agency of the United States Government. Neither the United States Government nor any agency thereof, nor any of their employees, makes any warranty, express or implied, or assumes any legal liability or responsibility for the accuracy, completeness, or usefulness of any information, apparatus, product, or process disclosed, or represents that its use would not infringe privately owned rights. Reference herein to any specific commercial product, process, or service by trade name, tradernark, manufacturer, or otherwise does not necessarily constitute or imply its endorsement, recommendation, or favoring by the United States Government or any agency thereof. The views and opinions of authors expressed herein do not necessarily state or reflect those of the United States Government or any agency thereof.
} 


\section{EXECUTIVE SUMMARY}

This Waste Characterization Data Manual contains the results of an analysis of the contents of liquid low-level waste (LLLW) tanks that have been removed from service in accordance with the requirements of the Oak Ridge Reservation (ORR) Federal Facility Agreement (FFA), Sect. IX.G.1.

Pursuant to the provisions of the Comprehensive Environmental Response, Compensation, and Liability Act, as amended by the Superfund Amendment and Reauthorization Act, the ORR, consisting of $-58,000$ acres owned by the United States and under the jurisdiction of the U. S. Department of Energy (DOE), was placed on the National Priorities List on December 21, 1989. An FFA for the ORR, which was signed by the U. S. Environmental Protection Agency (EPA), Region IV; DOE; and the Tennessee Department of Environment and Conservation (TDEC), became effective January 1, 1992. The objective of the agreement is to ensure that environmental impacts resulting from operations at the ORR, both present and past, are thoroughly investigated and remediated to protect the public health, welfare, and environment.

The FFA (Sect. IX.G.1) requires that waste characterizations be conducted and provided to EPA and TDEC for all LLLW tanks that are removed from service. These waste characterizations will include the results of sampling and analysis of the tank contents, including wastes, liquids, and sludges.

This manual contains the results of sampling activities that were conducted at the Oak Ridge National Laboratory in 1988. Thirty-three tanks were sampled and analyzed at that time. Sampling of the remaining inactive tanks is currently underway, and data from these tanks will be added to this manual as they become available. Data are presented from analysis of volatile organic compounds, semivolatile organic compounds, polychlorinated biphenyls, radiochemical compounds, and inorganic compounds.

Most of the liquid samples were collected with a small vacuum pump. Although it could volatize the lighter organics in the liquid, this procedure minimized exposure of collection personnel to radiation. Liquid samples were collected into $250-\mathrm{mL}$ glass sample jars with Teflon-lined caps. Each sample was given a unique identification number.

No standard EPA methods exist for the preparation and analysis of mixed wastes, and very few laboratories have experience in this area. Therefore, the organic chemical characterization in this project required that much of the methodology be developed as needed rather than during a preliminary development phase.

Quality assurance (QA) during tank sampling activities was maintained primarily through the use of tank-specific procedures for the sampling crew to follow. These procedures provided step-by-step instructions for the collection, labeling, and shipping of each sample. If any procedural changes were required, the Project Manager was notified, and approval was required from the appropriate levels of management before operations 
could begin. Independent surveillance by the QA Specialist verified that sampling procedures, chain-of-custody criteria, radiation protection, and operator training were in compliance with all written procedures. Sampling activities that followed EPA procedures met analysis-level-III quality requirements. Analyses that required modifications of EPA procedures met analysis-level-V quality requirements.

The sample results are organized in this manual by individual tanks. Data reporting conventions and notations are defined in Sect. 2. 


\section{INTRODUCTION}

\subsection{BACKGROUND}

Oak Ridge National Laboratory (ORNL) is a multidisciplinary research facility that began operation in 1943 as part of the Manhattan Project. The original mission of ORNL was to develop a prototype graphite reactor and to reprocess the reactor fuel for plutonium recovery. After World War II, the primary functions of ORNL were fuel reprocessing research; radioisotopes production and applications development; and development, testing, and operation of nuclear risactor concepts. More recently, ORNL has increased its role in biological, environmertal, energy, and materials research. As a consequence of these multidisciplinary research activities, heterogeneous waste has been generated in varying amounts over time, including solid and liquid radioactive, hazardous, and mixed wastes.

Most of the liquid low-level waste (LLLW) system was installed more than 30 years ago. The initial system and its subsequent modifications were designed to minimize radiation exposure to LLLW system users and operators. System features include provisions for remote operations to minimize personnel exposure and unvalved, gravity-drained transfer lines to prevent waste backup into generator areas. Over the years, tank systems were abandoned as their integrity was breached or as programs were terminated. Some of the tanks were abandoned in place with liquid waste and sludge left in them. As-built drawings for most of the tank systems do not exist. The out-of-service portion of the system consists almost entirely of tanks without secondary containment. Figures 1.1 and 1.2 show the LLLW tank locations in Bethel Valley and Melton Valley, respectively.

\subsection{PURPOSE}

Section IX.G.1 of the Oak Ridge Reservation Federal Facility Agreement (FFA) requires the completion of waste characterizations for all out-of-service tank systems. Tank contents must be sampled and analyzed for hazardous and radioactive constituents and the data presented in the waste characterization. The purpose of this data manual is to present waste characterization data for the Category D LLLW tanks to the U.S. Environmental Protection Agency (EPA) and the Tennessee Department of Environment and Conservation (TDEC).

\subsection{SCOPE}

The methodology described in this manual and the data shown in Revision 0 of Sects. 2.1 through 2.32 have been extracted from ORNL reports published by Autrey et al. ${ }^{1,2}$ Data for the remaining Category $D$ tanks will be included as they become available. As additional tanks are accepted into the Environmental Restoration (ER) Program, their waste characterization data will also be incorporated into this manual. 


\subsection{DEFINITIONS}

Category D tank system. A singly contained LLLW tank system that is removed from service and will no longer receive program-generated waste.

LLLW. Hazardous, radioactive, and mixed liquid waste having an activity greater than the trace levels permitted in process wastes but less than $5 \mathrm{Ci} / g a l$ of beta-gamma emitters ( $52 \mathrm{Ci} / \mathrm{gal}$ by the revised waste acceptance criteria) and less than $100 \mathrm{nCi} / \mathrm{gal}$ of alphaemitting transuranic elements.

\subsection{SAMPLING OF CONTENTS FOR CATEGORY D LLLW TANK SYSTEMS}

Because of the physical design of the tanks (Fig. 1.3), sample collection could take place from only a very limited area within the tank. Sample quantities were also limited to minimize exposure of the field personnel to radiation while collecting the samples. However, because of the length of time the tank contents have had to settle, the liquid contents of most of the tanks were expected to be relatively homogeneous.

Most of the liquid samples were collected with a small vacuum pump. Although it could volatize the lighter organics in the liquid, this procedure minimized exposure of collection personnel to radiation. Liquid samples were collected into $250-\mathrm{mL}$ glass sample jars with Teflon-lined caps. Each sample was given a unique identification number.

Vertical stratification of the liquid was expected in several tanks; therefore, if liqvid depths were greater than $5 \mathrm{ft}$, samples were usually taken near the top, at the midpoint, and at the botiom of the liquid. If the liquid depth was $1-5 \mathrm{ft}$, samples were collected from the top and bottom. For very shallow liquid depths $(<1 \mathrm{ft})$, double samples were collected at only one depth.

Sludge has been found in a number of the tanks. The liquid-sludge interface in these tanks was located using the Markland model 10 sludge gun. This tool measures the amount of light transmitted across a fixed gap in the probe to detect changes in the percentage of solids in the liquid. As the probe enters the sludge, an alarm sounds, and the operator records the depth.

Based on earlier reports, soft and hard sludges were expected to be found in the tanks. Two different sludge collectors were prepared to accommodate the varying sludge conditions. The soft sludge collector has an open-ended sample collection tube at the bottom. After the sludge enters the tube, a flat, neoprene-coated plate is rotated over the opening. The hard sludge collector uses a beveled cutting edge to core samples as it is driven into the sludge. The collector was not used on steel tanks because of the possibility of puncturing the tank shell. All sludge was cored, and the samples were homogenized before they were analyzed. 


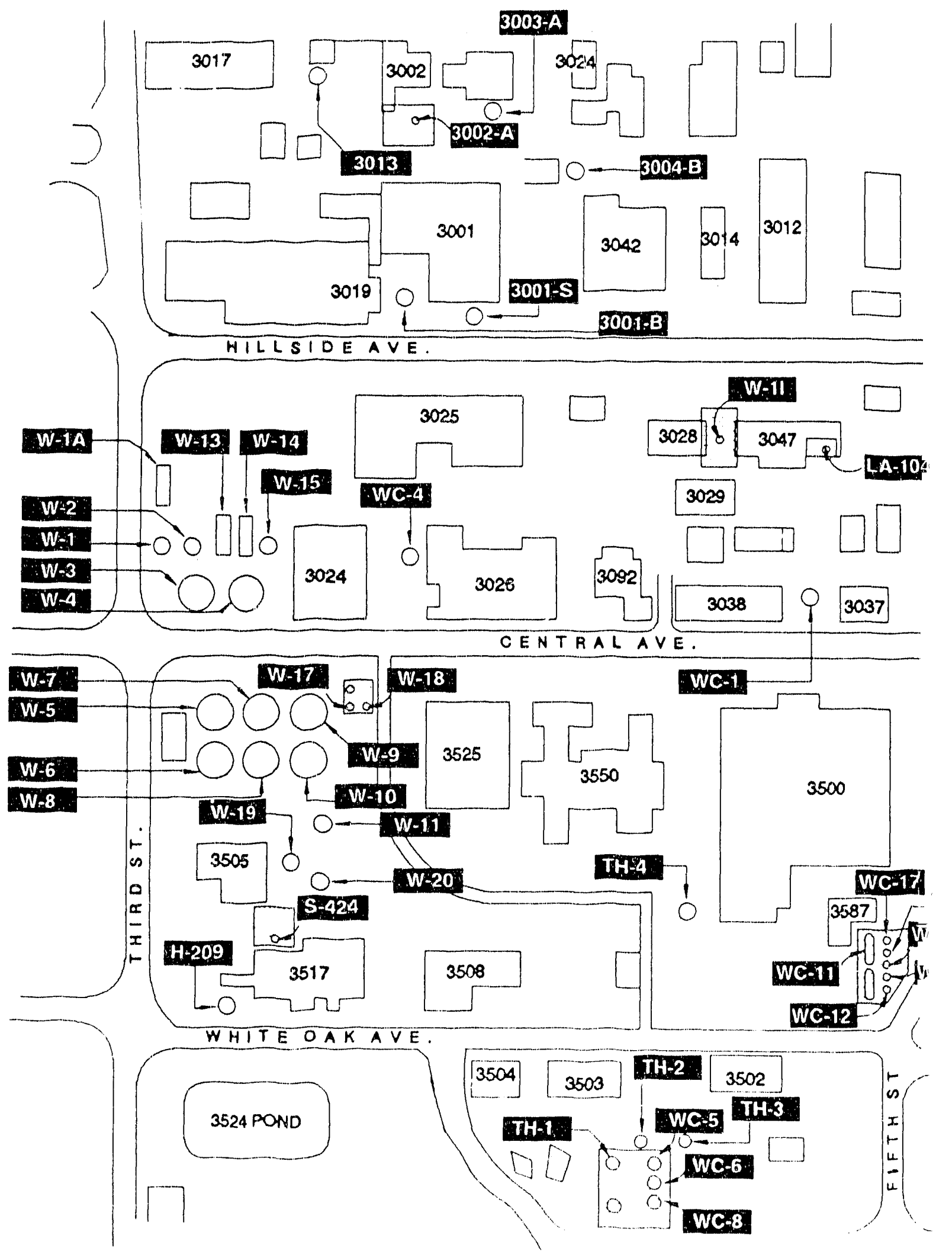

Fig. 1.1. Location of inactive LLLLW tanks ir 


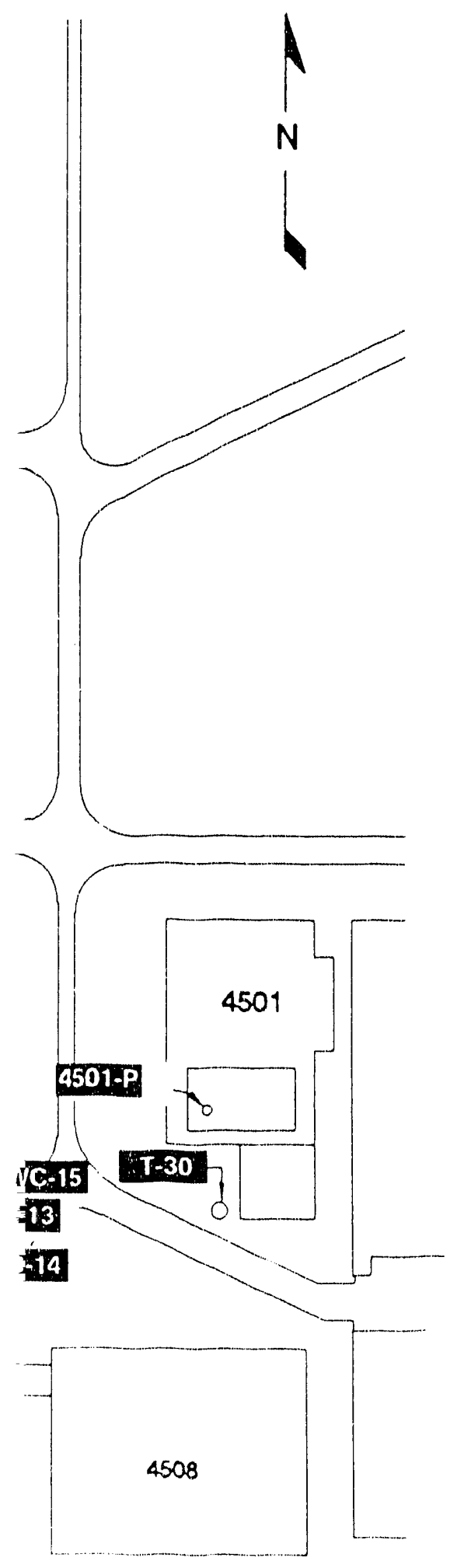

Key:

T-30. - Indicales LILW Tank Numbers

the Bethel Valley main laboratory area. 
Waste Characterization Data for ORNL Inactive LLLW Tanks

Rev. 0 / Junle 1992

Section $1 /$ page 5 of 13

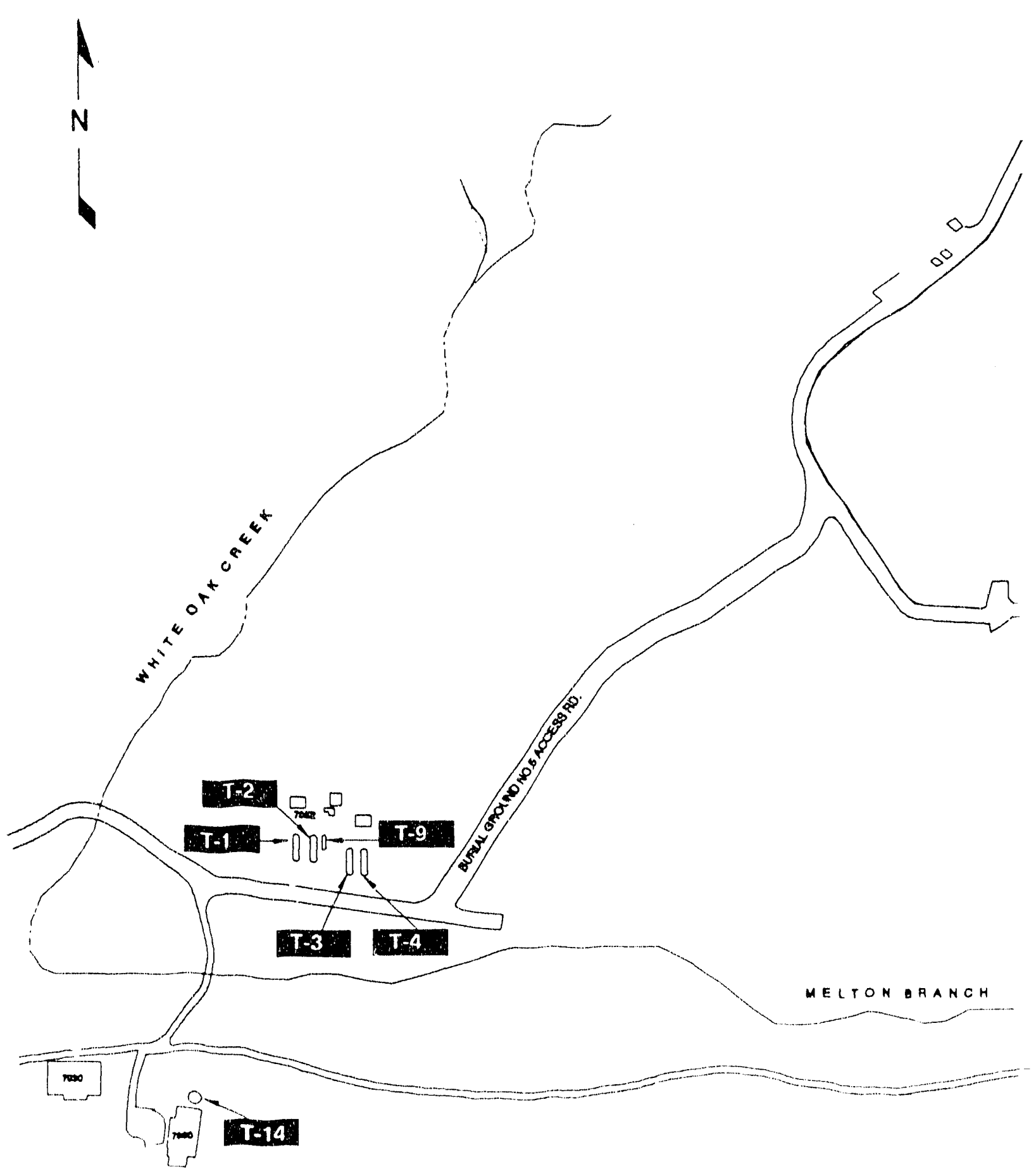

Fig. 1.2. Lucation of inactive LLLW 


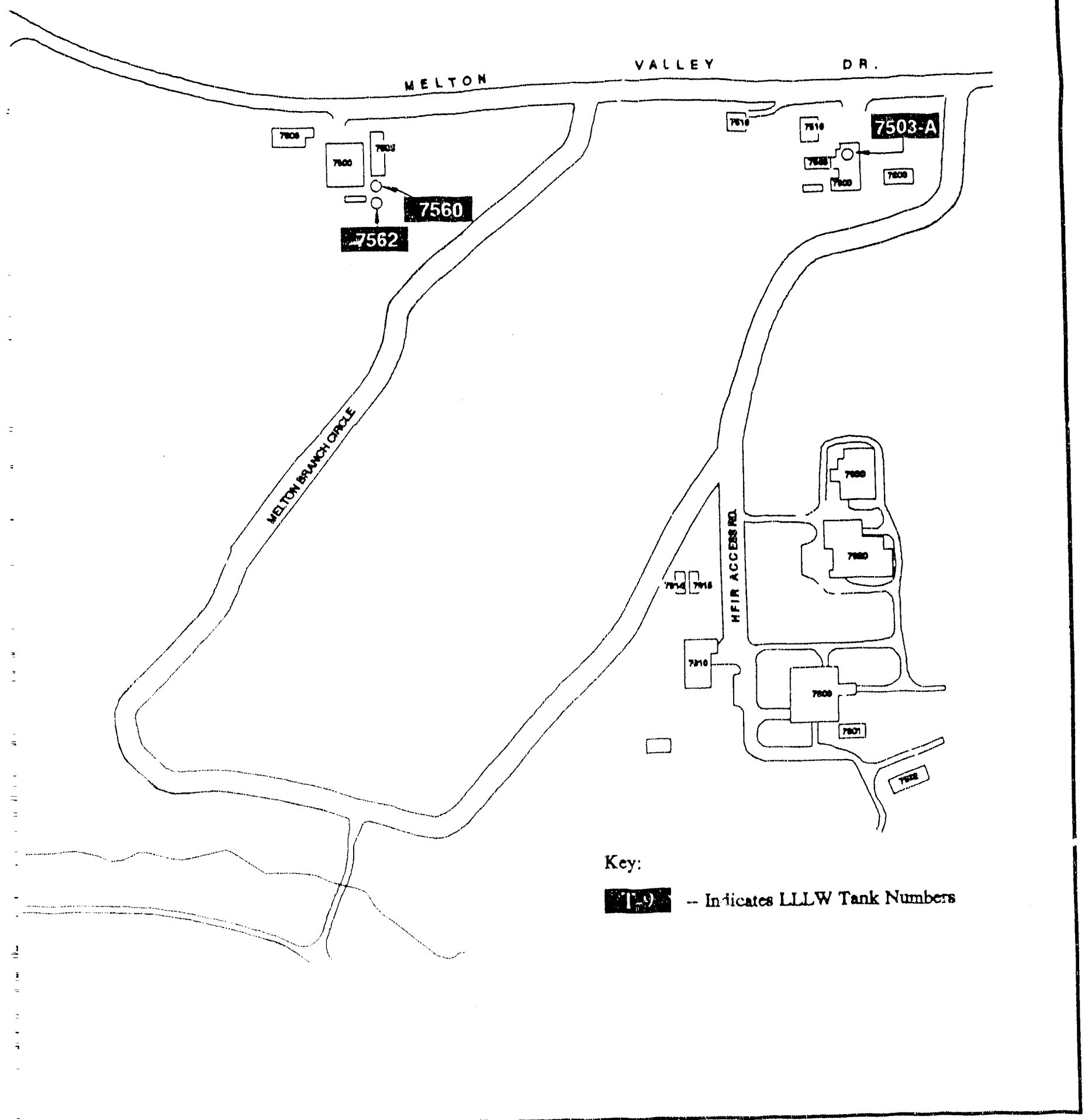

$=$ tanks in the Melton Valley area.
$=$ 


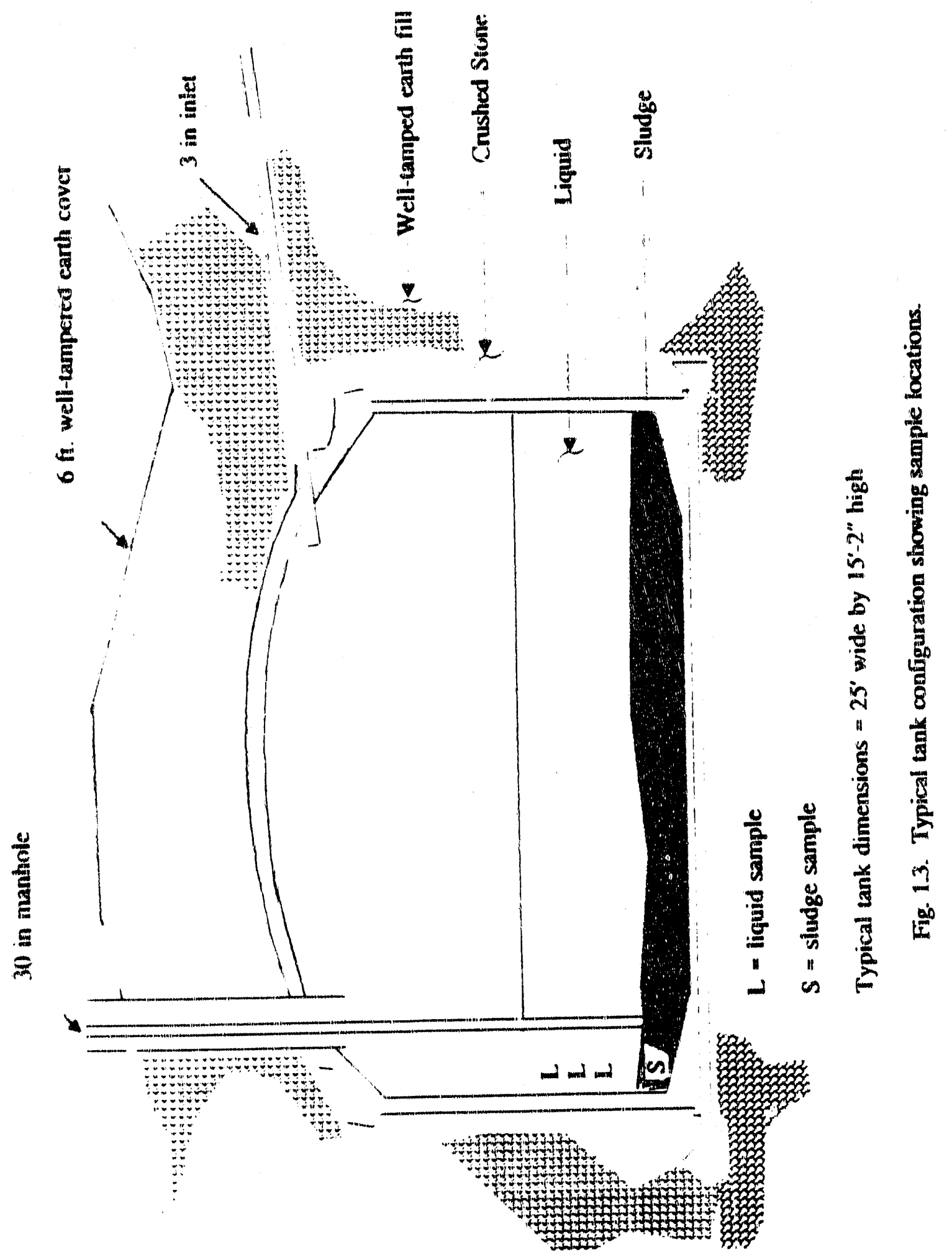


Quality assurance (QA) during tank sampling activities was maintained primarily through the use of tank-specific proc dures for the sampling crew to follow. These procedures provided step-by-step instructions for the collection, labeling, and shipping of each sample. If any procedural changes were required, the Project Manager was notified, and approval was required from the appropriate levels of management before operations could begin. An independent surveillance by the QA Specialist verified that sampling procedures, chain-ofcustody criteria, radiation protection, and operator training were in compliance with all written procedures. Sampling activities that followed EPA procedures met analysis-level-III quality requirements. Analyses that required modifications of EPA procedures met analysislevel-V quality requirements.

All field data, such as liquid and sludge depths, sample identification numbers, location of the sampling port on the tank, and field surveys, were recorded on data forms to ensure an accurate account of the sampling activities. Chain-of-custody forms were used to track individual samples from their collection point to the individual laboratories conducting the analyses.

\subsection{ANALYTICAL METHODS}

No standard EPA methods exist for the preparation and analysis of mixed wastes, and very few laboratories have experience in this area. Therefore, the organic chemical characterization in this project required that much of the methodology be developed as needed rather than during a preliminary development phase.

The approach taken for the determination of regulated organic compounds in the samples collected from the inactive tanks was to prepare extracts in (radioactive) contamination-zoned facilities and to perform the analytical measurements in analytical laboratories. The preparation methodologies followed EPA SW $-846^{3}$ or Contract Laboratory Program (CLP) methodology ${ }^{4}$ as closely as possible. Method deviations required by the radioactive nature or other characteristics of the samples were documented. These deviations are explained in the ORNL report published by Autry et al. ${ }^{1}$ The quantitative measurements were conducted by EPA CLP methods.

The staff of the High Radiation Level Analytical Laboratory (HRLAL) received and logged in the samples and transferred aliquots to the Separations and Synthesis Group for organic analytical preparation. Except for the direct aqueous injection-gas chromatographic (DAI-GC) determination of major volatile urganic compounds, all the quantitative measurements were performed by the Organic Analysis Group. All sample transfers were accompanied by chain-of-custody documentation.

The CL.P analyses were specifically calibrated for the EPA CLP Target Compound List (TCL). Because EPA Region IV staff suggested analyzing for as much of the 40 CFR 261, Appendix VIII, list as possible within the constraints of worker protection, the detection of such compounds as tentatively identified compounds (TICs) was also investigated.

The subsections that follow describe the analytical methods used in this project for each type of compound investigated. 


\subsubsection{Volatile Organic Compound Analysis}

Volatile organic compounds in the iiquid samples were determined using modifications of SY-846 method 5030 and the CLP method for volatiles in water by purge-and-trap gas chromatography-mass spectrometry (GC-MS), which is very similar to SW-846 method 8240 . The main deviation from EPA methodology was that the purge-and-trap step was conducted in a glove box remote from the GC-MS laboratory. Sludge samples were not analyzed for volatile organic compounds.

At the same time that aliquots were taken for purge-and-trap in the glove box, $1.5-\mathrm{mL}$ aliquots were taken in small vials for analysis of ma; : vc.átile organic compounds. This analysis was conducted to "protect" the GC-MS system used for volatile analysis by identifying those samples that were heavily contaminated with higher-boiling compounds (boiling point $\geq 100^{\circ} \mathrm{C}$,. These higher-boiling compounds might be collected in the adsorbent trap and subsequently would contaminate the GC column or overload the GC-MS detector.

This analysis was also used to supplement the purge-and-trap analysis by providing data on highly polar, water-soluble organic compounds that are not determined effectively by the purge-and-trap method (e.g., methyl and ethyl alcohols). Such compounds were suspected because of processes used at ORNL.

\subsubsection{Semivolatile Organic Compound Analysis}

Semivolatile organic compounds were extracted from the liquid and sludge samples after completion of the gross alpha and beta/gamma activity measurements. The gross alpha and beta/gamma measurements had to be taken first to cietermine if the sample could be extracted in a contamination zone hood or if the extraction had to be conducted in a hot cell. The samples were extracted by a modification of SW-846 method 3510 .

Semivolatile organic compounds from the sludge samples were also extracted, using a modification of SW-846 method 3550. Most of the extractions were performed in a hot cell because of the high levels of radioactivity in the sludges, although a few sludges were low enough in radioactivity to allow extraction in a contamination zone hood. Since only limited amounts of sludges were collected, the extracts varied in mass from only 2 to $20 \mathrm{~g}$. The amount of organic material extractable from the sludges for $\mathrm{GC}$ required dilution of the extracts or extraction of smaller masses $(2-5 \mathrm{~g})$. The reporting limits varied from about $500-2500$ to $5000-25,000 \mu \mathrm{g} / \mathrm{kg}$, depending upon the mass extracted and the dilution of the extract.

Two tanks contained organic layers. Samples from these tanks required only dilution and addition of internal standard, per SW-846 method 3580. After testing various dilution ratios with the GC screening method, it was determined that a 100 -fold dilution would keep most of the major constituents detectable by GC within the calibration range of the GC-MS.

All of the extracts were screened using a Megabore capillary column GC with flameionization detection. This screening was conducted to identify those samples requiring dilution to "protect" the GC-MS system and to screen out those samples that contained little organic matter and would not require GC-MS. 
After GC screening, the extracts were measured for total alpha and beta/gamma activity ats then examined for contact and smear alpha and beta/gamma by the Health Physics Inspector. They were than sent to the sample-receiving laboratory for GC-MS analysis. The extracts were analyzed by the CLP GC-MS procedure, which utilizes the method of internal standards.

\subsubsection{Polychlorinated Biphenyl Analysis}

Two tanks contained layers of organic liquids or oils. These layers were analyzed for polychlorinated biphenyls (PCBs) in addition to volatiles and semivolatiles. The preparation method was a modified version of method 3580 .

PCBs were measured by a capillary-column GC method, which is similar to SW-846 method 8080 , except that only the capillary column was used. The samples were injected into a GC device equipped with a $10-\mathrm{m} \times 0.53-\mathrm{mm}$-(interior diameter) $\times 5-\mu \mathrm{m}$ film of bonded SE-30, a fused silica capillary column, and an electron-capture detector. The flow rate of the helium carrier gas was $6 \mathrm{~mL} / \mathrm{min}$, and the total flow rate to the detector was adjusted to 45 $\mathrm{mL} / \mathrm{min}$ with argon/methane $\left(95 / 5\right.$, vol/vol). The column oven was programmed from $140^{\circ}$ to $250^{\circ} \mathrm{C}$ at $8^{\circ} \mathrm{C} / \mathrm{min}$, with a $16-\mathrm{min}$ final isothermal hold, and the injector and detector temperatures were maintained at $250^{\circ}$ and $300^{\circ} \mathrm{C}$ respectively. Quantitation was by the method of internal standards; manually measured peak heights were used for peaks characteristic of each Arochlor mixture. The reporting limit was about 10 to $20 \mathrm{mg} / \mathrm{kg}$.

Data reported were the concentration of each Arochlor and the matrix spike and matrix spike duplicate recoveries.

\subsubsection{Radiochemical and Inorganic Compound Analysis}

The EPA sample preservation procedures that involve maintenance of samples at $4^{\circ} \mathrm{C}$ were avoided for two reasons: (1) the lack of refrigeration facilities for radioactive samples and (2) the possibility of shifts in the equilibrium states of solutions with elevated levels of both $\mathrm{pH}$ and dissolved solids. In addition, on-site filtration upon collection and on-site acid preservation of metals in aqueous samples were not performed because of the danger of handling radioactive materials.

The EPA requirement that states that all samples must initially be run undiluted was not met for samples with high radiation levels or high salt content. Liquid samples were diluted fourfold with nitric acid $(1 \mathrm{~mol})$ prior to analysis to reduce the concentrations of salt and radionuclides to a level that enabled use of existing instrumentation.

Finally, it was not possible to meet the holding time requirements for mercury (26 d) and cyanide (14 d) because of the additional time necessary to analyze radioactive samples. This problem was discussed prior to and during the sampling campaign with both Martin Marietta Energy Systems, Inc., ER peronnel and the EPA Region IV pesonnel and is now being addressed at the national level by the Laboratory Management Division of DOE Headquarters. 
To meet the analytical requirements of this project, a new facility for atomic emission and atomic absorption (AA) spectroscopy was established. This facility included specially adapted instrumentation for inductively coupled plasma (ICP) atomic emission spectroscopy and an AA spectrometer for graphite furnace work and mercury analysis by the cold-vapor technique. Each of these systems was set up and modified for operation in stainless steel enclosures. Both the ICP and the AA spectrometer were equipped with auxiliary exhaust systems to handle the bulk of radioactive aerosols generated during sample analysis. A preoperational readiness review was conducted by the Radioactive Operations Committee on each of these systems prior to their operation with radioactive samples.

The inactive waste tank samples were inspected by a Health Physics representative, and a field survey was performed on each sample before it was packaged and delivered to the HRLAL. On the basis of the field survey results, all liquid samples were handled in radiochemical laboratories (classified as radiation contamination zones). Most of the sludge samples required remote handling for dissolution and dilution before their removal from the hot cell and distribution to various laboratories.

Sample volumes or weights were adjusted to comply with Appendix 7 of the ORNL Health Physics Manual. The limiting factors for laboratory operations were based upon the ${ }^{90} \mathrm{Sr}$ (Class 1; very high radiotoxicity) and ${ }^{137} \mathrm{C}$ (Class 2; high radiotoxicity) content.

Most of the laboratory procedures were simple wet operations, permitting the application of a tenfold modifying factor to the activity levels allowed. Dose rates did not exceed $200-400 \mathrm{mrad} / \mathrm{h}$ at $1.5 \mathrm{in}$. for laboratory operations; the ${ }^{90} \mathrm{Sr}$ activity levels were maintained below $0.1-0.2 \mathrm{mCi}$, and the ${ }^{137} \mathrm{Cs}$ below $1-2 \mathrm{mCi}$. The only problem involved the determination of total and dissolved solids. This required weighing the residue from dried samples. Special care and handling were exercised with the dry samples to prevent dispersing or dusting of the material.

Many of the samples obtained from the inactive waste tanks have high dissolved solids; this phenomenon translates into a high ionic strength. The effects of high ionic strength can have a significant effect on the solution chemistry and various chemical measurements. For example, a potentiometric $\mathrm{pH}$ measurement is actually based upon the hydronium ion activity and not the hydronium activity. The hydronium activity will differ considerably at high ionic strengths. Therefore, the $\mathrm{pH}$ measurements on many of the tank samples can be considered only as apparent $\mathrm{pH}$ values that are not directly related to hydronium ion concentration. The ionic strength will also affect the kinetics of ionic reactions and result in deviations from solubility product behavior. In general, the solution chemistry is far from ideal, and the expected behavior for many species may not be observed.

The physical, inorgar., and radiochemical analyses were conducted at the HRLAL (Building 2026) and the Transuranium Laboratory (Building 7920), both of which serve in the Radioactive Materials Analysis Section, and the Chemical and Physical Analysis Laboratory (Building 4500S), which serves the Inorganic Chemistry Section. The sample receiving, sample distribution, sample preparation, physical measurements, metal analyses, cyanide and sulfide analyses, total organic carbon (TOC), ${ }^{14} \mathrm{C}$ and ${ }^{3} \mathrm{H}$ analyses, and some of the other radiochemical mer surements were performed by $\mathrm{HRLAL}$. The general anion $\left(\mathrm{F}, \mathrm{Cl}, \mathrm{PO}_{4}{ }^{-2}\right.$, $\mathrm{NO}_{3}$, and $\mathrm{SO}_{4}^{-2}$ ) measurements by ion chromatography were performed by the Chemical and 
Physical Analysis Laboratory group. The bulk of the radiochemical data, which include the gross alpha, gross beta, garnma emitters, ${ }^{90} \mathrm{Sr}$, and alpha emitters analyses, were provided by the Transuranium Laboratory.

\subsubsection{Resource Conservation and Recovery Act}

The inactive tanks are categorized as solid waste and are classified as hazardous if the contents of a tank exhibits any of the following specific characteristics: (1) ignitability, (2) reactivity, (3) corrosivity, or (4) toxicity according to an extraction procedure or Extraction Procedure Toxicity (EP-TOX) test. The Toxicity Characteristic Leaching Procedure was not used to determine if the sludge were classified as Resource Conservation and Recovery Act (RCRA) kazardous because EPA had not developed this procedure at the time of the sampling.

The inorganic regulatory characterization for the inactive tanks is separated into classification of the liquid and solid phase for each tank. The following discussion concerning the sludge sample is preliminary because the measurements are based upon the tolc' metal content obtained with a nitric acid leach and not the EP-TOX results. The current available data show only that if a sludge complciely dissolves in the EP-TOX acetate buffer, the data are applicable for the determination of RCRA classification because the measurements would be equivalent to the extraction procedure. Therefore, to arrive at the following classifications, the total metal content has been compared to an EP-TOX equivalent, which accounts for the 20 -fold dilution obtained with the extraction procedure. Only the metals whose total metal content exceeded the EP-TOX equivalent limit were measured in the EP-TOX leachate.

The results of the extraction procedure show that the toxic metals of interest $(\mathrm{Cr}, \mathrm{Pb}$, and $\mathrm{Hg}$ ) are weakly extracted into the $0.05 \mathrm{~mol}$ acetate buffer. Although $\sim 50 \%$ of the solid material from the sludge below dissolved in the buffer solution, the toxic metals are present as insoluble species. Therefore, supported by the EP-TOX results for the inorganic metals which had non-RCRA liquids with an apparent sludge are not RCRA according to the inorganic contents.

In addition, a group from the Analytical Spectroscopy Section located at HRLAL provided surrogate standard and matrix spike data on each sample. Because of the complexity of the chemical composition of the inactive tank samples, the surrogate standard and matrix spike technique was employed as a screening tool to provide qualitative information. This information alerted the analyst to possible elemental and spectral interferences in the variety of different analytical procedures employed.

\subsection{REFERENCES}

1. J. W. Autrey et al., Sampling and Analysis of the Inactive Waste Storage Tank Contents at ORNL, ORNL/ER-13, Office of Environmental, Safety, and Health Compliance, Oak Ridge National Laboratory, Oak Ridge, Tennessee, September 1990.

2. J. W. Autrey et al., Sampling and Analysis of Inactive Waste Tanks TH-2, WC-1, and WC-15, ORNL/ER-19, Office of Environmental, Safety, and Health Compliance, Oak Ridge National Laboratory, Oak Ridge, Tennessee, September 1990. 
3. U.S. Environmental Protection Agency, Test Methods for Evaluating Solid Waste, SW-846, 3rd ed., Office of Solid Waste and Emergency Response, Washington, D.C., November 1986.

4. Oak Ridge National Laboratory, U.S. EPA Contract Laboratory Program, Statement of Work for Inorganic Analysis, SOW No. 787, Oak Ridge, Tennessee, 1987. 


\section{WASTE CHARACTERIZATION SUMMARY FOR THE ORNL CATEGORY D LLLW TANKS}

This section presents the results of the chemical analyses of the Category D (inactive) LLLW tanks. The order in which the tanks are listed corresponds to the priority groups (Groups 1-4) as determined in the risk characterization data manual.

\section{Data reporting conventions}

Some explanation of the reporting conventions is necessary to clarify huw the data are presented. Some data are listed in the tables with letters that indicate .a.portant qualifications. The qualifiers present the following information.

J. The compound was identified by the appropriate retention time and mass spectrum but was observed at a concentration lower than the reporting limit. The reporting limit is the lowest concentration of that compound that can be measured accurately in that sample. The quantitation is therefore an estimate. Only one significant digit is used in the discussion of the results for TIC.

In the discussion that follows, the notation "TIC" means that the compound has been identified solely by its mass spectrum in a machine search of spectra contained in a computer's library. The quantitation is based upon the response factor of the nearest internal standard present in the gas chromatograph. Because of the nature of the quantitation procedure, all values reported are, by definition, estimates and bear the $\mathrm{J}$ qualifier.

E. The concentration of the compound exceeded the calibration range of the instrument; therefore, the result must be considered an estimate.

B. The compound was detected either in the instrumental or sample preparation blank. The blanks usually contained low $\mu \mathrm{g} / \mathrm{L}$ levels of several species, as indicated in one of the later subsections of this section.

BE. The analyte was detected in the sample at concentrations exceeding the usual calibration range, but low levels were also detected in the blank. In almost all cases, the levels observed in the sample considerably exceeded that of the blank.

The absence of data qualifiers indicates that a compound was (1) identified on the basis of retention time and mass spectrum, (2) measured within the calibration range of the instrument, and (3) not found in the associated blank sample.

Some other clarifiers are as follows:

1. The units for suspended solids, total dissolved solids (TSD), total solids, inorganic carbon (IC), and total carbon (TC) are $\mathrm{mg} / \mathrm{mL}$, rather than $\mathrm{mg} / \mathrm{L}$.

2. The units for metals and anions are $\mathrm{mg} / \mathrm{L}$ for aqueous solutions and $\mathrm{mg} / \mathrm{kg}$ for solids. 
3. The units for $\mathrm{CO}_{3}{ }^{2}, \mathrm{HCO}_{3}{ }^{2-}, \mathrm{OH}^{-}$, and $\mathrm{H}^{3+}$ are moles (mol).

4. The units for volatiles and semivolatiles are $\mu \mathrm{g} / \mathrm{L}$ for aqueous solutions and $\mu \mathrm{g} / \mathrm{kg}$ for solids.

5. The detection limits given for physical properties, metals, anions, and alkalinity are those supplied by the laboratory.

6. The detection limits given for the organics are the contract required detection limits (CRQLs) given in the EPA "Statement of Work for Organics," February 1988. These do not represent instrumental detection limits but required reporting limits. Generally, detection limits are not required to be determined for GC/MS and GC analysis. The instrument nust only be able to achieve CRQLs. Therefore, all sample results have been compared to the CRQLs.

7. RCRA limits for metals in soil are derived limits and represent units equivalent to EP-TOX. They are obtained by multiplying the aqueous limit by 20 . If a particular metal failed the total limitation for a sample, the sample was extracted and analyzed for the metal(s) that failed soil characterization.

8. RCRA limits for organics are given in $\mu \mathrm{g} / \mathrm{L}$ sor the sake of clarity. Where no entry is made, no limit exists at this time.

The tank sample table headings refer to sample identification numbers. The " $L$ " prefix indicates a liquid sample; the " $S$ " prefix indicates a sludge sample. In cases where both hard and soft sludge were encountered, the sample is understood to be soft sludge unless the sample prefix is an " $\mathrm{H}$." An "O" prefix on the sample number indicates an organic sample.

Reference is made to the TOC data where such data aid in the interpretation of the organic compound analyses. The surrogate standard and matrix spike recoveries are not discussed in detail in this report because there are no established quality control limits for analysis of these sample matrices by modified EPA methods. 


\subsection{TANK W-5}

\subsubsection{Tank Description}

Tank W-5 is a 170,000-gal storage tank that was built in 1943 for the storage of LLLW. The tank is located in the South Tank Farm in Bethel Valley. The reinforced concrete (Gunite) tank is buried below grade.

\subsubsection{Characterization Summary}

\subsubsection{Aqueous liquid}

The results for Tank W-5 are shown in Tables 2.1.1 and 2.1.2. The major constituents detected by the volatile organic analysis (VOA) were trichloroethenes and tetrachloroethenes at $138 \mu \mathrm{g} / \mathrm{L}$ and $267 \mu \mathrm{g} / \mathrm{L}$ concentrations respectively. Other target analytes were observed, but at much lower levels. The semivolatile organic analysis (SVOA) did not detect any significant material in the liquids. The aqueous liquid sample foamed upon acidification during the preparation for the SVOA.

\subsubsection{Sludge}

Tributylphosphate (TBP) was found as a TIC in the sludge at $8 \mathrm{mg} / \mathrm{kg}$. The SVOA suggested the presence of phthalates. 
Table 21.1. Analytical data for tank W-5

\begin{tabular}{|c|c|c|c|c|c|}
\hline \multirow{2}{*}{ Parameter } & \multirow{2}{*}{$\begin{array}{c}D L \\
(m g / L)\end{array}$} & \multirow{2}{*}{ L73 } & \multirow{2}{*}{ S75 } & \multicolumn{2}{|c|}{ RCRA limit } \\
\hline & & & & $\mathrm{mg} / \mathrm{L}$ & $\mathrm{mg} / \mathrm{k} / \mathrm{s}$ \\
\hline \multicolumn{6}{|c|}{ Phyrical properties and miscellancous data } \\
\hline Density ( $\mathrm{g} / \mathrm{mL})$ & & 1.044 & NA & & \\
\hline Depth (inches) & & 4 & NA & & \\
\hline Suspended solids (mg/mL) & 0.1 & 0.7 & NA & & \\
\hline TDS $(\mathrm{mg} / \mathrm{mL})$ & 0.1 & 47.5 & NA & & \\
\hline TOC $(\mathrm{L}=\mathrm{mg} / \mathrm{L}, \mathrm{S}=\mathrm{mg} / \mathrm{kg})$ & -1.0 & 700 & 4020 & & \\
\hline $\mathrm{TS}(\mathrm{L}=\mathrm{mg} / \mathrm{mL}, \mathrm{S}=\mathrm{mg} / \mathrm{g})$ & 0.1 & 48.2 & 348 & & \\
\hline \multicolumn{6}{|c|}{ RCRA metals $(L=m g / h, S=m g / h g)$} \\
\hline Ag & 0.002 & $<0.002$ & 0.26 & 5.0 & 100 \\
\hline As & 0.40 & $<4$ & $<!$ & 5.0 & 100 \\
\hline $\mathrm{Ba}$ & 0.02 & $<0.2$ & 140 & 100.0 & 2000 \\
\hline $\mathrm{Cd}$ & 0.01 & $<0.08$ & 10 & 1.0 & 20.0 \\
\hline $\mathrm{Cr}$ & 0.10 & 2.7 & $(580)$ & 5.0 & 100 \\
\hline $\mathrm{Hg}$ & 0.005 & 0.41 & 18 & 0.2 & 4.0 \\
\hline $\mathrm{Ni}$ & 0.10 & $<1$ & 52 & NA & NA \\
\hline $\mathrm{Pb}$ & 0.50 & $<2$ & 388 & 5.0 & 100 \\
\hline $\mathrm{Se}$ & 0.05 & $<0.09$ & $(0.81)$ & 1.0 & 20.0 \\
\hline $\mathrm{T}$ & 0.05 & $<0.09$ & $(<0.5)$ & NA & NA \\
\hline \multicolumn{6}{|c|}{ Process metals $(L=m g / L, S=m g / k g)$} \\
\hline $\mathrm{Si}$ & 1.0 & 12.9 & NA & & \\
\hline $\mathrm{U}$ & 0.1 & 296 & 1420 & & \\
\hline \multicolumn{6}{|c|}{ Anions ( $L=m g / L, S=m g / k g)$} \\
\hline $\mathrm{Cl}^{-}$ & 1.0 & 8.6 & $<84$ & & \\
\hline $\mathrm{Cn}$ & 0.24 & $\cdot$ & 0.01 & & \\
\hline$F$ & 1.0 & 56 & 1300 & & \\
\hline $\mathrm{NO}_{3}^{-}$ & 5.0 & 21 & - & & \\
\hline $\mathrm{PO}_{4}^{-2}$ & 5.0 & 420 & $<840$ & & \\
\hline $\mathrm{SO}_{4}^{-2}$ & 5.0 & 26 & $<840$ & & \\
\hline \multicolumn{6}{|c|}{ Alkalinity } \\
\hline $\mathrm{CO}_{3}^{-2}(\mathrm{~mol})$ & 0.01 & 0.16 & - & & \\
\hline $\mathrm{HCO}_{3}^{-}(\mathrm{mol})$ & 0.01 & 0.26 & • & & \\
\hline $\mathrm{pH}$ (units) & 0.1 & 10.6 & NA & & \\
\hline \multicolumn{6}{|c|}{ Radiochamical screening ( $L=B q / m L L, S=B q / g)$} \\
\hline Gross alpha & & $6.0 \mathrm{E}+00$ & $1.3 \mathrm{E}+03$ & & \\
\hline Gross beta & & $4.2 \mathrm{E}+03$ & $1.8 \mathrm{E}+05$ & & \\
\hline \multicolumn{6}{|c|}{ Beta/gamma emitters $(L=B q / m L, S=B q / g$} \\
\hline${ }^{137} \mathrm{Cs}$ & & $5.5 \mathrm{E}+03$ & $3.0 \mathrm{E}+04$ & & \\
\hline${ }^{3} \mathrm{H}$ & & $1.9 \mathrm{E}+00$ & $1.1 \mathrm{E}+01$ & & \\
\hline${ }^{60} \mathrm{Co}$ & & $1.6 \mathrm{E}+02$ & $2.6 \mathrm{E}+02$ & & \\
\hline${ }^{90} \mathrm{Sr}$ & & $9.0 \mathrm{E}+00$ & $2.9 \mathrm{E}+04$ & & \\
\hline \multicolumn{6}{|c|}{ Alpha cmittess $(L=B g$ imL, $S=B q$ tg $)$} \\
\hline${ }^{233} U$ & & $+x^{*}+2$ & $4.6 \mathrm{E}+01$ & & \\
\hline${ }^{238} \mathrm{Pu}$ & & $<2$ & $5.4 \mathrm{E}+01$ & & \\
\hline${ }^{239} \mathrm{Pu}$ & & $<4$ & $6.2 \mathrm{E}+02$ & & \\
\hline${ }^{244} \mathrm{Cm}$ & & $<0.4$ & $3.5 E+02$ & & \\
\hline${ }^{252} \mathrm{Cf}$ & & $<0.2$ & $<4$ & & \\
\hline
\end{tabular}

$\mathrm{D} L=$ Detection limit 
Waste Characterization Data for ORNL Inactive LLLW Tanks Rev. 0 / April 1992

Table 21.2. Organic rnalytical data hits for tank W-5

\begin{tabular}{|c|c|c|c|c|c|}
\hline Parameter & $\begin{array}{l}\text { CRQL } \\
(\mu \mathrm{g} / \mathrm{L})\end{array}$ & L73 & S75 & $\begin{array}{l}\text { RCRA unit } \\
(\mu \mathrm{g} / \mathrm{L})\end{array}$ & $\begin{array}{c}\text { CRQL soil } \\
(\mu \mathrm{g} / \mathrm{kg})\end{array}$ \\
\hline \multicolumn{6}{|c|}{ Volatile ongunic compound anabusis ( $L=\mu g / L, S=\mu g / \mathrm{kg})$} \\
\hline Acetone & 10 & $19 \mathrm{~B}$ & & & \\
\hline Carbon tetrachloride & 5 & $68 \mathrm{~B}$ & & 500 & \\
\hline Chloroform & 5 & 6 & & 6,000 & \\
\hline Methylene chloride & 5 & $6 \mathrm{~B}$ & & & \\
\hline Tetrachloroethene & 5 & $267 \mathrm{~B}$ & & 700 & \\
\hline Trichloroethene & 5 & 138 & & 500 & \\
\hline \multicolumn{6}{|c|}{ Semivolatile compound an ahwis $(L=\mu g / L, S=n g / \mathrm{kg})$} \\
\hline Bis(2-ethylhexyl)phthalate & & & 8,000 & & 330 \\
\hline Di-n-butylphthalate & $10\left(\mathrm{H}_{2} \mathrm{O}\right)$ & $18 \mathrm{~J}$ & $350 \mathrm{~J}$ & & 330 \\
\hline Diethylphthalate & & & $230 \mathrm{~J}$ & & 339 \\
\hline Fluoranthene & & & $230 \mathrm{~J}$ & & 330 \\
\hline Phenanthrene & & & $440 \mathrm{~J}$ & & 330 \\
\hline Pyrene & & & $230 \mathrm{~J}$ & & 330 \\
\hline
\end{tabular}

$\mathrm{CRQL}=$ contract required quantitation limit 


\subsection{TANK W-6}

\subsubsection{Tank Description}

Tank W-6 is a 170,000 -gal Gunite tank located in the Bethel Valley South Tank Farm. The underground tank was constructed in 1943 for the storage of LLLW.

\subsubsection{Characterization Summary}

The results for this tank are shown in Tables 2.2 .1 and 2.2.2.

\subsubsection{Aqueous liquid}

The principal volatile organics were trichloroethene and tetrachloroethenes. The SVOA of the aqueous liquids showed only benzoic acid at $300 \mu / \mathrm{L}$ in $\mathrm{L} 79$. Sample $\mathrm{L} 78$ turned green upon the addition of the SVOA matrix spike, but the surrogate recoveries were acceptable.

\subsubsection{Sludge}

A few polycyclic aromatic hydrocarbons (PAHs) and phthalates were determined in the s!!udge. 
Waste Characterization Data for ORNL Inactive LLLW Tanks

Table 2.2.1. Analytical data for tank W-6

\begin{tabular}{|c|c|c|c|c|c|}
\hline Parameter & $\begin{array}{c}D L \\
(m g /)\end{array}$ & $\llcorner 77$ & 1.78 & L79 & S80 \\
\hline \multicolumn{6}{|c|}{ Physical properties and miscellaneous data } \\
\hline Density $(\mathrm{g} / \mathrm{mL})$ & & 0.999 & 1.009 & 1.059 & NA \\
\hline Depth (inches) & & 4 & 30 & 54 & NA \\
\hline Suspended solids ( $\mathrm{mg} / \mathrm{mL})$ & 0.1 & $<0.1$ & $<0.1$ & 0.1 & NA \\
\hline $\operatorname{TDS}(\mathrm{mg} / \mathrm{mL})$ & 0.1 & 2.5 & 14.8 & 74.0 & NA \\
\hline $\operatorname{TOC}(\mathrm{L}=\mathrm{mg} / \mathrm{L}, \mathrm{S}=\mathrm{mg} / \mathrm{kg})$ & 1.0 & 2.0 & 2.0 & 180 & 9110 \\
\hline TS $(L=m g / m L, S=m g / g)$ & 0.1 & 2.5 & 14.7 & 74.1 & 406 \\
\hline \multicolumn{6}{|c|}{$R C R A$ mesals $(L=m g / L, S=m g / k g)$} \\
\hline $\mathrm{Ag}$ & 0.002 & $<0.002$ & $<0.002$ & $<0.002$ & 0.006 \\
\hline As & 0.40 & $<4$ & $<4$ & $<4$ & $(<2)$ \\
\hline $\mathrm{Ba}$ & 0.02 & $<0.2$ & $<0.2$ & $<0.2$ & $(350)$ \\
\hline Cd & 0.01 & $<0.08$ & $<0.08$ & $<0.08$ & $<1$ \\
\hline $\mathrm{Cr}$ & 0.10 & $<0.3$ & 2.6 & 32 & $(2400)$ \\
\hline $\mathrm{Hg}$ & 0.005 & $<0.01$ & $<0.01$ & 0.07 & 36 \\
\hline $\mathrm{Ni}$ & 0.10 & $<1$ & $<1$ & 1.5 & 85 \\
\hline $\mathrm{Pb}$ & 0.50 & $<2$ & $<2$ & $<2$ & 1100 \\
\hline $\mathrm{Se}$ & 0.05 & $<0.09$ & $<0.09$ & $<0.09$ & $(<2)$ \\
\hline $\mathrm{T}$ & 0.05 & $<0.09$ & $<0.09$ & $<0.09$ & $<2$ \\
\hline \multicolumn{6}{|c|}{ Process metals $(L=m g / h, S=m g / k g)$} \\
\hline $\mathrm{Si}$ & 1.0 & 3.99 & 2.98 & 1.65 & NA \\
\hline $\mathrm{U}$ & 0.1 & 16.2 & 52.3 & 69.8 & 6340 \\
\hline \multicolumn{6}{|c|}{ Anions $(L=m g / L, S=m g / k g)$} \\
\hline $\mathrm{Cl}^{-}$ & 1.0 & 1.3 & 4.5 & 12.0 & $<800$ \\
\hline $\mathrm{Cn}$ & 0.01 & $<0.07$ & 0.05 & 0.41 & * \\
\hline $\mathrm{F}^{-}$ & 1.0 & 5.1 & 27 & 49 & 4000 \\
\hline $\mathrm{NO}_{3}$ & 5.0 & 73 & 250 & 710 & • \\
\hline $\mathrm{PO}_{4}^{-2}$ & 5.0 & $<5$ & 31 & 210 & $<2500$ \\
\hline $\mathrm{SO}_{4}^{-2}$ & 5.0 & 4.8 & 34 & 330 & $<2500$ \\
\hline \multicolumn{6}{|c|}{ Alkaliniry } \\
\hline $\mathrm{CO}_{3}^{-2}(\mathrm{~mol})$ & 0.01 & $<0.01$ & 0.02 & 0.18 & * \\
\hline $\mathrm{HCO}_{3}^{\circ}(\mathrm{mol})$ & 0.01 & 0.02 & 0.11 & 0.10 & - \\
\hline pH (units) & 0.1 & 8.7 & 10.4 & 11.8 & NA \\
\hline \multicolumn{6}{|c|}{ Radiochemical screening ( $L=B q / m I, S=B q / g)$} \\
\hline Gross alpha & & $1.0 \mathrm{E}+00$ & $3.0 \mathrm{E}+00$ & $3.0 \mathrm{E}+00$ & $8.0 \mathrm{E}+03$ \\
\hline Gross beta & & $2.8 \mathrm{E}+0.3$ & $6.0 \mathrm{E}+03$ & $1.9 \mathrm{E}+04$ & $1.0 \mathrm{E}+06$ \\
\hline \multicolumn{6}{|c|}{ Beta/gamma cmitters $(L=B q / m L, S=B q / g)$} \\
\hline${ }^{137} \mathrm{Cs}$ & & $1.6 \mathrm{E}+03$ & $5.6 \mathrm{E}+03$ & $2.0 \mathrm{E}+04$ & $8.2 \mathrm{E}+04$ \\
\hline${ }^{3} \mathrm{H}$ & & $6.3 E-01$ & $1.4 \mathrm{E}+00$ & $3.2 \mathrm{E}+00$ & $9.2 \mathrm{E}+00$ \\
\hline$\omega_{\mathrm{CO}}$ & & $1.3 \mathrm{E}+01$ & $2.8 \mathrm{E}+01$ & $9.0 \mathrm{E}+01$ & $9.1 \mathrm{E}+02$ \\
\hline${ }^{90} \mathrm{Sr}$ & & $3.1 \mathrm{E}+02$ & $7.8 \mathrm{E}+01$ & $1.9 \mathrm{E}+02$ & $3.6 \mathrm{E}+05$ \\
\hline \multicolumn{6}{|c|}{ Alpha anitters $(L=B q / m L, S=B q / g)$} \\
\hline${ }^{233} \mathrm{U}$ & & $*$ & 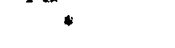 & $\cdot$ & $2.7 \mathrm{E}+02$ \\
\hline${ }^{238} \mathrm{Pu}$ & & $<2$ & $<2$ & $<2$ & $2.1 \mathrm{E}+02$ \\
\hline${ }^{239} \mathrm{Pu}$ & & $<4$ & $<4$ & $<4$ & $1.8 \mathrm{E}+03$ \\
\hline${ }^{244} \mathrm{Cm}$ & & $<0.4$ & $<0.4$ & $<0.4$ & 4.2.E +03 \\
\hline${ }^{252} \mathrm{Cl}$ & & $<0.2$ & $<0.2$ & $<0.2$ & $<8$ \\
\hline
\end{tabular}

DL $=$ Detection limit 
Waste Characterization Data for ORNL Inactive LLLW Tanks Rev. 0 / June 1992.

Table 222 Organic ansynical date bits for tant W.6

\begin{tabular}{|c|c|c|c|c|c|c|c|}
\hline Parameter & $\begin{array}{l}\text { CRQL } \\
(\mu \mathrm{g} /)\end{array}$ & L.77 & L78 & L.79 & $\mathbf{s} 080$ & $\begin{array}{c}\text { RCRA } \\
\text { limil }\end{array}$ & $\begin{array}{l}\text { CRQL } \\
(4 \& / \times B)\end{array}$ \\
\hline \multicolumn{8}{|c|}{ 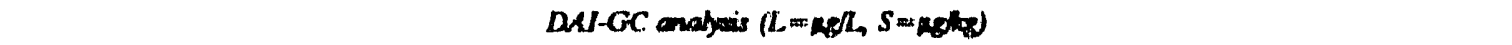 } \\
\hline 1.Propyl alcohol & & & & 3,000 & & & \\
\hline \multicolumn{8}{|c|}{ 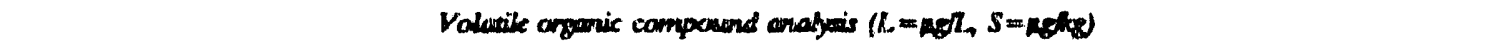 } \\
\hline 2-Butanone & 10 & & & 75 & & 200,000 & \\
\hline 2-Hexanone & 10 & & & 9 & & & \\
\hline 4-Methyt-2-pentanone: & 10 & & $9 \mathrm{~J}$ & 91 & & & \\
\hline Acetone & 10 & $67 \mathrm{~B}$ & $44 \mathrm{~B}$ & $111 \mathrm{~B}$ & & & \\
\hline Bemaene & 5 & & $2 \mathrm{~J}$ & 10 & & 500 & \\
\hline Curbon tetrachloride & 5 & $4 \mathrm{JB}$ & & & & 500 & \\
\hline Chloroform & 5 & $5 \mathrm{~J}$ & 9 & $3 \mathrm{~J}$ & & 6,000 & \\
\hline Methylene chloride & 5 & $9 \mathrm{~B}$ & $8 \mathrm{~B}$ & $7 \mathrm{~B}$ & & & \\
\hline Tetrachlorvethene & 5 & $47 \mathrm{~B}$ & $158 \mathrm{~B}$ & $507 \mathrm{BE}$ & & 700 & \\
\hline Toluene & 5 & $6 \mathrm{~B}$ & $10 \mathrm{~B}$ & $10 \mathrm{~B}$ & & & \\
\hline Trichloroethene & 5 & 13 & 24 & 82 & & 500 & \\
\hline \multicolumn{8}{|c|}{ 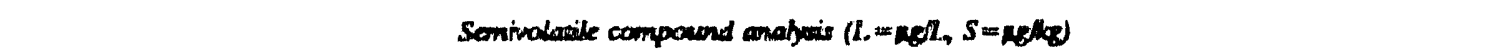 } \\
\hline Bentroic acid & 50 & & & $290 \mathrm{~J}$ & & & \\
\hline Bis(2-elhylhexyl)phithalate & 10 & & & & 31,000 & & 1,600 \\
\hline Di-n-butylphthalste & 10 & & & & $210 \mathrm{~J}$ & & 330 \\
\hline Diethylphthalate & 10 & & & & $150 \mathrm{~J}$ & & 330 \\
\hline Muoranthene & 10 & & & & $340 \mathrm{~J}$ & & 330 \\
\hline Yhenanthrene & 10 & & & & $680 \mathrm{~J}$ & & 330 \\
\hline Pyrene & 10 & & & & $350 \mathrm{~J}$ & & 330 \\
\hline
\end{tabular}

$\overline{C R Q L}=$ contract required quantitation limit 


\section{TANK W-7}

\subsection{Tank Description}

Tank W-7 is a 170,000-gal Gunite tank located in the Bethel Valley South Tank Farm. The underground tank was constructed in 1943 for the storage of LLLW.

\subsection{Characterization Summary}

The results for this tank are shown in Tables 2.3.1 and 2.3.2.

\subsubsection{Aqueous liquid}

The major organic constituents were $14 \mathrm{mg} / \mathrm{L}$ of methanol and $\sim 1000 \mu \mathrm{g} / \mathrm{L}$ of carbon tetrachloride in L82. The data for the sample identification numbers with A and B suffixes are for duplicate determinations. These data show very good agreement, considering the low concentrations and two purging steps in the analysis. Benzoic acid $(2000 \mu \mathrm{g} / \mathrm{L})$ was the only TCL compound detected. Dibromonitrophenol was estimated at $700 \mu \mathrm{g} / \mathrm{L}$ as a TIC.

\subsubsection{Sludge}

Fluoranthene and several phthalates were detected in the sludges. Four of the five SVOA surrogate standard recoveries (particularly for the acid surrogate standards) from the soft sludge, S84, were very low, suggesting problems with the extraction step. Surrogate standard recoveries were somewhat better for the hard sludge, H85. This difference in recovery for the surrogates between soft and hard sludges was observed in other samples.

The samples for tanks W-8 and W-9, except for the sludges, were prepared together. 
Waste Characterization Data for ORNL Inactive LLL.W Tanks Rev. 0/ June 1992

Table 23.1. Analytical data for tant W-7

\begin{tabular}{|c|c|c|c|c|c|c|}
\hline \multirow{2}{*}{ Parameter } & \multirow{2}{*}{$\begin{array}{c}D L \\
(\mathrm{mg} / \mathrm{L})\end{array}$} & \multirow{2}{*}{ H85 } & \multirow{2}{*}{182} & \multirow{2}{*}{$\$ 84$} & \multicolumn{2}{|c|}{ RCRA limit } \\
\hline & & & & & $\mathrm{mg} / \mathrm{L}$ & $\mathrm{mg} / \mathrm{kg}$ \\
\hline \multicolumn{7}{|c|}{ Phosical properties and miscellaneous data } \\
\hline Density ( $g / m L)$ & & NA & 1.130 & NA & & \\
\hline Depth (inches) & & NA & 3 & NA & & \\
\hline Suspended solids (mg/mL) & 0.1 & NA & 11.3 & NA & & \\
\hline $\operatorname{TDS}(\mathrm{mg} / \mathrm{mL})$ & 0.1 & NA & 158.7 & NA & & \\
\hline $\operatorname{TOC}(L=m g / L, S=m g / k g)$ & 1.0 & 4,010 & 50.0 & 9,030 & & \\
\hline $\mathrm{TS}(\mathrm{L}=\mathrm{mg} / \mathrm{mL}, \mathrm{S}=\mathrm{mg} / \mathrm{g})$ & 0.1 & 360 & 170 & 367 & & \\
\hline \multicolumn{7}{|c|}{$R C R A$ merals $(L=m g / L, s=m g / k g)$} \\
\hline $\mathrm{Ag}$ & 0.002 & 0.12 & $\cdot-1$ & 0.36 & 5.0 & 100 \\
\hline As & 0.40 & $<6$ & $<4$ & $<6$ & 5.0 & 100 \\
\hline $\mathrm{Ba}$ & 0.02 & (16) & $<0.2$ & $(1,300)$ & 10.0 & 2,000 \\
\hline Cd & 0.01 & 2.2 & $<0.08$ & 2.0 & 1.0 & 20.0 \\
\hline $\mathrm{Cr}$ & 0.10 & $(130)$ & 145 & $(660)$ & 5.0 & 100 \\
\hline $\mathrm{Hg}$ & 0.005 & 52 & (11) & 141 & 0.2 & 4.0 \\
\hline $\mathrm{Ni}$ & 0.10 & 22 & $<1$ & 84 & NA & NA \\
\hline $\mathrm{Pb}$ & 0.50 & $(77)$ & $<2$ & $(300)$ & 5.0 & 100 \\
\hline Se & 0.05 & $(<1)$ & $<2$ & $(<1)$ & 1.0 & 20.0 \\
\hline $\mathrm{T} 1$ & 0.05 & $<1$ & $<2$ & $<1$ & & \\
\hline \multicolumn{7}{|c|}{ Process mats $(L=m g / L, S=m g / h g)$} \\
\hline $\mathrm{Si}$ & 1.0 & NA & 7.35 & NA & & \\
\hline 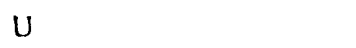 & 0.1 & 86,000 & 8,530 & 45,000 & & \\
\hline \multicolumn{7}{|c|}{ Anions $(L=m g / h, s=m g / g)$} \\
\hline $\mathrm{Cl}$ - & 1 & $\cdot$ & 29 & $<1.2 \%$ & & \\
\hline $\mathrm{Cn}$ & 0.01 & - & $<0.06$ & $\bullet$ & & \\
\hline$F$ & 1 & • & 11 & 1,000 & & \\
\hline $\mathrm{NO}_{3}$ & 5 & - & 440 & • & & \\
\hline $\mathrm{PO}_{4}^{-2}$ & 5 & - & 29 & - & & \\
\hline $\mathrm{SO}_{4}^{-2}$ & 5 & - & 55 & $6.7 \%$ & & \\
\hline \multicolumn{7}{|c|}{ Alkalininy } \\
\hline $\mathrm{CO}_{3}^{-2}(\mathrm{~mol})$ & 0.01 & - & 0.21 & - & & \\
\hline $\mathrm{HCO}_{3} \cdot(\mathrm{mol})$ & 0.01 & - & 0.33 & $\cdot$ & & \\
\hline $\mathrm{pH}$ (units) & 0.1 & NA & 10.7 & NA & & \\
\hline \multicolumn{7}{|c|}{ Radiochemical screaning ( $L=B q\left(m l^{\prime}, S=B q / g\right)$} \\
\hline Gross alpha & & $8.0 \mathrm{E}+03$ & $2.0 \mathrm{E}+02$ & $1.4 \mathrm{E}+04$ & & \\
\hline Gross beta & & $1.7 E+06$ & $5.6 \mathrm{E}+05$ & $3.1 \mathrm{E}+06$ & & \\
\hline \multicolumn{7}{|c|}{ Belatgamme emisters $\left(L=B \mathrm{~g} / \mathrm{mL}_{-}, S=\mathrm{Bg} / \mathrm{g}\right)$} \\
\hline${ }^{134} \mathrm{Cs}$ & & $*$ & - & $1.0 \mathrm{E}+02$ & & \\
\hline${ }^{137} \mathrm{Cs}$ & & $1.0 \mathrm{E}+06$ & $6.4 E+0.5$ & $1.2 E+06$ & & \\
\hline${ }^{152} \mathrm{Eu}$ & & $\cdot$ & - & $1.6 \mathrm{E}+03$ & & \\
\hline${ }^{154} \mathrm{Eu}$ & & $4.5 E+02$ & $\cdot$ & $1.3 \mathrm{E}+03$ & & \\
\hline${ }^{3} \mathrm{H}$ & & $8.3 E+01$ & $1.9 \mathrm{E}+01$ & $1.2 \mathrm{E}+02$ & & \\
\hline${ }^{60} \mathrm{Co}$ & & $2.0 \mathrm{E}+03$ & $2.9 E+02$ & $5.0 \mathrm{E}+03$ & & \\
\hline $4 \mathrm{Sr}$ & & $3.1 E+0.5$ & $6.0 \mathrm{E}+02$ & $8.1 E+05$ & & \\
\hline
\end{tabular}


Waste Characterization Data for ORNL Inactive LLLW Tanks Rev. 0/June 1992

Table 23.1 (continued)

\begin{tabular}{|c|c|c|c|c|c|c|}
\hline \multirow{2}{*}{ Parameter } & \multirow{2}{*}{$\underset{(\mathrm{mg} / \mathrm{L})}{\mathrm{DL}}$} & \multirow{2}{*}{$\mathrm{H} 85$} & \multirow{2}{*}{ L82 } & \multirow{2}{*}{ S84 } & \multicolumn{2}{|c|}{ RCRA limit } \\
\hline & & & & & $\mathrm{mg} / \mathrm{L}$ & $\mathrm{mg} / \mathrm{kg}$ \\
\hline \multicolumn{7}{|c|}{ Alpha enituers ( $L=B q / m L, S=B q / g)$} \\
\hline${ }^{233} \mathrm{U}$ & & $1.7 E+03$ & - & $5.4 \mathrm{E}+02$ & & \\
\hline${ }^{228} \mathrm{Pu}$ & & $6.4 \mathrm{E}+02$ & $<2$ & $1.1 \mathrm{E}+03$ & & \\
\hline${ }^{238} \mathrm{Th} /{ }^{232} \mathrm{Th}$ & & - & $8.5 E+01$ & - & & \\
\hline $238 \mathrm{U}$ & & $1.6 \mathrm{E}+03$ & - & $\cdot$ & & \\
\hline${ }^{239} \mathrm{Pu}$ & & $4.8 \mathrm{E}+02$ & $<4$ & $2.4 \mathrm{E}+03$ & & \\
\hline${ }^{241} \mathrm{Am}$ & & $\cdot$ & - & $5.7 \mathrm{E}+02$ & & \\
\hline${ }^{244} \mathrm{Cm}$ & & $2.5 E+03$ & $<0.4$ & $4.2 E+03$ & & \\
\hline${ }^{252} \mathrm{Cf}$ & & $<4$ & $<0.2$ & $<14$ & & \\
\hline
\end{tabular}

$\mathrm{D} \mathrm{L}=$ Detection limit

Table 23.2. Organic analytical data hits for tank W-7

\begin{tabular}{|c|c|c|c|c|c|c|c|}
\hline \multirow{2}{*}{ Parameter } & \multirow{2}{*}{$\begin{array}{l}\text { CRQL } \\
(\mu \mathrm{g} / \mathrm{L})\end{array}$} & \multirow{2}{*}{$\mathrm{H} 85$} & \multirow{2}{*}{1.82} & \multirow{2}{*}{ L82B } & \multirow{2}{*}{ S84 } & \multicolumn{2}{|c|}{ RCRA limit } \\
\hline & & & & & & $\mu g / L$ & $\mu \mathrm{g} / \mathrm{kg}$ \\
\hline \multicolumn{8}{|c|}{ 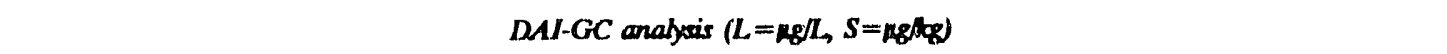 } \\
\hline Methy! alcohol & & & 14,000 & & & & \\
\hline \multicolumn{8}{|c|}{ Volatile arganic compound anabsis $(L=m g / L, S=$ ngikg) } \\
\hline 4-Methyl-2-pentanone & 10 & & 7 & 7 & & & \\
\hline Acetone & 10 & & $26 \mathrm{~B}$ & $31 \mathrm{~B}$ & & & \\
\hline Carbon tetrachloride & 10 & & $1,088 \mathrm{BE}$ & $1,021 \mathrm{BE}$ & & 500 & \\
\hline Chloroform & 5 & & $3 \mathrm{~J}$ & $3 \mathrm{~J}$ & & 6,000 & \\
\hline Methylene chloride & 5 & & $7 \mathrm{~B}$ & $4 \mathrm{~B}$ & & & \\
\hline Tetrachloroethene & 5 & & $24 \mathrm{~B}$ & $39 \mathrm{~B}$ & & 700 & \\
\hline Toluene & 5 & & $14 \mathrm{~B}$ & $9 \mathrm{~B}$ & & & \\
\hline \multicolumn{8}{|c|}{ 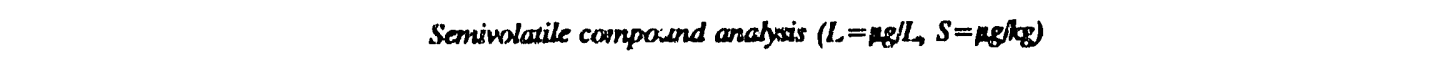 } \\
\hline Benzoic acid & 50 & & $1,900 \mathrm{~J}$ & & & & 1,600 \\
\hline Bis(2-ethylhexyl)phthalate & 10 & & & & 57,0000 & & 330 \\
\hline Di-n-butylphthalate & 10 & $2,200 \mathrm{~J}$ & & & $300 \mathrm{~J}$ & & 330 \\
\hline Diethyiphthalate & 10 & $510 \mathrm{~J}$ & & & $200 \mathrm{~J}$ & & 330 \\
\hline Fluoranthene & 10 & & & & $260 \mathrm{~J}$ & & 330 \\
\hline
\end{tabular}

$\mathrm{CRQL}=$ contract required quantitation limit 


\section{TANK W-8}

\subsection{Tank Description}

Tank W-8 is a 170,000 -gal Gunite tank located in the Bethel Valley South Tank Farm. The underground tank was constructed in 1943 for the storage of LLLW.

\subsection{Characterization Summary}

Tables 2.4.1 and 2.4.2 list the findings for this tank.

\subsubsection{Aqueous liquid}

The concentrations of both volatile and semivolatile organic compounds were very low.

The main SVOA TICs were dibromocyclohexane ( $300 \mu \mathrm{g} / \mathrm{L}$ in $\mathrm{L} 86$ alone), dibromonitrophenol (500 and $700 \mu \mathrm{g} / \mathrm{L}$ in L86 and L87, respectively), and TBP (20 mg/L in each liquid). The results suggest bromination reactions may have occurred.

Only the sludge was found to contain significant detectable organic compounds, which were mostly PAHs. The TICs were mostly unidentified hydrocarbons and TBP (700 mg/kg).

The acidic surrogate standard compounds were very poorly recovered from the aqueous liquids, suggesting that acidic compounds, if present in the samples, may not have been extracted or detected. The two chlorinated phenolic matrix spike compounds also were poorly recovered. 
Table 24.1. Analytical data for tank W-8

\begin{tabular}{|c|c|c|c|c|}
\hline Parameter & $\begin{array}{c}D L \\
(\mathrm{mg} / \mathrm{L})\end{array}$ & L86 & L87 & S88 \\
\hline \multicolumn{5}{|c|}{ Physical properties and miscellaneous data } \\
\hline Density (g/mL) & & 1.020 & 1.027 & NA \\
\hline Depth (inches) & & 4 & 16 & NA \\
\hline Suspended solids $(\mathrm{mg} / \mathrm{mL})$ & 0.1 & $<0.1$ & $<0.1$ & NA \\
\hline TDS (mg/mL) & 0.1 & 29.4 & 37.8 & NA \\
\hline $\mathrm{TOC}(\mathrm{L}=\mathrm{mg} / \mathrm{L}, \mathrm{S}=\mathrm{mg} / \mathrm{kg})$ & 1.0 & 720 & 1,460 & 11,300 \\
\hline $\mathrm{TS}(\mathrm{L}=\mathrm{mg} / \mathrm{mL}, \mathrm{S}=\mathrm{mg} / \mathrm{g})$ & 0.1 & 29.4 & 37.6 & 558 \\
\hline \multicolumn{5}{|c|}{$R C R A$ metals $(L=m g / L, S==m g / k g)$} \\
\hline Ag & 0.002 & 0.007 & $<0.002$ & 1.3 \\
\hline As & 0.4 & $<4$ & $<4$ & $<4$ \\
\hline $\mathrm{Ba}$ & 0.02 & $<0.2$ & $<0.2$ & $(38)$ \\
\hline $\mathrm{Cd}$ & 0.01 & $<0.2$ & $<0.2$ & 4.1 \\
\hline $\mathrm{Cr}$ & 0.1 & 12. & 18 & $(410)$ \\
\hline $\mathrm{Hg}$ & 0.005 & 0.09 & 0.17 & 50 \\
\hline $\mathrm{Ni}$ & 0.10 & $<1$ & $<1$ & 160 \\
\hline $\mathrm{Pb}$ & 0.5 & $<2$ & $<2$ & $(1,800)$ \\
\hline $\mathrm{Se}$ & 0.05 & $<0.09$ & $<0.09$ & $(<0.7)$ \\
\hline $\mathrm{T}$ & 0.05 & $<0.09$ & $<0.09$ & $<0.7$ \\
\hline \multicolumn{5}{|c|}{ Process mesals ( $L=m g / L, S=m g / k g)$} \\
\hline $\mathrm{Si}$ & 1.0 & 1.39 & 1.14 & NA \\
\hline U & 0.1 & 817 & 1,760 & 8,560 \\
\hline \multicolumn{5}{|c|}{ Anions $(L=m g / L, S=m g / h g)$} \\
\hline $\mathrm{Cl}^{\circ}$ & 1 & 290 & 840 & $<100$ \\
\hline $\mathrm{Cn}$ & 0.01 & 0.33 & 0.58 & $\cdot$ \\
\hline $\mathrm{F}$ & 1 & $<50$ & $<125$ & $<100$ \\
\hline $\mathrm{NO}_{3}$ & 5 & 2,700 & 7,300 & - \\
\hline $\mathrm{PO}_{4}^{-2}$ & 5 & $<250$ & $<625$ & $<500$ \\
\hline $\mathrm{SO}_{4}^{-2}$ & 5 & 1,400 & 4,900 & $<500$ \\
\hline \multicolumn{5}{|c|}{ Alkalinity } \\
\hline $\mathrm{CO}_{3}^{-2}(\mathrm{~mol})$ & 0.01 & 0.01 & 0.02 & * \\
\hline $\mathrm{HCO}_{3}(\mathrm{~mol})$ & 0.01 & 0.04 & 0.07 & - \\
\hline pH (units) & 0.1 & 9.6 & 9.7 & NA \\
\hline \multicolumn{5}{|c|}{ Radiochernical screening ( $L=B$ q $/ m L, S=B$ glg) } \\
\hline Gross alpha & & $1.5 \mathrm{E}+02$ & $2.0 \mathrm{E}+02$ & $2.9 \mathrm{E}+04$ \\
\hline Gross beta & & $2.4 \mathrm{E}+05$ & $2.8 \mathrm{E}+0.5$ & $6.7 \mathrm{E}+06$ \\
\hline \multicolumn{5}{|c|}{ Beculganuna anitters $(L=B q / m L, S=B q / 8)$} \\
\hline${ }^{137} \mathrm{Cs}$ & & $3.4 E+05$ & $4.0 \mathrm{E}+05$ & $4.7 \mathrm{E}+04$ \\
\hline${ }^{152} \mathrm{Eu}$ & & $\cdot$ & $\cdot$ & $9.0 \mathrm{E}+01$ \\
\hline${ }^{154} \mathrm{Eu}$ & & $\cdot$ & $\cdot$ & $1.4 \mathrm{E}+02$ \\
\hline${ }^{3} \mathrm{H}$ & & $8.0 \mathrm{E}+00$ & $8.8 E+00$ & $0.0 \mathrm{E}+00$ \\
\hline${ }^{60} \mathrm{Co}$ & & $3.0 \mathrm{E}+02$ & $5.9 \mathrm{E}+02$ & $4.0 \mathrm{E}+02$ \\
\hline${ }^{90} \mathrm{Sr}$ & & $6.6 \mathrm{E}+02$ & $4.6 \mathrm{E}+02$ & $2.8 \mathrm{E}+06$ \\
\hline
\end{tabular}


Waste Characterization Data for ORNL Inactive LLLW Tanks Rev. 0 / June 1992

Table 23.1 (continued)

\begin{tabular}{|c|c|c|c|c|}
\hline Parameter & $\begin{array}{c}\mathrm{D} \mathrm{L} \\
(\mathrm{mg} / \mathrm{L})\end{array}$ & L86 & L87 & S88 \\
\hline \multicolumn{5}{|c|}{ Alpha emitters $(L=B q / m L, S=B q / g)$} \\
\hline${ }^{233} \mathrm{U}$ & & $<2$ & $<2$ & $1.6 \mathrm{E}+03$ \\
\hline${ }^{238} \mathrm{Pu}$ & & $<2$ & $<2$ & $2.9 E+03$ \\
\hline${ }^{239} \mathrm{Pu}$ & & $<2$ & $<2$ & $3.2 \mathrm{E}+03$ \\
\hline${ }^{241} \mathrm{Amn}$ & & • & • & $2.0 \mathrm{E}+02$ \\
\hline${ }^{244} \mathrm{Cm}$ & & $<2$ & $<2$ & $1.5 \mathrm{E}+04$ \\
\hline${ }^{252} \mathrm{Cf}$ & & $<2$ & $<2$ & $<4$ \\
\hline
\end{tabular}

$\mathrm{DL}=$ Detection limit

Table 24.2. Onganic anatytical data hits for tant W-8

\begin{tabular}{|c|c|c|c|c|c|c|}
\hline Parameter & $\begin{array}{l}\text { CRQL } \\
(\mu \mathrm{g} / \mathrm{L})\end{array}$ & L86 & L87 & S88 & $\begin{array}{c}\text { RCRA limit } \\
(\mu \mathrm{g} / \mathrm{L})\end{array}$ & $\begin{array}{l}\text { CRQL } \\
(\mu \mathrm{g} / \mathbf{k g})\end{array}$ \\
\hline \multicolumn{7}{|c|}{$D A I-G C$ analysis $(L=\mu g /, S=\mu g / \mathrm{kg})$} \\
\hline Acetone & & 1,000 & 3,000 & & & \\
\hline Methyl alcohol & & & 1,000 & & & \\
\hline Methyl ethyl ketone & & & 1,000 & & & \\
\hline N-Butyl alcohol & & & 2,000 & & & \\
\hline \multicolumn{7}{|c|}{ Volatile organic compound anabuis ( $L=\mu g / L, S=n g / \mathrm{kg})$} \\
\hline 2-Hexanone & 10 & 41 & & & & \\
\hline 4-Methyl-2-pentanone & 10 & 15 & & & & \\
\hline Acetone & 10 & $64 \mathrm{~B}$ & $45 \mathrm{~B}$ & & & \\
\hline Benzene & 5 & $3 \mathrm{~J}$ & 6 & & 500 & \\
\hline Methylene chloride & 5 & $12 \mathrm{~B}$ & $7 \mathrm{~B}$ & & & \\
\hline Toluene & 5 & $3 \mathrm{BJ}$ & $4 \mathrm{BJ}$ & & & \\
\hline \multicolumn{7}{|c|}{ Senivolatile compound analysis $(L=\mu g /, S S=\mu g / k g)$} \\
\hline 2-Methylnaphthalene & 10 & & & $1,300 \mathrm{~J}$ & & 330 \\
\hline Di-n-butylphshalate & 10 & & $17 \mathrm{~J}$ & $630 \mathrm{~J}$ & & 330 \\
\hline Diethylphthalate & 10 & & & $430 \mathrm{~J}$ & & 330 \\
\hline Fluoranthene & 10 & & & $740 \mathrm{~J}$ & & 330 \\
\hline Naphthalene & 10 & & $28 \mathrm{~J}$ & $2,600 \mathrm{~J}$ & & 330 \\
\hline Phenanthrene & 10 & & & $1,300 \mathrm{~J}$ & & 330 \\
\hline Pyrene & 10 & & & $850 \mathrm{~J}$ & & 330 \\
\hline
\end{tabular}

$\mathrm{CRQL}=$ contract required quantitation limit 


\subsection{TANK W-9}

\subsubsection{Tank Description}

Tank W-9 is a 170,000-gal Gunite tank located in the Bethel Valley South Tank Farm. The underground tank was constructed in 1943 for the storage of LLLW.

\subsection{Characterization Summary}

The results for this tank are given in Tables 2.5.1 and 2.5.2.

\subsubsection{Aqueous liquid}

The results for this tank were similar to those for W-8. The VOA and SVOA did not reveal any significant levels of TCL constituents, except for the low levels of PAHs and phthalates in the sludge. The SVOA TIC, TBP, was estimated at $10 \mathrm{mg} / \mathrm{L}$ in L90. The recoveries of the surrogate standard compounds in $\mathrm{L} 90$ were all zero, suggesting either that an error had been made in surrogate standard spiking or that the matrix seriously interfered with the extraction. together.

Except for the sludges, the samples for tanks W-1, W-2, and W-4 were prepared 
Table 25.1. Analytical data for tank W-9

\begin{tabular}{|c|c|c|c|c|c|}
\hline \multirow{2}{*}{ Parameter } & \multirow{2}{*}{$\begin{array}{c}D L \\
(m g / L)\end{array}$} & \multirow{2}{*}{ L90 } & \multirow{2}{*}{ L92 } & \multicolumn{2}{|c|}{ RCRA limits } \\
\hline & & & & $(\mathrm{mg} / \mathrm{L})$ & $(\mathrm{mg} / \mathrm{kg})$ \\
\hline \multicolumn{6}{|c|}{ Physical properties and miscellaneous datus } \\
\hline Density $(g / m L)$ & & 1.017 & NA & & \\
\hline Depth (inches) & & 5 & NA & & \\
\hline Suspended solids $(\mathrm{mg} / \mathrm{mL})$ & 0.1 & $<0.1$ & NA & & \\
\hline TDS (mg/mL) & 0.1 & 21.2 & NA & & \\
\hline TOC $(L=m g / L, S=m g / k g)$ & 1.0 & 290 & 13,900 & & \\
\hline TS $(\mathrm{L}=\mathrm{mg} / \mathrm{mL}, \mathrm{S}=\mathrm{mg} / \mathrm{g})$ & 0.1 & 21.2 & 944 & & \\
\hline \multicolumn{6}{|c|}{$R C R A$ metals $(L=m g / L, S=m g / k g)$} \\
\hline Ag & 0.002 & $<0.002$ & 0.77 & 5.0 & 100 \\
\hline As & 0.40 & $<4$ & $<5$ & 5.0 & 100 \\
\hline $\mathbf{B a}$ & 0.02 & $<0.2$ & $(200)$ & 100 & 2,000 \\
\hline $\mathrm{Cd}$ & 0.01 & $<0.2$ & 4.9 & 1.0 & 200 \\
\hline $\mathrm{Cr}$ & 0.10 & 7.3 & 160 & 5.0 & 100 \\
\hline $\mathrm{Hg}$ & 0.005 & 0.06 & 40 & 0.2 & 4.0 \\
\hline $\mathrm{Ni}$ & 0.10 & $<1$ & 110 & NA & NA \\
\hline $\mathrm{Pb}$ & 0.50 & $<2$ & 620 & 5.0 & 100 \\
\hline $\mathrm{Se}$ & 0.05 & $<0.09$ & $<0.7$ & 1.0 & 20.0 \\
\hline $\mathrm{T} 1$ & 0.05 & $<0.09$ & $<0.7$ & NA & NA \\
\hline \multicolumn{6}{|c|}{ Process mesals ( $L=m g / L, S=m g / k g)$} \\
\hline $\mathrm{Si}$ & 1.0 & 2.53 & NA & & \\
\hline $\mathrm{U}$ & 0.1 & 2,390 & 25,800 & & \\
\hline \multicolumn{6}{|c|}{ Arions $(L=m g / L, s=m g / k g)$} \\
\hline $\mathrm{Cl}^{-}$ & 1.0 & 340 & $<100$ & & \\
\hline $\mathrm{Cn}$ & 0.01 & 0.14 & $\bullet$ & & \\
\hline $\mathrm{F}$ & 1.0 & $<50$ & $<100$ & & \\
\hline $\mathrm{NO}_{3} \cdot$ & 5.0 & 1,650 & * & & \\
\hline $\mathrm{PO}_{4}^{-2}$ & 5.0 & 2,600 & $<500$ & & \\
\hline $\mathrm{SO}_{4}^{-2}$ & 5.0 & 900 & $<500$ & & \\
\hline \multicolumn{6}{|c|}{ Alkalinity } \\
\hline $\mathrm{CO}_{3}^{-2}(\mathrm{~mol})$ & 0.01 & 0.03 & - & & \\
\hline $\mathrm{HCO}_{3}^{\circ}(\mathrm{mol})$ & 0.01 & 0.09 & - & & \\
\hline $\mathrm{OH}^{\circ}(\mathrm{mol})$ & 0.01 & $\cdot$ & * & & \\
\hline $\mathrm{pH}$ (units) & 0.1 & 10.2 & NA & & \\
\hline \multicolumn{6}{|c|}{ Radiochemical screaning ( $L=B q / \ln L, S=B q / 8)$} \\
\hline Gross alpha & & $6.0 \mathrm{E}+01$ & $1.1 \mathrm{E}+05$ & & \\
\hline Gross beta & & $7.1 E+04$ & $5.0 \mathrm{E}+06$ & & \\
\hline \multicolumn{6}{|c|}{ Besalgamma enitters $(L=B q / m L, S=B q / 8)$} \\
\hline${ }^{134} \mathrm{Cs}$ & & 8.5E+01 & $2.0 \mathrm{E}+01$ & & \\
\hline${ }^{137} \mathrm{Cs}$ & & $6.3 \mathrm{E}+04$ & $2.2 E+04$ & & \\
\hline${ }^{152} \mathrm{Eu}$ & & $\cdot$ & $2.3 \mathrm{E}+02$ & & \\
\hline${ }^{154} \mathrm{Eu}$ & & - & $4.4 \mathrm{E}+02$ & & \\
\hline${ }^{155} \mathrm{Eu}$ & & • & $5.2 \mathrm{E}+01$ & & \\
\hline${ }^{3} \mathrm{H}$ & & $1.0 \mathrm{E}+01$ & $0.0 \mathrm{E}+00$ & & \\
\hline${ }^{60} \mathrm{Co}$ & & $1.1 \mathrm{E}+02$ & $7.8 E+02$ & & \\
\hline${ }^{90} \mathrm{Sr}$ & & $3.7 \mathrm{E}+02$ & $2.2 E+06$ & & \\
\hline
\end{tabular}


Waste Characterization Data for ORNL Inactive LLLW Tanks Rev. 0 / June 1992

Table 25.1 (onntinued)

\begin{tabular}{|c|c|c|c|c|c|}
\hline \multirow{2}{*}{ Parameter } & \multirow{2}{*}{$\begin{array}{c}D L \\
(m g / L)\end{array}$} & \multirow{2}{*}{ L90 } & \multirow{2}{*}{ L92 } & \multicolumn{2}{|c|}{ RCRA limits } \\
\hline & & & & $(\mathrm{mg} / \mathrm{L})$ & $(\mathrm{mg} / \mathrm{kg})$ \\
\hline \multicolumn{6}{|c|}{ Alpha emituess $(L=B$ q $/ \mathrm{mL}, S=B q(g)$} \\
\hline${ }^{233} \mathrm{U}$ & & $<3$ & $3.1 E+03$ & & \\
\hline${ }^{238} \mathrm{Pu}$ & & $<3$ & $2.2 E+04$ & & \\
\hline${ }^{239} \mathrm{Pu}$ & & $<3$ & $9.2 E+03$ & & \\
\hline${ }^{241} \mathrm{Am}$ & & & $7.2 \mathrm{E}+03$ & & \\
\hline${ }^{244} \mathrm{Cm}$ & & $<3$ & $5.4 \mathrm{E}+04$ & & \\
\hline${ }^{252} \mathrm{Cf}$ & & $<3$ & - & & \\
\hline
\end{tabular}

$\mathrm{DL}=$ Detection limit

Table 25.2 Organic anatytical data hits for tank W-9

\begin{tabular}{|c|c|c|c|c|c|}
\hline Parameter & $\begin{array}{l}\text { CRQL } \\
(\mu \mathrm{g} / \mathrm{L})\end{array}$ & L90 & L92 & $\begin{array}{c}\text { RCRA limit } \\
(\mu \mathrm{g} / L)\end{array}$ & $\begin{array}{l}\text { CRQL } \\
(\mu \mathrm{g} / \mathrm{kg})\end{array}$ \\
\hline \multicolumn{6}{|c|}{ Volatile onganic compound anatyois $(L=\mu g / L, S=n g / k g)$} \\
\hline 4-Methyl-2-pentanone & 10 & 11 & & & \\
\hline Acetone & 10 & $70 \mathrm{~B}$ & & & \\
\hline Benzene & 5 & $2 \mathrm{~J}$ & & 500 & \\
\hline Chlorobenzene & 5 & 5 & & 100,000 & \\
\hline Chloroform & 5 & 44 & & 6,000 & \\
\hline Methylene chloride & 5 & $13 \mathrm{~B}$ & & & \\
\hline Tetrachloroethene & 5 & $4 \mathrm{~J}$ & & 700 & \\
\hline Toluene & 5 & $9 \mathrm{~B}$ & & & \\
\hline Trichloroethene & 5 & 5 & & 500 & \\
\hline \multicolumn{6}{|c|}{ Semivolatile compound anabuir ( $L=m g / L, S=\mu g / k g)$} \\
\hline Bis(2-ethylhexyl)phthalate & 10 & & 60,000 & & 330 \\
\hline Di-n-butylphthalate & 10 & $20 \mathrm{~J}$ & $760 \mathrm{~J}$ & & 330 \\
\hline Diethylphthalate & 10 & & $450 \mathrm{~J}$ & & 330 \\
\hline Fluoranthene & 10 & & $1,100 \mathrm{~J}$ & & 330 \\
\hline Naphthalene & 10 & & $370 \mathrm{~J}$ & & 330 \\
\hline Phenanthrene & 10 & & $1,300 \mathrm{~J}$ & & 330 \\
\hline Pyrene & 10 & & $850 \mathrm{~J}$ & & 330 \\
\hline
\end{tabular}

$\mathrm{CRQL}=$ contract required quantitation limit 


\section{TANK W-10}

\subsection{Tank Description}

Tank W-10 is a 170,000 -gal Gunite tank located in the Bethel Valley South Tank Farm. The underground tank was constructed in 1943 for the storage in LLLW.

\subsubsection{Characterization Summary}

The results for this tank are given in Tables 2.6.1 and 2.6.2.

\subsubsection{Aqueous liquid}

All the ar ueous samples for tank W-10 were processed as a group. DAI-GC indicated the presence of 30 to $40 \mathrm{mg} / \mathrm{L}$ of methanol. The acetone determined by DAI-GC was qualitatively confirmed by VOA, but the latter indicated much lower levels. The presence of 2-butanone was not verified by VOA. Except for the methanol, there were only very low levels of volatile organic compounds in the aqueous liquids, and in L93, none were detected at all. This is consistent with TOC in L93 $(8.7 \mathrm{mg} / \mathrm{L})$ being much lower than in L94 and L95 (86.4 and $54.6 \mathrm{mg} / \mathrm{L}$, respectively).

The only TCL semivolatile organic compound detected in the liquids was benzoic acid, in $\mathrm{L} 94$ and $\mathrm{L} 95$ ( 400 and $2900 \mu \mathrm{g} / \mathrm{L}$, respectively). The naphthalene detected in $\mathrm{L} 95$ was very low $(20 \mu \mathrm{g} / \mathrm{L})$. TBP was indicated as a TIC only in $\mathrm{L} 94$, at $2 \mathrm{mg} / \mathrm{L}$.

\subsubsection{Sludge}

Like the sludges from the other tanks, the sludges from tank W-10 contained PAHs and phthalates, with $\mathrm{H} 120$ having higher concentrations of PAHs than S96, which is consistent with their TOC levels $(14,600$ and $8180 \mathrm{mg} / \mathrm{kg}$, respectively). The SVOA TIC, ethylphenylethanone, was estimated at 200 and $2300 \mu \mathrm{g} / \mathrm{L}$ in L94 and L95, respectively, and 9 and 40 $\mathrm{mg} / \mathrm{kg}$ in S96 and H120, respectively. TBP was detected as a TIC in the sludges at 60 and 300 $\mathrm{mg} / \mathrm{kg}$ in $\mathrm{S} 96$ and $\mathrm{H} 120$, respectively. The hard sludges appear to contain generally higher concentrations of organic compounds than do the soft sludges.

The acidic matrix spike recoveries for L93 were very poor; however, the acidic surrogate standard recoveries were good. The reason for this discrepancy is not clear. Two of the acidic compound and two of the neutral compound surrogate standard recoveries were low for S96 but were better for the hard sludge, H120. The hard sludges exhibited better surrogate standard and matrix spike recoveries than did the soft sludges. The reasons for these findings are not clear but may be related to the more aqueous character of the soft sludges. 
Waste Characterization Data for ORNL Inactive LLLW Tanks

Rev. 0/June 1992

Section $2.6 /$ page 2 of 3

Table 26.1. Analytical data for tank W-10

\begin{tabular}{ccccc}
\hline Parameter & $\mathrm{D} \mathrm{L}$ \\
$(\mathrm{mg} / \mathrm{L})$ & $\mathrm{S} \%$ & RCRA limits \\
\hline
\end{tabular}

Density $(\mathrm{g} / \mathrm{mL})$

Depth (inches)

Suspended solids (mg/mL)

TDS $(\mathrm{mg} / \mathrm{mL})$

TOC $(\mathrm{L}=\mathrm{mg} / \mathrm{L}, \mathrm{S}=\mathrm{mg} / \mathrm{kg})$

$T S(\mathrm{~L}=\mathrm{mg} / \mathrm{mL}, \mathrm{S}=\mathrm{mg} / \mathrm{g})$

$\mathrm{Ag}$

As

$\mathrm{Ba}$

$\mathrm{Cd}$

$\mathrm{Cr}$

$\mathrm{Hg}$

$\mathrm{Ni}$

$\mathrm{Pb}$

$\mathrm{Se}$

TI

Physical properties and miscellaneons data

$\begin{array}{rr} & \text { NA } \\ & \text { NA } \\ 0.1 & \text { NA } \\ 0.1 & \text { NA } \\ 1.0 & 8180 \\ 0.1 & 449\end{array}$

RCRA metals $(L=m g / L, S=m g / k g)$

$\begin{array}{lllll}0.002 & 0.79 & 5.0 & \ldots & 100\end{array}$

$\begin{array}{rrrr}0.40 & 5.7 & 5.0 & 100\end{array}$

$\begin{array}{llll}0.02 & (94) & 100.0 & 2000\end{array}$

$\begin{array}{rrrr}0.01 & 4.7 & 1.0 & 20.0\end{array}$

$\begin{array}{llll}0.10 & (140) & 5.0 & 100\end{array}$

$\begin{array}{llll}0.005 & 48 & 0.2 & 4.0\end{array}$

$\begin{array}{llll}0.10 & 83 & \text { NA } & \text { NA }\end{array}$

$\begin{array}{llll}0.50 & 480 & 5.0 & 100\end{array}$

$\begin{array}{llll}0.05 & (<0.9) & 1.0 & 20.0\end{array}$

$\begin{array}{llll}0.05 & (<0.9) & \text { NA }\end{array}$

Process metals ( $L=m g / L, S=m g / k g)$

$\mathrm{Si}$

1.0

NA

0.1

10,800

$\mathrm{Cl}^{\cdot}$

$\mathrm{Cn}$

$\mathrm{F}$

$\mathrm{NO}_{3}{ }^{-}$

$\mathrm{PO}_{4}^{-2}$

$\mathrm{SO}_{4}^{-2}$

$\mathrm{CO}_{3}^{-2}(\mathrm{~mol})$

$\mathrm{HCO}_{3}^{\circ}$ (mol)

$\mathrm{pH}$ (units)

Gross alpha

Gross beta

$\begin{array}{lr} & \text { Anions }(L=m g / L, S \\ 1.0 & <120 \\ 0.01 & * \\ 1.0 & <120 \\ 5.0 & < \\ 5.0 & <600 \\ 5.0 & <600 \\ & \text { Alkalinity } \\ 0.01 & \text {. } \\ 0.01 & \text { NA } \\ 0.1 & \end{array}$

Radiochemical screening $(L=B q / m L, S=B q / g)$

$$
8.4 \mathrm{E}+04
$$

$5.3 \mathrm{E}+06$

${ }^{134} \mathrm{Cs}$

${ }^{137} \mathrm{Cs}$

Bealgannma amitress $(L=B q / m I, S=B q / g)$

${ }^{152} \mathrm{Eu}$

$8.6 \mathrm{E}+05$

$4.6 \mathrm{E}+03$

$8.0 \mathrm{E}+03$

$1.4 \mathrm{E}+02$

$1.3 \mathrm{E}+04$

${ }^{60} \mathrm{Co}$

${ }^{90} \mathrm{Sr}$

$8.6 \mathrm{E}+06$ 
Waste Characterization Data for ORNL Inactive LLLW Tanks Rev. () / June 1992

Table 2.6.1 (continued)

\begin{tabular}{|c|c|c|c|c|}
\hline \multirow{2}{*}{ Parameter } & \multirow{2}{*}{$\begin{array}{c}D L \\
(\mathrm{mg} / \mathrm{L})\end{array}$} & \multirow{2}{*}{ S96 } & \multicolumn{2}{|c|}{ RCRA limits } \\
\hline & & & $(\mathrm{mg} / \mathrm{L})$ & $(\mathrm{mg} / \mathrm{kg})$ \\
\hline \multicolumn{5}{|c|}{ Alpha emitters $(L=B q$ tri $L, S=B q$ tg) } \\
\hline${ }^{233} \mathrm{U}$ & & $<50$ & & \\
\hline${ }^{238} \mathrm{Pu}$ & & $2.9 \mathrm{E}+04$ & & \\
\hline${ }^{238} \mathrm{U}$ & & $\cdot$ & & \\
\hline${ }^{239} \mathrm{Pu}$ & & $8.2 \mathrm{E}+03$ & & \\
\hline${ }^{241} \mathrm{Am}$ & & $4.5 \mathrm{E}+03$ & & \\
\hline${ }^{244} \mathrm{Cm}$ & & $4.7 \mathrm{E}+04$ & & \\
\hline${ }^{252} \mathrm{Cf}$ & & $<50$ & & \\
\hline
\end{tabular}

Table 26.2. Organic analytical data hits for tank W-10

\begin{tabular}{lccccccccccc}
\hline Parameter & $\begin{array}{c}\mathrm{CRQL} \\
(\mu \mathrm{g} / \mathrm{L})\end{array}$ & $\mathrm{H} 120$ & $\mathrm{~L} 93$ & $\mathrm{L94}$ & L94B & L95 & S96 & $\begin{array}{c}\text { RCRA } \\
\text { limit } \\
(\mu \mathrm{g} / \mathrm{L})\end{array}$ & $\begin{array}{c}\text { CRQL } \\
(\mu \mathrm{g} / \mathrm{kg})\end{array}$ \\
\hline
\end{tabular}

Acetone

Ethyl alcohol

Methyl alcohol

Methyl ethyl ketone

N.Butyl alcohol

1,2-Dichloroethene (total)

4-Methyl-2-pentanone

Acetone

Benzene

Chlorobenzene

Chloroform

Methylene chloride

Tetrachloroethene

Toluene

Trichloroethene

Benzo(a)anthracene

Benzo(a)pyrene

Benzo(b) lluoranthene

Benzo(g,h,i)perylene

Benzoic acid

Bis(2-ethylhexyl)phthalate

Chrysene

Di-n-butylphthalate

Fluoranthene

Naphthalene

Phenanthrene

Pyrene
$D A I-G C$ andalyois $(L=\mu g / L, S=\mu g / \mathrm{kg})$

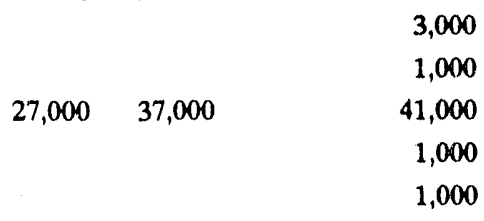

Volatile onganic compound anabusis ( $L=\mu g / L, S=\mu g / k g)$
5

10

10

5

5

5

5

5

5

5

Samivolatile compound anahusis $(L=\mu g / L, S=\mu g / \mathrm{kg})$

10

$1,900 \mathrm{~J}$

$2,200 \mathrm{~J}$

$870 \mathrm{~J}$

$400 \mathrm{~J}$

$770 \mathrm{~J}$

$8,000 \mathrm{~J}$

$270 \mathrm{~J}$

$5,100 \mathrm{~J}$

$8,100 \mathrm{~J}$
11

31

$23 \mathrm{~B}$

$97 \mathrm{~B}$

$6 \mathrm{~B} \quad 38 \mathrm{BM} \quad 22 \mathrm{~B}$

$5 \mathrm{~B}$

16

$13 \mathrm{~B} \quad 19 \mathrm{~B} \quad 12 \mathrm{~B}$

$21 \quad 48$

$12 \quad 26 \mathrm{~B}$

$27 \quad 81$

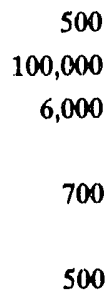

$660 \mathrm{~J}$

330

330

330

330

1600

330

330

330

330

$20 \mathrm{~J}$

330

330

$1,700 \mathrm{~J}$

$1,400 \mathrm{~J}$ 


\section{TANK W-11}

\subsubsection{Tank Description}

Tank W-11 is a 1500 -gal Gunite tank located in the Bethel Valley South Tank Farm. The underground tank was constructed in 1943 to serve as a waste collection and monitoring tank for research laboratories in Building 3550.

\subsection{Characterization Summary}

The results for this tank are shown in Tables 2.7.1 and 2.7.2.

\subsubsection{Aqueous liquid}

The volatile organic compounds in tank W-11 samples were very low. Except for sludge samples, no SVOA extracts were submitted for GC-MS analysis because the GC screening did not detect sufficient concentrations of organic compounds for GC. The screening data are consistent with the TOC levels for these samples, which are the lowest TOC levels taken from any of the tanks in this study $(2.0,<0.1$, and $<0.1 \mathrm{mg} / \mathrm{L}$ for $\mathrm{L3}, \mathrm{L} 114$, and $\mathrm{L} 115$, respectively). Samples L3 and L115 were sequentially collected and are useful as duplicates, within the usual errors of sampling. The VOA results are in reasonable agreement, considering the very low concentrations $(2-30 \mu \mathrm{g} / \mathrm{L})$.

\subsubsection{Sludge}

The sludge contained several PAHs at concentrations as high as hundreds of $\mathrm{mg} / \mathrm{kg}$, as shown in Table 2.7.2. The presence of these species was quite unexpected, since PAH-bearing wastes, such as tars, were not known to be disposed of in the tank. The PAHs were not detected in the liquid samples, as expected from their low aqueous solubilities (low $\mu \mathrm{g} / \mathrm{L}$ for the five-ring $\mathrm{PAH}$ ), and they selectively partitioned into the sludge. This trend was observed for other sludge samples. Phthalates were also present. 
Waste Characterization Data for ORNL Inactive LLLW Tanks

Talte 27.1. Analynical data for taak W-11

\begin{tabular}{|c|c|c|c|c|c|c|c|}
\hline \multirow{2}{*}{ Parameter } & \multirow{2}{*}{$\begin{array}{c}D L \\
(m g / L)\end{array}$} & \multirow{2}{*}{$\mathrm{HS}$} & \multirow{2}{*}{ L114 } & \multirow{2}{*}{ L115 } & \multirow{2}{*}{ L3 } & \multicolumn{2}{|c|}{ RCRA limits } \\
\hline & & & & & & $(m g l)$ & $(\mathrm{Hg} / \mathrm{kg})$ \\
\hline \multicolumn{8}{|c|}{ Physical propurtias and miscellaneous dara } \\
\hline Density $(g / m L)$ & & 2,080 & 1.000 & 0.998 & 0.999 & & \\
\hline Depth (inches) & & NA & 24 & 4 & 4 & & \\
\hline Suspended solids (ng/mL) & 0.1 & NA & 0.2 & 0.2 & 0.2 & & \\
\hline $\operatorname{TDS}(\mathrm{mg} / \mathrm{mL})$ & 0.1 & NA & 0.1 & 0.1 & 0.1 & & \\
\hline $\operatorname{TOC}(\mathrm{L}=\mathrm{mg} / \mathrm{L}, \mathrm{S}=\mathrm{mg} / \mathrm{gg})$ & 1.0 & $\cdot$ & $<0.1$ & $<0.1$ & $<0.1$ & & \\
\hline TS $(\mathrm{L}=m \mathrm{~g} / \mathrm{mL}, \mathrm{S}=\mathrm{mg} / \mathrm{g})$ & 0.1 & 1,200 & 0.3 & 0.3 & 0.3 & & \\
\hline \multicolumn{8}{|c|}{ RCRA mesals ( $L=m g / L, S=m g / k)$} \\
\hline Af & 0.002 & 2.4 & $<0,102$ & $(<0.002)$ & $(<0.002)$ & 5.0 & 100 \\
\hline As & 0.40 & $(1.8)$ & $<0.8$ & $<0.8$ & $<0.8$ & 5.0 & 100 \\
\hline $\mathrm{Ba}$ & 0.02 & 64 & 0.05 & $<0.04$ & $<0.04$ & 100 & 2,000 \\
\hline $\mathrm{Cd}$ & 0.01 & $(1.2)$ & $<0.02$ & $<0.02$ & $<0.02$ & $i .0$ & 20.0 \\
\hline $\mathrm{Cr}$ & 0.10 & 670 & $<0.1$ & $<0.1$ & $<0.1$ & 5.0 & 100 \\
\hline $\mathrm{Hg}_{\mathrm{g}}$ & 0.005 & 17 & $<0.09$ & $<0.01$ & $<0.04$ & 0.2 & 4.0 \\
\hline $\mathrm{Nj}$ & 0.10 & 10 & $<0.2$ & $<0.2$ & $<0.2$ & NA & NA \\
\hline $\mathrm{Pb}$ & 0.50 & $(14.004))$ & $<1$ & $<1$ & $<1$ & 5.0 & 100 \\
\hline Se & 0.05 & $<0.3$ & $<0.09$ & $<0.09$ & $<0.09$ & 1.0 & 20.0 \\
\hline $\mathrm{T}$ & 0.05 & $<0.3$ & $(<0.09)$ & $(<0,09)$ & $(<0.09)$ & NA & NA \\
\hline \multicolumn{8}{|c|}{ Proxess mexals ( $L=m g / h, S=m g / k g)$} \\
\hline Si & 1.0 & NA & 7.29 & 7.03 & 6.91 & & \\
\hline $\mathrm{U}$ & 0.1 & $\cdot$ & 8.82 & 8.88 & 12.1 & & \\
\hline \multicolumn{8}{|c|}{ Anions $(L=m g / L, S=m g / k g)$} \\
\hline $\mathrm{Cl}$ & 1.0 & - & $<1$ & $<1$ & $<1$ & & \\
\hline $\mathrm{Cn}$ & 0.01 & • & $<0.04$ & $<0.04$ & $<0.04$ & & \\
\hline$F^{\circ}$ & 1.0 & • & $<1$ & $<1$ & $<1$ & & \\
\hline $\mathrm{NO}_{3}$ & 5.0 & • & $<5$ & $<5$ & $<5$ & & \\
\hline $\mathrm{PO}_{4}^{-2}$ & 5.0 & * & $<5$ & $<5$ & $<5$ & & \\
\hline $\mathrm{SO}_{4}^{-2}$ & 50 & • & $<5$ & $<5$ & $<5$ & & \\
\hline \multicolumn{8}{|c|}{ Ablatining } \\
\hline $\mathrm{pH}$ (units) & 0.01 & $\mathrm{NA}$ & 7.7 & 7.8 & 8.0 & & \\
\hline \multicolumn{8}{|c|}{ Radiochumical xcreening $(L=B q \operatorname{mon} L, S=B q / g)$} \\
\hline Gross aipha & & $\bullet$ & $<1$ & $<1$ & $<1$ & & \\
\hline Gross beta & & - & $<20$ & $<20$ & $<20$ & & \\
\hline \multicolumn{8}{|c|}{ Bectafgariane onvituors $\left(L=B q / m L_{4} S=B q / q\right)$} \\
\hline${ }^{137} \mathrm{Cs}_{\mathrm{s}}$ & & $\cdot$ & $<20$ & $<20$ & $<20$ & & \\
\hline${ }^{14} \mathrm{C}$ & & - & 1.0E:-01 & $1.0 \mathrm{E}-02$ & $2.0 \mathrm{E}-101$ & & \\
\hline${ }^{3} \mathrm{H}$ & & • & S.OE - 01 & S.OE.01 & $6.0 \mathrm{E}-01$ & & \\
\hline${ }^{\infty} \mathrm{Co}$ & & * & $<20$ & 4.20 & $<20$ & & \\
\hline $\mathrm{Sr}_{r}$ & & $\cdot$ & $21 \mathrm{E}+01$ & $4.2 E+01$ & $2.0 \mathrm{E}+01$ & & \\
\hline
\end{tabular}


Waste Characterization Data for ORNL Inactive LLLW Tanks Rev. 0 / June 1992

Table 27.2. Organic analytical data hits for tunk W-11

\begin{tabular}{|c|c|c|c|c|c|c|}
\hline Parameter & $\begin{array}{l}\text { CRQL } \\
(\mu \mathcal{L} L)\end{array}$ & $\mathrm{H5}$ & 13 & L114 & L115 & $\begin{array}{c}\text { RCRA } \\
\text { limit } \\
(\mu g / L)\end{array}$ \\
\hline \multicolumn{7}{|c|}{ Volatile orgunic compound anabois ( $L=n g / L, S=n g / k g)$} \\
\hline Acetone & 10 & & $27 \mathrm{~B}$ & $21 \mathrm{~B}$ & $15 \mathrm{~B}$ & \\
\hline Chlorobenzene & 5 & & $5 \mathrm{~J}$ & $3 \mathrm{~J}$ & $2 \mathrm{~J}$ & 100,000 \\
\hline Methylene chloride & 5 & & $7 \mathrm{~B}$ & 8 B & $13 \mathrm{~B}$ & \\
\hline Toluene & 5 & & $23 \mathrm{~B}$ & $24 B$ & $13 \mathrm{~B}$ & \\
\hline Trichloroethene & 5 & & $4 \mathrm{~J}$ & & & 500 \\
\hline \multicolumn{7}{|c|}{ Senivolatile compound mabuis ( $L=\mu g / L, S=$ Re/hg) } \\
\hline Benzo(a)anthracene & 10 & 44,000 & & & & \\
\hline Benzo(a)pyrene & 10 & 64,000 & & & & \\
\hline Benzo(b)fluoranthene & 10 & $160,000 \mathrm{E}$ & & & & \\
\hline Benzo(g,h,i)perylene & 10 & 22,000 & & & & \\
\hline Bis(2-ethylhexyl)phtha & 10 & 38,000 & & & & \\
\hline Chrysene & 10 & 130,000 & & & & \\
\hline Di-n-butylphthalate & 10 & 17,400 & & & & \\
\hline Fluoranthene & 10 & $240,000 \mathrm{E}$ & & & & \\
\hline Indeno(1,2,3-cd)pyrene & 10 & 42,000 & & & & \\
\hline Phenanthrene & 10 & 24,000 & & & & \\
\hline Pyrene & 10 & 90,000 & & & & \\
\hline
\end{tabular}

$\mathrm{CRQL}=$ contract required quantitation limit 


\section{TANK T-1}

\subsection{Tank Description}

Tank T-1 is a 15,000-gal carbon steel tank located in Melton Valley at the Old Hydrofracture Facility (OHF). The underground tank was installed at the site in 1963 to store LLLW for mixing into grout for waste injection by hydrofracture.

\subsection{Characterization Summary}

The results for this tank are shown in Tables 2.8.1 and 2.8.2.

\subsubsection{Aqueous liquid}

Very little organic matter was detected in the samples from this tank. VOA apparently revealed high levels of methylene chloride in both the sample and the blank. These high levels can be attributed to contamination of the solid sorbent traps during storage. When the quantitation procedure was modified to permit analysis of traps within $48 \mathrm{~h}$ of receipt, the level of methylene chloride in the blank was reduced to acceptable levels. In the SVOA extraction, the methylene chloride and aqueous layers were difficult to separate for samples L35 and L36. Sample L36 required centrifugation to achieve separation. However, surrogate standard and matrix spike recoveries were good. There were no SVOA TCL hits in the aqueous liquids.

SVOA TICs included diphenyloxazole $(300-400 \mu \mathrm{g} / \mathrm{L})$, hexadecanoic acid $(300 \mu \mathrm{g} / \mathrm{L}$ in one sample), and TBP (30-40 mg/L). TBP was often the major organic compound detected.

\subsubsection{Sludge}

Of the SVOA TCL compounds in the sludge, only phthalates were detected. SVOA TICs in the sludge included TBP at $2 \mathrm{mg} / \mathrm{kg}$. 
Waste Characterization Data for ORNL Inactive LLLW Tanks

Table 28.1. Analytical data for cank T-1

\begin{tabular}{|c|c|c|c|c|c|c|}
\hline \multirow{2}{*}{ Parameter } & \multirow{2}{*}{$\begin{array}{c}D L \\
(\mathrm{mg} / \mathrm{L})\end{array}$} & \multirow{2}{*}{ L35 } & \multirow{2}{*}{ L.36 } & \multirow{2}{*}{ \$37 } & \multicolumn{2}{|c|}{ RCRA limits } \\
\hline & & & & & $(\mathrm{mg} / \mathrm{L})$ & $(\mathrm{mg} / \mathrm{kg})$ \\
\hline \multicolumn{7}{|c|}{ Physical properties and miscellaneous data } \\
\hline Density $(g / m L)$ & & 1.005 & 1.007 & NA & & \\
\hline Depth (inches) & & 4 & 48 & NA & & \\
\hline Suspended solids (mg/mL) & 0.1 & 0.8 & $<0.1$ & NA & & \\
\hline $\mathrm{TDS}(\mathrm{mg} / \mathrm{mL})$ & 0.1 & 6.4 & 6.9 & NA & & \\
\hline $\operatorname{TOC}(\mathrm{L}=\mathrm{mg} / \mathrm{L}, \mathrm{S}=\mathrm{mg} / \mathrm{kg})$ & 1.0 & 836.0 & 790.0 & 18,600 & & \\
\hline $\mathrm{TS}(\mathrm{L}=\mathrm{mg} / \mathrm{mL}, \mathrm{S}=\mathrm{mg} / \mathrm{g})$ & 0.1 & 7.2 & 6.9 & 921 & & \\
\hline \multicolumn{7}{|c|}{$R C R A$ mesals $(L=m g / L, S=m g / k g)$} \\
\hline Ag & 0.002 & 0.005 & 0.005 & 2.1 & 5.0 & 100 \\
\hline As & 0.40 & $<0.8$ & $<0.8$ & $<2$ & 5.0 & 100 \\
\hline $\mathrm{Ba}$ & 0.02 & $<0.04$ & 0.05 & 88 & 100 & 2,000 \\
\hline $\mathrm{Cd}$ & 0.01 & $<0.02$ & $<0.02$ & 12.9 & 1.0 & 20.0 \\
\hline $\mathrm{Cr}$ & 0.10 & 0.29 & 0.18 & $(130)$ & 5.0 & 100 \\
\hline $\mathrm{Hg}_{\mathrm{g}}$ & 0.005 & 0.06 & 0.07 & 74 & 0.2 & 4.0 \\
\hline $\mathrm{Ni}$ & 0.10 & $<0.2$ & $<0.2$ & 190 & NA & NA \\
\hline $\mathrm{Pb}$ & 0.50 & $<1$ & $<1$ & $(860)$ & 5.0 & 100 \\
\hline $\mathrm{Se}$ & 0.05 & $<0.2$ & $<0.2$ & $(<2)$ & 1.0 & 20.0 \\
\hline $\mathrm{T}$ & 0.05 & $<0.2$ & $<0.2$ & 1.7 & NA & NA \\
\hline \multicolumn{7}{|c|}{ Process metals $(L=m g / h, S=m g / t g)$} \\
\hline $\mathrm{Si}$ & 1.0 & 9.34 & 6.81 & NA & & \\
\hline $\mathrm{U}$ & 0.10 & 172 & 175 & 2800 & & \\
\hline \multicolumn{7}{|c|}{ Anions ( $L=m g / L, S=m g / k g)$} \\
\hline $\mathrm{Cl}^{\circ}$ & 1.0 & 7.8 & 8.0 & - & & \\
\hline $\mathrm{Cn}$ & 0.01 & $\cdot$ & 0.22 & - & & \\
\hline $\mathrm{F}$ & 1.0 & 1.0 & 1.0 & * & & \\
\hline $\mathrm{NO}_{3}$ & 5.0 & 16 & 17 & $\cdot$ & & \\
\hline $\mathrm{PO}_{4}^{-2}$ & 5.0 & $<5$ & $<5$ & - & & \\
\hline $\mathrm{SO}_{4}^{-2}$ & 5.0 & 7.5 & 8.1 & - & & \\
\hline \multicolumn{7}{|c|}{ Alkalinuiny } \\
\hline $\mathrm{CO}_{3}^{-2}(\mathrm{~mol})$ & 0.01 & 0.01 & 0.01 & - & & \\
\hline $\mathrm{HCO}_{3}(\mathrm{~mol})$ & 0.01 & 0.04 & 0.04 & * & & \\
\hline pH (units) & 0.1 & 9.7 & 9.7 & NA & & \\
\hline \multicolumn{7}{|c|}{ Radiochemical screaning ( $L=B$ giml, $S=B q$ /g) } \\
\hline Gross alpha & & $1.9 \mathrm{E}+02$ & $2.1 \mathrm{E}+02$ & $6.5 E+05$ & & \\
\hline Gross beta & & $8.1 E+04$ & $7.8 \mathrm{E}+04$ & $5.9 \mathrm{E}+07$ & & \\
\hline \multicolumn{7}{|c|}{ 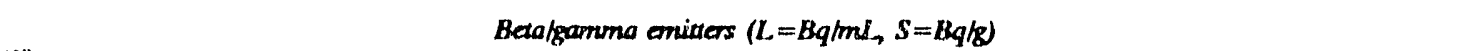 } \\
\hline${ }^{13 \prime} \mathrm{Cs}$ & & $7.4 \mathrm{E}+04$ & $7.5 \mathrm{E}+0$ & $3.9 \mathrm{E}+05$ & & \\
\hline${ }^{110} \mathrm{C}$ & & $\cdot$ & $\cdot$ & $4.8 \mathrm{E}+01$ & & \\
\hline${ }^{152} \mathrm{Eu}$ & & - & - & $1.4 E+05$ & & \\
\hline${ }^{154} \mathrm{Eu}$ & & - & - & $1.2 \mathrm{E}+05$ & & \\
\hline${ }^{15 s} \mathrm{Eu}$ & & - & - & $2.3 \mathrm{E}+04$ & & \\
\hline${ }^{3} \mathrm{H}$ & & $7.1 \mathrm{E}+01$ & $7.1 \mathrm{E}+01$ & $2.6 \mathrm{E}+01$ & & \\
\hline${ }^{60} \mathrm{Co}$ & & $<50$ & $<50$ & $2.6 \mathrm{E}+05$ & & \\
\hline${ }^{90} \mathrm{Sr}$ & & $3.3 \mathrm{E}+03$ & $3.4 \mathrm{E}+03$ & $3.2 \mathrm{E}+07$ & & \\
\hline
\end{tabular}


Waste Characterization Data for ORNL Inactive LLLW Tanks Rev. 0 / June 1992

Table 2.8.1 (continued)

\begin{tabular}{|c|c|c|c|c|c|c|}
\hline \multirow{2}{*}{ Parameter } & \multirow{2}{*}{$\begin{array}{c}\mathrm{DL} \\
(\mathrm{mg} / \mathrm{L})\end{array}$} & \multirow{2}{*}{$\mathbf{L 3 5}$} & \multirow{2}{*}{1.36} & \multirow{2}{*}{$\$ 37$} & \multicolumn{2}{|c|}{ RCRA limits } \\
\hline & & & & & $(\mathrm{mg} / \mathrm{L})$ & $(\mathbf{m g} / \mathbf{k g})$ \\
\hline \multicolumn{7}{|c|}{ Alpha emitters $(L=B q$ im $L, S=B q / g)$} \\
\hline${ }^{233} \mathrm{U}$ & & $1.8 \mathrm{E}+02$ & $2.0 \mathrm{E}+02$ & $\cdot$ & & \\
\hline${ }^{238} \mathrm{Pu}$ & & - & $\cdot$ & $3.4 \mathrm{E}+04$ & & \\
\hline
\end{tabular}

$\mathrm{DL}=$ Detection limit

Table 28.2 Onganic analytical data hits for tank $T-1$

\begin{tabular}{|c|c|c|c|c|c|c|}
\hline Parameter & $\begin{array}{l}\text { CRQL } \\
(\mu g / L)\end{array}$ & L.35 & L36 & S37 & $\begin{array}{c}\text { RCRA } \\
\text { limit } \\
(\mu g / L)\end{array}$ & $\begin{array}{c}\text { CRQL } \\
(\mu \mathrm{g} / \mathrm{kg})\end{array}$ \\
\hline \multicolumn{7}{|c|}{ Volatile orgmic compound anabswis ( $L=\mu g / L, S=\mu g / \mu q)$} \\
\hline Acetone & 10 & $51 \mathrm{~B}$ & $27 \mathrm{~B}$ & & & \\
\hline Benzene & 5 & 130 & $5 \mathrm{~J}$ & & 500 & \\
\hline Carbon disulfide & 5 & 91 & & & & \\
\hline Carbon tetrachloride & 5 & $2 \mathrm{~J}$ & & & 500 & \\
\hline Chloroform & 5 & $12 \mathrm{~B}$ & $6 \mathrm{~B}$ & & 6000 & \\
\hline Methylene chloride & 5 & $1300 \mathrm{BE}$ & $970 \mathrm{BE}$ & & & \\
\hline Toluene & 5 & $71 \mathrm{~B}$ & $12 \mathrm{~B}$ & & & \\
\hline Trichlorocthene & 5 & $4 \mathrm{~J}$ & & & 500 & \\
\hline \multicolumn{7}{|c|}{ Seminolatile compound anabsir $(L=m g / L, S=\mu g / \mathrm{kg})$} \\
\hline Bis(2-ethylhexyl)phthalate & 10 & $400 \mathrm{~J}$ & $190 \mathrm{~J}$ & & & \\
\hline Di-n-butylphthalate & 10 & $75 \mathrm{~J}$ & $76 \mathrm{~J}$ & $3500 \mathrm{~J}$ & & 330 \\
\hline
\end{tabular}




\section{TANK T-2}

\subsubsection{Tank Description}

Tank T-2 is a 15,000-gal carbon steel tank located in Melton Valley at the OHF. The underground tank was installed at the site in 1963 to store LLLW for mixing into grout for waste injection by hydrofracture.

\subsubsection{Characterization Summary}

The results for this tank are shown in Tables 2.9.1 and 2.9.2.

\subsubsection{Aqueous liquid}

Very little volatile organic matter was detected in the aqueous liquids. Methylene chloride was a probable contaminant, as it was for tank T-1. However, SVOA showed the presence of 2-nitrophenol, 2,4-dichlorophenol, and 2,4,5-trichlorophenol in the liquids. These compounds could represent chemical reactions occurring in the tanks.

SVOA TICs in the three liquids included TBP at $20 \mathrm{mg} / \mathrm{L}$ and tetramethylbutylphenoxyethoxyethanol at $800-1000 \mu \mathrm{g} / \mathrm{L}$. Some foaming was observed in samples L.39 and L112 upon acidification.

Samples L.38 and L112, which were collected sequentially, are duplicates within the limits of field sampling. The agreement between the results for the two samples is quite good, especially considering the low levels determined.

\subsubsection{Sludge}

The sludge contained the usual phthalates and also naphthalene. The latter could be a component of liquid scintillator solutions. TBP was also detected in the sludge, but the amount could not be estimated because the mass spectrum of the chromatographic peak indicated coeluting interferences. 
Waste Characterization Data for ORNL Inactive LLLW Tanks

Table 29.1. Analytical data for tank $\mathrm{T}-2$

\begin{tabular}{|c|c|c|c|c|c|c|c|}
\hline \multirow{2}{*}{ Parameter } & \multirow{2}{*}{$\begin{array}{c}D L \\
(\mathrm{mg} / \mathrm{L})\end{array}$} & \multirow{2}{*}{ L112 } & \multirow{2}{*}{ L.38 } & \multirow{2}{*}{ L39 } & \multirow{2}{*}{$\$ 40$} & \multicolumn{2}{|c|}{ RCRA limits } \\
\hline & & & & & & $(\mathrm{mg} / \mathrm{L})$ & $(\mathbf{m g} / \mathbf{k g})$ \\
\hline \multicolumn{8}{|c|}{ Physical proparties and miscellaneous data } \\
\hline Density $(\mathrm{g} / \mathrm{mL})$ & & 1.008 & 1.013 & 1.013 & NA & & \\
\hline Depth (inches) & & 4 & 4 & 48 & NA & & \\
\hline Suspended solids ( $\mathrm{mg} / \mathrm{mL}$ ) & 0.1 & 0.6 & 1.1 & 0.9 & NA & & \\
\hline TDS $(\mathrm{mg} / \mathrm{mL})$ & 0.1 & 1,100 & 11.4 & 10.7 & NA & & \\
\hline $\operatorname{TOC}(\mathrm{L}=\mathrm{mg} / \mathrm{L}, \mathrm{S}=\mathrm{mg} / \mathrm{kg})$ & 1.0 & 12.1 & 1,120 & 1,310 & 28,000 & & \\
\hline TS $(\mathrm{L}=\mathrm{mg} / \mathrm{mL}, \mathrm{S}=\mathrm{mg} / \mathrm{g})$ & 0.1 & & 12.5 & 11.6 & 324 & & \\
\hline \multicolumn{8}{|c|}{$R C R A$ metals $(L=m g / L, S=m g / g g)$} \\
\hline $\mathrm{Ag}$ & 0.002 & $<0.002$ & $<0.002$ & 0.002 & 2.9 & 5.0 & 100 \\
\hline As & 0.40 & $<0.8$ & $<0.8$ & $<0.8$ & $<1$ & 5.0 & 100 \\
\hline $\mathrm{Ba}$ & 0.02 & 0.06 & $<0.04$ & $<0.04$ & 33 & 100 & 2,000 \\
\hline $\mathrm{Cd}$ & 0.01 & $<0.02$ & $<0.02$ & $<0.02$ & 6.6 & 1.0 & 20.0 \\
\hline $\mathrm{Cr}$ & 0.10 & $<0.1$ & 0.44 & $<0.1$ & $(180)$ & 5.0 & 100 \\
\hline $\mathrm{Hg}_{\mathrm{g}}$ & 0.005 & 0.1 & 0.1 & 0.15 & 70 & 0.2 & 4.0 \\
\hline $\mathrm{Ni}$ & 0.10 & $<0.2$ & $<0.2$ & $<0.2$ & 72 & NA & NA \\
\hline $\mathrm{Pb}$ & 0.50 & $<1$ & $<1$ & $<1$ & $(350)$ & 5.0 & 100 \\
\hline $\mathrm{Se}$ & 0.05 & $<0.09$ & $<0.09$ & $<0.09$ & $(<1)$ & 1.0 & 20.0 \\
\hline $\mathrm{T}$ & 0.05 & $<0.09$ & $<0.09$ & $<0.09$ & $<1$ & NA & NA \\
\hline \multicolumn{8}{|c|}{ Process metals $(L=m g / L, S=m g / k g)$} \\
\hline $\mathrm{Si}$ & 1.0 & 6.81 & 5.07 & 6.97 & NA & & \\
\hline $\mathrm{U}$ & 0.1 & 161 & 166 & 158 & 1,000 & & \\
\hline \multicolumn{8}{|c|}{ Anions $(L=m g / L, S=m g / k g)$} \\
\hline $\mathrm{Cl}^{-}$ & 1.0 & 12 & 12 & 12 & $4.9 \%$ & & \\
\hline $\mathrm{Cn}$ & 0.01 & 0.12 & 0.14 & 0.10 & - & & \\
\hline $\mathrm{F}$ & 1.0 & 1.0 & 1.0 & 1.0 & $<0.8 \%$ & & \\
\hline $\mathrm{NO}_{3}$ & 5.0 & 8.0 & 8.4 & 8.2 & $\bullet$ & & \\
\hline $\mathrm{PO}_{4}^{-2}$ & 5.0 & $<5$ & $<5$ & $<.5$ & • & & \\
\hline $\mathrm{SO}_{4}^{-2}$ & 5.0 & 21 & 22 & 22 & $14 \%$ & & \\
\hline \multicolumn{8}{|c|}{ Alkalinity } \\
\hline $\mathrm{CO}_{3}^{-2}(\mathrm{~mol})$ & 0.01 & 0.04 & 0.04 & 0.04 & * & & \\
\hline $\mathrm{HCO}_{3}^{\circ}(\mathrm{mol})$ & 0.01 & 0.09 & 0.08 & 0.09 & • & & \\
\hline $\mathrm{pH}$ (units) & 0.1 & 9.9 & 9.9 & 9.9 & NA & & \\
\hline \multicolumn{8}{|c|}{ Radiochanical screening ( $L=B q / m L, S=B q(g)$} \\
\hline Gross alpha & & $2.1 \mathrm{E}+02$ & $2.0 \mathrm{E}+02$ & $2.0 \mathrm{E}+02$ & $2.5 \mathrm{E}+05$ & & \\
\hline Gross beta & & $1.4 \mathrm{E}+0.5$ & $1.4 \mathrm{E}+05$ & $1.4 \mathrm{E}+05$ & $2.0 \mathrm{E}+07$ & & \\
\hline \multicolumn{8}{|c|}{ Betalganma anitters $(L=B q / m L, S=B q g)$} \\
\hline${ }^{137} \mathrm{Cs}$ & & $1.4 \mathrm{E}+05$ & $1.4 \mathrm{E}+05$ & $1.4 \mathrm{E}+05$ & $2.5 \mathrm{E}+05$ & & \\
\hline${ }^{14} \mathrm{C}$ & & $3.6 \mathrm{E}+02$ & $4.8 \mathrm{E}+02$ & $2.3 \mathrm{E}+02$ & $1.7 \mathrm{E}+01$ & & \\
\hline${ }^{152}$ Eu & & $\cdot$ & $\cdot$ & $\cdot$ & $3.8 \mathrm{E}+04$ & & \\
\hline${ }^{154} \mathrm{Eu}$ & & * & - & - & $2.6 \mathrm{E}+0.4$ & & \\
\hline${ }^{155} \mathrm{Eu}$ & & $*$ & + & $\cdot$ & $3.8 \mathrm{E}+03$ & & \\
\hline${ }^{3} \mathrm{H}$ & & $2.1 \mathrm{E}+02$ & $2.1 \mathrm{E}+02$ & $2.1 E+02$ & $9.5 \mathrm{E}+01$ & & \\
\hline${ }^{60} \mathrm{Co}$ & & $<75$ & $<75$ & $<75$ & $6.4 \mathrm{E}+04$ & & \\
\hline${ }^{40} \mathrm{Sr}$ & & $2.8 \mathrm{E}+03$ & $2.5 E+0.3$ & $2.7 E+03$ & $1.2 \mathrm{E}+07$ & & \\
\hline
\end{tabular}


Waste Characterization Data for ORNL Inactive LLLW Tanks

Rev. 0 / June 1992

Table 29.1. (continued)

\begin{tabular}{|c|c|c|c|c|c|c|}
\hline \multirow{2}{*}{ Parameter } & \multirow{2}{*}{$\begin{array}{c}D L \\
(m g / L)\end{array}$} & \multirow{2}{*}{ L112 } & \multirow{2}{*}{ L39 } & \multirow{2}{*}{$S 40$} & \multicolumn{2}{|c|}{ RCRA limits } \\
\hline & & & & & $(m g / L)$ & $(\mathrm{mg} / \mathrm{kg})$ \\
\hline \multicolumn{7}{|c|}{ Alpha amitters $(L=B q$ monl, $S=B q / g)$} \\
\hline${ }^{233} \mathrm{U}$ & & $1.8 \mathrm{E}+02$ & $1.8 E+02$ & $8.3 E+03$ & & \\
\hline${ }^{238} \mathrm{Pu}$ & & $\cdot$ & - & $3.1 E+03$ & & \\
\hline${ }^{239} \mathrm{Pu}$ & & - & - & $5.1 \mathrm{E}+03$ & & \\
\hline${ }^{244} \mathrm{Cm}$ & & - & - & $<200$ & & \\
\hline${ }^{252} \mathrm{Cf}$ & & - & - & $1.8 \mathrm{E}+05$ & & \\
\hline
\end{tabular}

Table 29.2 Organic analytical data hits for tank T-2

\begin{tabular}{|c|c|c|c|c|c|c|c|}
\hline Parameter & $\begin{array}{l}\text { CRQL } \\
(\mu g / L)\end{array}$ & L38 & L.39 & L112 & S40 & $\begin{array}{c}\text { RCRA limit } \\
(\mu g / L)\end{array}$ & $\begin{array}{l}\text { CRQL } \\
(\mu g / k g)\end{array}$ \\
\hline \multicolumn{8}{|c|}{ Volatile onganic componund analywis $(L=4 g / L, S=n g / h)$} \\
\hline Acetone & 10 & $20 \mathrm{~B}$ & $22 \mathrm{~B}$ & $17 \mathrm{~B}$ & & & \\
\hline Benzene & 5 & $3 \mathrm{~J}$ & & & & 500 & \\
\hline Chloroform & 5 & $340 \mathrm{BE}$ & $370 \mathrm{BE}$ & $310 \mathrm{BE}$ & & 6000 & \\
\hline Methylene chloride & 5 & $1000 \mathrm{BE}$ & $820 \mathrm{BE}$ & $860 \mathrm{BE}$ & & & \\
\hline Toluene & 5 & $12 \mathrm{~B}$ & $7 \mathrm{~B}$ & $8 \mathrm{~B}$ & & & \\
\hline \multicolumn{8}{|c|}{ Semivolatile compoumd anabuis $(L=\mu g / L, S=\mu g / k g)$} \\
\hline 2,4,5-Trichlorophenol & 10 & $99 \mathrm{~J}$ & $110 \mathrm{~J}$ & $120 \mathrm{~J}$ & & & 330 \\
\hline 2,4-Dichlorophenol & 10 & $100 \mathrm{~J}$ & $140 \mathrm{~J}$ & & & & 330 \\
\hline 2-Nitrophenol & 10 & $170 \mathrm{~J}$ & $200 \mathrm{~J}$ & $180 \mathrm{~J}$ & & & 330 \\
\hline Bis(2-ethylhexyl)phthalate & 10 & $200 \mathrm{~J}$ & $300 \mathrm{~J}$ & $280 \mathrm{~J}$ & 24,000 & & 330 \\
\hline Di-n-butylphthalate & 10 & & & $24 \mathrm{~J}$ & $4600 \mathrm{~J}$ & & 330 \\
\hline Diethylphthalate & 10 & & & & $3800 \mathrm{~J}$ & & 330 \\
\hline Naphthalene & 10 & & & & $3300 \mathrm{~J}$ & & 330 \\
\hline
\end{tabular}

$\overline{\mathrm{CRQL}}=$ contract required quantitation limit 


\section{TANK T-3}

\subsection{Tank Description}

Tank T-3 is a 25,000-gal carbon steel tank located in Melton Valley at the OHF. The underground tank was installed at the site in 1963 to store LLLW for mixing into grout for waste injection by hydrofracture.

\subsubsection{Characterization Summary}

\subsection{Aqueous liquid}

The results from Tank T-3 are shown in Tables 2.10.1 and 2.10.2. Very little organic matter was detected in the samples from this tank. The major organic compounds were determined by DAI-GC, and consisted of acetone $(4 \mathrm{mg} / \mathrm{L})$, methanol $(28 \mathrm{mg} / \mathrm{L})$, and $\mathrm{n}$-butyl alcohol $(80 \mathrm{mg} / \mathrm{L})$. As noted in Sect. 2 , these identifications cannot be considered absolute because the method does not include MS confirmation. However, where the constituent was also identified by VOA, there is more confidence in the DAI-GC result. Of the CLP TCL constituents, only acetone was detected at $180 \mu \mathrm{g} / \mathrm{L}$ by the purge-and-trap GC-MS VOA. The value for the VOA being lower than the value for DAI-GC is probably because of low purging efficiency at high concentration levels.

Volatile TICs were insignificant. Only benzyl alcohol was estimated at $100 \mu \mathrm{g} / \mathrm{L}$ in the aqueous liquid SVOA. Among the TICs were dibromonitrophenol at $2000 \mu \mathrm{g} / \mathrm{L}$ and TBP at $2000 \mu \mathrm{g} / \mathrm{L}$. Both of these TICs were found in other samples as well. The presence of a nitrated phenol suggests nitration reactions in the tank. The brominated product was unexpected, but TBP has been used in large amounts at ORNL, and its presence was expected.

The SVOA phenol and 2-fluorophenol surrogate standards were not recovered. This may reflect the highly alkaline nature of the samples (the $\mathrm{pH}$ of $\mathrm{T}-3 / \mathrm{L}-042$ was 12.7 ).

\subsubsection{Sludge}

Two phthalates, diethyl- and di-n-butylphthalate, were detected at 2 and $3 \mathrm{mg} / \mathrm{kg}$, respectively. It is not clear why neither dibromonitrophenol nor TBP was detected in the sludge SVOA TICs, unless the analysis was not sufficiently sensitive. 
Waste Characterization Data for ORNL Inactive LLLW Tanks Rev. 0 / June 1992

Table 2.10.1. Analytical data for tank T-3

\begin{tabular}{|c|c|c|c|c|c|}
\hline \multirow{2}{*}{ Parameter } & \multirow{2}{*}{$\underset{(\mathrm{mg} / \mathrm{L})}{\mathrm{DL}}$} & \multirow{2}{*}{ L42 } & \multirow{2}{*}{$\$ 43$} & \multicolumn{2}{|c|}{ RCRA limits } \\
\hline & & & & $(\mathrm{mg} / \mathrm{L})$ & $(\mathrm{mg} / \mathrm{kg})$ \\
\hline \multicolumn{6}{|c|}{ Physical properties and miscellaneous data } \\
\hline Density $(g / m L)$ & & 1.035 & 1.930 & & \\
\hline Depth (inches) & & 5 & NA & & \\
\hline Suspended solids (mg/mL) & 0.1 & 1.5 & NA & & \\
\hline $\mathrm{IDS}(\mathrm{mg} / \mathrm{mL})$ & 0.1 & 51.9 & NA & & \\
\hline $\mathrm{TOC}(\mathrm{L}=\mathrm{mg} / \mathrm{L}, \mathrm{S}=\mathrm{mg} / \mathrm{kg})$ & 1.0 & 12,600 & 9,140 & & \\
\hline $\mathrm{TS}(\mathrm{L}=\mathrm{mg} / \mathrm{mL}, \mathrm{S}=\mathrm{mg} / \mathrm{g})$ & 0.1 & 53.4 & 364 & & \\
\hline \multicolumn{6}{|c|}{$R C R A$ metals $(L=m g / L,<0.01 S=m g / k g)$} \\
\hline Ag & 0.002 & $<0.01$ & 0.15 & 5.0 & 100 \\
\hline As & 0.40 & 0.4 & $<3$ & 5.0 & 100 \\
\hline $\mathbf{B a}$ & 0.02 & $<0.02$ & 76 & 100 & 2,000 \\
\hline Cd & 0.01 & $<0.01$ & 8.5 & 1.0 & 20.0 \\
\hline $\mathrm{Cr}$ & 0.10 & 14 & $(69)$ & 5.0 & 100 \\
\hline $\mathrm{Hg}$ & 0.005 & 5.7 & 40 & 0.2 & 4.0 \\
\hline $\mathrm{Ni}$ & 0.10 & $<0.1$ & 57 & NA & NA \\
\hline $\mathrm{Pb}$ & 0.50 & $<0.5$ & $(300)$ & 5.0 & 100 \\
\hline Se & 0.05 & $<0.5$ & $(0.74)$ & 1.0 & 20.0 \\
\hline $\mathrm{T} 1$ & 0.05 & $<0.5$ & $<0.6$ & NA & NA \\
\hline \multicolumn{6}{|c|}{ Proceas metals $(L=m g / L, S=m g / k g)$} \\
\hline $\mathbf{S i}$ & 1.0 & 77.1 & NA & & \\
\hline $\mathrm{U}$ & 0.1 & 0.2 & 3060 & & \\
\hline \multicolumn{6}{|c|}{ Anions $(L=m g / L, S=m g / k g)$} \\
\hline $\mathrm{Cl}^{-}$ & 1.0 & 7.0 & $29 \%$ & & \\
\hline $\mathrm{Cn}$ & 0.01 & 1.10 & 0.20 & & \\
\hline $\mathrm{F}$ & 1.0 & 1.8 & $<12.7 \%$ & & \\
\hline $\mathrm{NO}_{3}^{-}$ & 5.0 & 53 & * & & \\
\hline $\mathrm{PO}_{4}^{-2}$ & 5.0 & 160 & $<62.5 \%$ & & \\
\hline $\mathrm{SO}_{4}^{-2}$ & 5.0 & 15 & $<62.5 \%$ & & \\
\hline \multicolumn{6}{|c|}{ Alkalinity } \\
\hline $\mathrm{CO}_{3}^{-2}(\mathrm{~mol})$ & 0.01 & 0.08 & - & & \\
\hline $\mathrm{OH}^{\circ}(\mathrm{mol})$ & 0.01 & 0.03 & - & & \\
\hline $\mathrm{pH}$ (units) & 0.1 & 12.7 & NA & & \\
\hline \multicolumn{6}{|c|}{ Radiochemical screening ( $L=B q / m L, S=B q / g)$} \\
\hline Gross alpha & & $2.0 \mathrm{E}+00$ & $2.0 \mathrm{E}+05$ & & \\
\hline Gross beta & & $2.8 \mathrm{E}+05$ & $2.5 E+07$ & & \\
\hline \multicolumn{6}{|c|}{ Beta/garnma emitters ( $L=1 \mathrm{Rq} / \mathrm{m} L, S=B q / \mathrm{g})$} \\
\hline${ }^{137} \mathrm{Cs}$ & & $2.7 \mathrm{E}+05$ & $1.3 \mathrm{E}+06$ & & \\
\hline${ }^{14} \mathrm{C}$ & & $\cdot$ & $7.6 \mathrm{E}+02$ & & \\
\hline${ }^{152} \mathrm{Eu}$ & & • & $5.1 \mathrm{E}+04$ & & \\
\hline${ }^{154} \mathrm{Eu}$ & & * & $5.3 \mathrm{E}+04$ & & \\
\hline${ }^{3} \mathrm{H}$ & & $1.7 \mathrm{E}+02$ & $7.7 E+01$ & & \\
\hline${ }^{60} \mathrm{Co}$ & & $3.6 \mathrm{E}+02$ & $1.6 \mathrm{E}+05$ & & \\
\hline${ }^{90} \mathrm{Sr}$ & & $3.0 \mathrm{E}+02$ & $8.1 \mathrm{E}+06$ & & \\
\hline
\end{tabular}


Waste Characterization Data for ORNL Inactive LLLW Tanks

Table 2.10 .1 (continued)

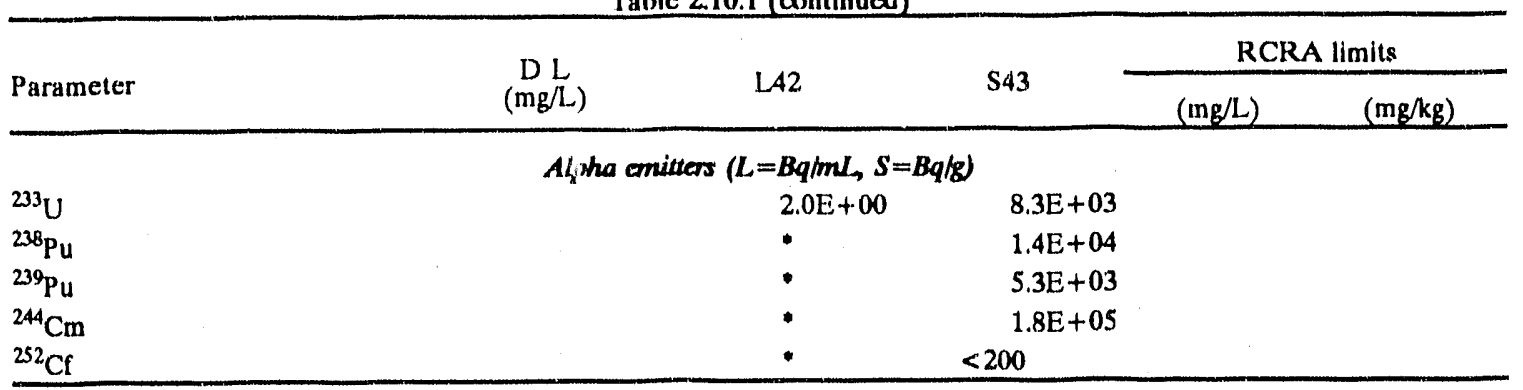

$\mathrm{DL}=$ Detection limit

Table 2.10.2. Organic analytical data hits for tank T-3

\begin{tabular}{|c|c|c|c|c|c|}
\hline Parameter & $\begin{array}{l}\text { CRQL } \\
(\mu g / L)\end{array}$ & LA2 & $S 43$ & $\begin{array}{c}\text { RCRA limit } \\
(\mu \mathrm{g} / \mathrm{L})\end{array}$ & $\begin{array}{l}\text { CRQL } \\
\left(\mu_{\mathrm{g}} / \mathrm{kg}\right)\end{array}$ \\
\hline \multicolumn{6}{|c|}{$D A I-G C$ analysis $(L=k g / L, S=n g / / g g)$} \\
\hline Acetone & & 4,000 & & & \\
\hline Methyl alcohol & & 28,000 & & & \\
\hline N-Butyl alcohol & & 30,000 & & & \\
\hline \multicolumn{6}{|c|}{ Volatile orgunic compound analysis ( $L=m g / L, S=\mu g / \mathrm{kg})$} \\
\hline Acetone & 10 & $180 \mathrm{~B}$ & & & \\
\hline Chloroform & 5 & $3 \mathrm{JB}$ & & 6,000 & \\
\hline Methylene chloride & 5 & $3 \mathrm{JB}$ & & & \\
\hline Toluene & 5 & $9 \mathrm{~B}$ & & & \\
\hline \multicolumn{6}{|c|}{ Semivolatile compound analywis ( $L=\mu g / L, S=\mu g / k g)$} \\
\hline Benzyl alcohol & 10 & $120 \mathrm{~J}$ & & & 330 \\
\hline Di-n-butylphthalate & 10 & & $3,100 \mathrm{~J}$ & & 330 \\
\hline Diethylphthalate & 10 & & $2,000 \mathrm{~J}$ & & 330 \\
\hline
\end{tabular}

CRQL $=$ contract required quantitation limit 


\section{TANK T-4}

\subsection{Tank Description}

Tank T-4 is a 25,000-gal carbon steel tank located in Melton Valley at the OHF. The underground tank was installed at the site in 1963 to store LLLW for mixing into grout for waste injection by hydrofracture.

\subsubsection{Characterization Summary}

\subsubsection{Aqueous liquid}

The results from tank T-4 are shown in Tables 2.11.1 and 2.11.2. The three aqueous liquid samples for tank T-4 yielded very similar results, as shown in Table 2.11.2. DAI-GC showed concentrations exceeding $1000 \mu \mathrm{g} / \mathrm{L}$ each of acetone, ethyl alcohol, isopropyl alcohol, methanol, and n-butyl alcohol. VOA confirmed the presence of acetone and also indicated chloroform. Other TCL volatile organics were present at very low levels.

Volatile TICs were low in concentration; the major one was heptanone $(80 \mu \mathrm{g} / \mathrm{L}$, found in L44). No semivolatile TCL organic compounds were detected in the liquids. Major SVOA TICs were methylhexanone $(800 \mu \mathrm{g} / \mathrm{L}$ in L45) and TBP (3000 $\mu \mathrm{g} / \mathrm{L}$ in L111).

\subsubsection{Sludge}

The sludge contained only the ubiquitous phthalates.

Two of the neutral and two of the acid surrogate standard recoveries were low for the sludge. Low recoveries of acidic surrogate standards from the alkaline sludges were often encountered, suggesting a need for $\mathrm{pH}$ adjustment currently unspecified in EPA method 3550. Studies are under way to improve the surrogate standard recoveries in such sample matrices.

The samples for tanks T-1, T-2, and T-9 were each prepared as a group. 
Waste Characterization Data for ORNL Inactive LLLW Tanks Rev. 0 / June 1992

Table 2.11.1. Analytical Data for Tank T-4

\begin{tabular}{|c|c|c|c|c|c|c|c|}
\hline \multirow{2}{*}{ Parameter } & \multirow{2}{*}{$\begin{array}{c}D L \\
(\mathrm{mg} / \mathrm{L})\end{array}$} & \multirow{2}{*}{ L44 } & \multirow{2}{*}{ LAS } & \multirow{2}{*}{ S46 } & \multirow{2}{*}{ L111 } & \multicolumn{2}{|c|}{ RCRA limits } \\
\hline & & & & & & \multicolumn{2}{|c|}{$(\mathrm{mg} / \mathrm{L})(\mathrm{mg} / \mathrm{kg})$} \\
\hline \multicolumn{8}{|c|}{ Physical properties and miscellaneous data } \\
\hline Density $(g / m L)$ & & 1.020 & 1.016 & 1.235 & 1.016 & & \\
\hline Depth (inches) & & 4 & 34 & NA & 0 & & \\
\hline Suspended solids(mg/mL) & 0.1 & 2.7 & $<0.1$ & NA & 0.3 & & \\
\hline $\mathrm{TDS}(\mathrm{mg} / \mathrm{mL})$ & 0.1 & 23.6 & 23.7 & NA & 23.6 & & \\
\hline $\operatorname{TOC}(\mathrm{L}=\mathrm{mg} / \mathrm{L}, \mathrm{S}=\mathrm{mg} / \mathrm{kg})$ & 1.0 & 460 & 473 & 4620 & 460 & & \\
\hline TS $(\mathrm{L}=\mathrm{mg} / \mathrm{mL}, \mathrm{S}=\mathrm{mg} / \mathrm{g})$ & 0.1 & 26.3 & 23.4 & 253 & 23.9 & & \\
\hline \multicolumn{8}{|c|}{$R C R A$ metals $(L=m g / L, S=m g / k g)$} \\
\hline$A g$ & 0.002 & 0.017 & 0.02 & (1.7) & 0.018 & 5 & 100 \\
\hline As & 0.40 & $<0.8$ & $<0.8$ & $<4$ & $<0.4$ & $s$ & 100 \\
\hline $\mathrm{Ba}$ & 0.02 & $<0.04$ & $<0,04$ & $<50$ & $<0.02$ & 100 & 2000 \\
\hline $\mathrm{Cd}$ & 0.01 & $<0.02$ & $<0,02$ & 10 & $<0.01$ & 1 & 20 \\
\hline $\mathrm{Cr}$ & 0.10 & 9.4 & 14 & (102) & 13 & 5 & 100 \\
\hline $\mathrm{Hg}$ & 0.005 & 1.1 & 2.7 & 585 & 7.9 & 0.2 & 4.0 \\
\hline $\mathrm{Ni}$ & 0.10 & $<0.2$ & $<0.2$ & 160 & $<0.1$ & NA & NA \\
\hline $\mathrm{Pb}$ & 0.50 & $<1$ & $<1$ & $(510)$ & $<0.5$ & 5 & 100 \\
\hline $\mathrm{Se}$ & 0.05 & $<0.09$ & $<0.09$ & $(1.5)$ & $<0.23$ & 1 & 20 \\
\hline $\mathrm{Tl}$ & 0.05 & $<0.09$ & $<0.09$ & 0.73 & $<0.23$ & NA & NA \\
\hline \multicolumn{8}{|c|}{ Process metals $(L=m g / L, S=m g / k g)$} \\
\hline $\mathrm{Si}$ & 1.0 & 1.96 & 1.45 & NA & 1.96 & & \\
\hline $\mathrm{U}$ & 0.1 & 25.7 & 27.8 & 1850 & 23.3 & & \\
\hline \multicolumn{8}{|c|}{ Anions $(L=m g / L, S=m g / k g)$} \\
\hline $\mathrm{Cl}^{\cdot}$ & 1.0 & 3.1 & 3.4 & $<0.83 \%$ & 3.4 & & \\
\hline $\mathrm{Cn}$ & 0.01 & 0.54 & 0.59 & $\cdot$ & $\cdot$ & & \\
\hline$F$ & 1.0 & 1.1 & 1.3 & $<0.83 \%$ & 1.1 & & \\
\hline $\mathrm{NO}_{3}^{-}$ & 5.0 & 26 & 25 & $\cdot$ & 26 & & \\
\hline $\mathrm{PO}_{4}^{-2}$ & 5.0 & 51 & 50 & $<42 \%$ & 50 & & \\
\hline $\mathrm{SO}_{4}^{-2}$ & 5.0 & 6.3 & 5.9 & $<42 \%$ & 6.3 & & \\
\hline \multicolumn{8}{|c|}{ Alkalinity } \\
\hline $\mathrm{CO}_{3}^{-2}(\mathrm{~mol})$ & 0.01 & 0.05 & 0.05 & - & 0.05 & & \\
\hline $\mathrm{OH}^{\circ}(\mathrm{mol})$ & 0.01 & 0.01 & 0.01 & - & 0.01 & & \\
\hline $\mathrm{pH}$ (units) & 0.01 & 11.7 & 11.7 & NA & 11.7 & & \\
\hline \multicolumn{8}{|c|}{ Radiochemical screening ( $L=B q / m L, S=B q / g)$} \\
\hline Gross alpha & & $3.6 \mathrm{E}+01$ & $4.9 \mathrm{E}+01$ & $3.7 \mathrm{E}+05$ & $3.8 \mathrm{E}+01$ & & \\
\hline Gross beta & & $2.8 \mathrm{E}+05$ & $3.1 E+05$ & $3.6 \mathrm{E}+07$ & $3.1 \mathrm{E}+05$ & & \\
\hline \multicolumn{8}{|c|}{ Besa/gramma amitters $(L=B q / m L, S=B q / g)$} \\
\hline${ }^{137} \mathrm{Cs}$ & & $3.0 \mathrm{E}+05$ & $3.0 \mathrm{E}+05$ & $4.5 \mathrm{E}+05$ & $3.0 \mathrm{E}+05$ & & \\
\hline${ }^{14} \mathrm{C}$ & & 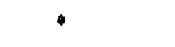 & - & $5.1 \mathrm{E}+02$ & $*$ & & \\
\hline${ }^{152} \mathrm{Eu}$ & & • & - & $5.2 \mathrm{E}+0.4$ & - & & \\
\hline
\end{tabular}


Waste Characterization Data for ORNL Inactive LLLW Tanks Rev. 0 / June 1992

Table 2.11 .1 (continued)

\begin{tabular}{|c|c|c|c|c|c|c|}
\hline \multirow{2}{*}{ Parameter } & \multirow{2}{*}{$\begin{array}{c}\text { D L } \\
(\mathrm{mg} / \mathrm{L})\end{array}$} & \multirow{2}{*}{ LA4 } & \multirow{2}{*}{ L45 } & \multirow{2}{*}{ S46 } & \multirow{2}{*}{ L111 } & \multirow{2}{*}{$\frac{\text { RCRA limits }}{(\mathrm{mg} / \mathrm{l})(\mathrm{mg} / \mathrm{kg})}$} \\
\hline & & & & & & \\
\hline \multicolumn{7}{|c|}{ Betalgamma emitters ( $L=B q$ hrn $L, S=B q / g)$ (continued) } \\
\hline${ }^{154} \mathrm{Eu}$ & & $*$ & $*$ & $4.4 \mathrm{E}+04$ & * & \\
\hline${ }^{155} \mathrm{Eu}$ & & $\cdot$ & $\cdot$ & $7.0 \mathrm{E}+03$ & * & \\
\hline${ }^{3} \mathrm{H}$ & · & $1.1 \mathrm{E}+02$ & $1.1 \mathrm{E}+02$ & $2.8 \mathrm{E}+01$ & $1.1 \mathrm{E}+02$ & \\
\hline${ }^{60} \mathrm{Co}$ & & $6.4 \mathrm{E}+01$ & $5.2 \mathrm{E}+01$ & $6.0 \mathrm{E}+04$ & $5.2 \mathrm{E}+01$ & \\
\hline${ }^{90} \mathrm{Sr}$ & & $1.2 \mathrm{E}+03$ & $1.4 \mathrm{E}+03$ & $2.2 \mathrm{E}+07$ & $1.4 \mathrm{E}+03$ & \\
\hline \multicolumn{7}{|c|}{ Alphe envitters $(L=B q$ ton $L, S=B q / g)$} \\
\hline${ }^{233} \mathrm{U}$ & & $2.2 \mathrm{E}+01$ & $2.9 \mathrm{E}+01$ & $7.1 \mathrm{E}+03$ & $2.3 \mathrm{E}+01$ & \\
\hline${ }^{238} \mathrm{Pu}$ & & - & - & $2.2 \mathrm{E}+04$ & $\cdot$ & \\
\hline${ }^{238} \mathrm{Th} /{ }^{232} \mathrm{Th}$ & & $"$ & - & $5.8 \mathrm{E}+02$ & - & \\
\hline${ }^{238} \mathrm{U}$ & & • & - & $\cdot$ & - & \\
\hline${ }^{239} \mathrm{Pu}$ & & - & * & $4.6 \mathrm{E}+03$ & • & \\
\hline${ }^{241} \mathrm{Am}$ & & • & - & $8.2 E+03$ & • & \\
\hline${ }^{244} \mathrm{Cm}$ & & - & $\cdot$ & $2.1 \mathrm{E}+05$ & - & \\
\hline
\end{tabular}

Table 211.2. Organic analytical data hits for tank $T-4$

\begin{tabular}{|c|c|c|c|c|c|c|c|}
\hline Parameter & $\begin{array}{l}\text { CRQL } \\
(\mu \mathrm{g} / \mathrm{L})\end{array}$ & L44 & LAS & S46 & L111 & $\begin{array}{c}\text { RCRA } \\
\text { limit } \\
(\mu \mathrm{g} / \mathrm{L})\end{array}$ & $\begin{array}{l}\text { CRQL } \\
(\mu \mathrm{g} / \mathrm{kg})\end{array}$ \\
\hline \multicolumn{8}{|c|}{$D A I-G C$ anaburis $(L=\mu g / L, S=\mu g / \mathrm{gg})$} \\
\hline Acetone & & 7,000 & 8,000 & & 7,000 & & \\
\hline Ethyl alcohol & & 37,000 & 37,000 & & 37,000 & & \\
\hline 1-Propyl alcohol & & 3,000 & 3,000 & & 3,000 & & \\
\hline Methyl aicohol & & 46,000 & 27,000 & & 42,000 & & \\
\hline N-Butyl & & 3,000 & 6,000 & & 3,000 & & \\
\hline \multicolumn{8}{|c|}{ Volatile organic compound analywir $(L=m / L, S=$ mg/kg) } \\
\hline 2-Hexanone & 10 & & 20 & & & & \\
\hline 4-Methyl-2-pentanone & 10 & 11 & 10 & & & & \\
\hline Acetone & 10 & $400 \mathrm{BE}$ & $220 \mathrm{BE}$ & & 72, B & & \\
\hline Benzene & 5 & 7 & 10 & & 7 & 500 & \\
\hline Bromodichloromethane & 5 & $7 \mathrm{~B}$ & & & & & \\
\hline Chlorobenzene & 5 & & $4 \mathrm{~J}$ & & & 100,000 & \\
\hline Chloroform & 5 & $170 \mathrm{~B}$ & $160 \mathrm{~B}$ & & $120 \mathrm{~B}$ & 6,000 & \\
\hline Ethylbenzene & 5 & 20 & 20 & & 5 & & \\
\hline Methylene chloride & 5 & $12 \mathrm{~B}$ & $14 \mathrm{~B}$ & & $12 \mathrm{~B}$ & & \\
\hline Toluene & 5 & $78 \mathrm{~B}$ & $99 \mathrm{~B}$ & & $60 \mathrm{~B}$ & & \\
\hline Trichloroethene & 5 & & $3 \mathrm{~J}$ & & & 500 & \\
\hline Xylene (total) & 5 & & 170 & & 36 & & \\
\hline \multicolumn{8}{|c|}{ Semivolatile compound analysis $(L=\mu g / L, S=\mu g / \mathrm{kg})$} \\
\hline Di-n-butylphthalate & & & & $3,400 \mathrm{~J}$ & & & 330 \\
\hline Diethylphthalate & & & & $2,400 \mathrm{~J}$ & & & 330 \\
\hline
\end{tabular}

$\overline{C R Q L}=$ contract required quantitation limit 


\section{TANK T-9}

\subsection{Tank Description}

Tank T-9 is a 25,000-gal carbon steel tank located in the Melton Valley at the OHF. The underground tank was installed at the site in 1963 to store LLLW for mixing into grout for waste injection by hydrofracture.

\subsection{Characterization Summary}

\section{Aqueous liquid}

The results for Tank T-9 are shown in Tables 2.12.1 and 2.12.2. Tank T-9 did not contain appreciable concentrations of either volatile or semivolatile organic compounds, except for $17 \mathrm{mg} / \mathrm{L}$ of methanol determined by DAI-GC. Sample LA7 contained $400 \mu \mathrm{g} / \mathrm{L}$ of benzoic acid and $200 \mu \mathrm{g} / \mathrm{L}$ of benzyl alcohol. TBP at $30 \mathrm{mg} / \mathrm{L}$ was the main TIC.

\section{Sludge}

Only phthalates were determined as TCL constituents in the sludge; TBP was determined as a TIC at $2 \mathrm{mg} / \mathrm{kg}$.

Samples from tanks TH-2, TH-3, and W-11 were each processed together. 
Waste Characterization Data for ORNL Inactive LLLW Tanks

Rev. 0/June 1992

Section $2.12 /$ page 2 of 3

Table 2.12.1. Analytical data for tank T-9

\begin{tabular}{|c|c|c|c|c|c|}
\hline \multirow{2}{*}{ Parameter } & \multirow{2}{*}{$\frac{D L}{(m g / L)}$} & \multirow{2}{*}{$\llcorner 47$} & \multirow{2}{*}{ L48 } & \multicolumn{2}{|c|}{ RCRA limits } \\
\hline & & & & $(\mathrm{mg} / \mathrm{L})$ & $(\mathrm{mg} / \mathrm{kg})$ \\
\hline \multicolumn{6}{|c|}{ Phossical properties and miscellaneous daea } \\
\hline Density (g/mL) & & 10.34 & NA & & \\
\hline Depth (inches) & & 6.5 & NA & & \\
\hline Suspended solids ( $\mathrm{mg} / \mathrm{mL})$ & 0.1 & 0.7 & NA & & \\
\hline $\mathrm{TDS}(\mathrm{mg} / \mathrm{mL})$ & 0.1 & 41.5 & N.A & & \\
\hline TOC $(\mathrm{L}=m g / \mathrm{L}, \mathrm{S}=\mathrm{mg} / \mathrm{kg})$ & 1.0 & 850 & 7620 & & \\
\hline TS $(L=m g / m L, ~ S=m g / g)$ & 0.1 & 42.2 & 343 & & \\
\hline \multicolumn{6}{|c|}{$R C R A$ metals $(L=m g / L, S=m g / k g)$} \\
\hline$A g$ & 0.002 & 0.01 & 0.21 & 5 & 100 \\
\hline As & 0.40 & $<0.8$ & $<2$ & 5 & 100 \\
\hline $\mathrm{Ba}$ & 0.02 & 0.12 & 115 & 100 & 2000 \\
\hline $\mathrm{Cd}$ & 0.01 & $<0.02$ & 7.8 & 1 & 20 \\
\hline $\mathrm{Cr}$ & 0.10 & 0.4 & $(<10)$ & 5 & 100 \\
\hline $\mathrm{Hg}_{\mathrm{g}}$ & 0.005 & 3.4 & 39 & 0.2 & 4 \\
\hline $\mathrm{Ni}$ & 0.10 & $<0.2$ & 390 & NA & NA \\
\hline $\mathrm{Pb}$ & 0.50 & $<1$ & $(540)$ & 5 & 100 \\
\hline Se & 0.05 & $<0.09$ & $(<2)$ & 1 & 20 \\
\hline $\mathrm{TI}$ & 0.05 & $<0.09$ & $<2$ & NA & NA \\
\hline \multicolumn{6}{|c|}{ Process mexals $(L=m g / h, s=m g / k g)$} \\
\hline $\mathrm{Si}$ & 1.0 & 9.76 & NA & & \\
\hline $\mathrm{U}$ & 0.1 & 8.52 & 29.30 & & \\
\hline \multicolumn{6}{|c|}{ Arions ( $L=m g / L, S=m g / k g)$} \\
\hline $\mathrm{Cl}^{-}$ & 1.0 & 290 & $3 \%$ & & \\
\hline$F$ & 1.0 & 1.0 & $<0.7 \%$ & & \\
\hline $\mathrm{NO}_{3}^{-}$ & 5.0 & 110 & $\cdot$ & & \\
\hline $\mathrm{PO}_{4}^{-2}$ & 5.0 & $<5$ & - & & \\
\hline $\mathrm{SO}_{4}^{-2}$ & 5.0 & 37 & $6.8 \%$ & & \\
\hline \multicolumn{6}{|c|}{ Alkaliniry } \\
\hline $\mathrm{CO}_{3}^{-2}(\mathrm{~mol})$ & 0.01 & $<0.01$ & - & & \\
\hline $\mathrm{HCO}_{3}^{\circ}(\mathrm{mol})$ & 0.01 & 0.03 & $\bullet$ & & \\
\hline $\mathrm{pH}$ (units) & 0.1 & 9.1 & NA & & \\
\hline \multicolumn{6}{|c|}{ Radiochemical screening ( $L=B q / m L, S=B q / g)$} \\
\hline Gross alpha & & $7.0 \mathrm{E}+02$ & $1.5 E+05$ & & \\
\hline Grass beta & & $3.4 \mathrm{E}+05$ & $1.6 \mathrm{E}+07$ & & \\
\hline \multicolumn{6}{|c|}{ Besalgarmune emitters $\left(L=B q / m L_{2}, S=B q / g\right)$} \\
\hline${ }^{137} \mathrm{Cs}$ & & $2.9 E+05$ & $4.0 \mathrm{E}+05$ & & \\
\hline${ }^{14} \mathrm{C}$ & & $\cdot$ & $2.2 \mathrm{E}+03$ & & \\
\hline${ }^{152} \mathrm{Eu}$ & & • & $3.5 E+04$ & & \\
\hline${ }^{154} \mathrm{Eu}$ & & $\cdot$ & $8.9 \mathrm{E}+03$ & & \\
\hline${ }^{3} \mathrm{H}$ & & $1.6 \mathrm{E}+02$ & $3.4 \mathrm{E}+01$ & & \\
\hline${ }^{60} \mathrm{Co}$ & & $6.0 \mathrm{E}-03$ & $4.3 E+04$ & & \\
\hline${ }^{90} \mathrm{Sr}$ & & $3.6 \mathrm{E}+1.04$ & $1.4 \mathrm{E}+07$ & & \\
\hline
\end{tabular}


Waste Characterization Data for ORNL Inactive LLLW Tanks

Table 212.1 (oontinued)

\begin{tabular}{|c|c|c|c|c|c|}
\hline \multirow{2}{*}{ Parameter } & \multirow{2}{*}{$\begin{array}{c}D L \\
(\mathrm{mg} / \mathrm{L})\end{array}$} & \multirow{2}{*}{$\llcorner 47$} & \multirow{2}{*}{ LA8 } & \multicolumn{2}{|c|}{ RCRA limits } \\
\hline & & & & $(m g /)$. & $(\mathrm{mg} / \mathrm{kg})$ \\
\hline \multicolumn{6}{|c|}{ Alpha emittess $(L=B q / m L, S=B q / g)$} \\
\hline${ }^{233} \mathrm{U}$ & & $6.6 \mathrm{E}+02$ & $4.4 \mathrm{E}+03$ & & \\
\hline${ }^{236} \mathrm{Pu}$ & & $\cdot$ & $1.0 \mathrm{E}+04$ & & \\
\hline${ }^{239} \mathrm{pu}$ & & - & $4.3 E+03$ & & \\
\hline${ }^{244} \mathrm{Cm}$ & & - & $9.7 \mathrm{E}+04$ & & \\
\hline${ }^{252} \mathrm{Cf}$ & & - & $<2$ & & \\
\hline
\end{tabular}

$\mathrm{DL}=$ Detection limit

Table 212.2. Organic analytical data hils for tank T-9

\begin{tabular}{|c|c|c|c|c|c|}
\hline Parameter & $\begin{array}{l}\text { CRQL } \\
(\mu g / L)\end{array}$ & LA7 & S48 & $\begin{array}{c}\text { RCRA limit } \\
(\mu g / L)\end{array}$ & $\begin{array}{l}\text { CRQL } \\
(\mu g / k g)\end{array}$ \\
\hline \multicolumn{6}{|c|}{$D A J-G C$ arahusis $(L=\mu g / L, S=\mu g / k g)$} \\
\hline Methyl alcohol & & 7,000 & & & \\
\hline \multicolumn{6}{|c|}{ Volatile organic compround anabuis ( $L=n g / L, S=$ ng/kg) } \\
\hline 1,1-Dichlorothene & 5 & $2 \mathrm{~J}$ & & 700 & \\
\hline Acetone & 10 & $23 \mathrm{~B}$ & & & \\
\hline Benzene & 5 & 11 & & 500 & \\
\hline Carbon disulfide & 5 & $4 \mathrm{~J}$ & & & \\
\hline Chlorobenzene & 5 & 6 & & 100,000 & \\
\hline Chloroform & 5 & $13 \mathrm{~B}$ & & 6,000 & \\
\hline Methylene chloride & 5 & $600 \mathrm{BE}$ & & & \\
\hline Toluene & 5 & $19 \mathrm{~B}$ & & & \\
\hline Trichloroethene & 5 & $5 \mathrm{~J}$ & & 500 & \\
\hline \multicolumn{6}{|c|}{ Senivolatile compound anabsis ( $L=\mu g / L, S=$ me/kg) } \\
\hline Benzoic acid & 50 & $340 \mathrm{~J}$ & & & \\
\hline Benzyl alcohol & 10 & $160 \mathrm{~J}$ & & & \\
\hline Di-n-butylphthalate & 10 & & $3,700 \mathrm{~J}$ & & 330 \\
\hline Diethylphthalate & 10 & & $3,000 \mathrm{~J}$ & & 330 \\
\hline
\end{tabular}

$\overline{C R Q L}=$ contract required quantitation limit 


\subsection{TANK TH-1}

\subsubsection{Tank Description}

Tank TH-1 is a 2500-gal stainless steel tank located in the Bethel Valley central laboratory area south of Building 3503. The underground tank was installed in 1948 and received waste from the thorium pilot plant project in Building 3503.

\subsubsection{Characterization Summary}

\subsubsection{Aqueous liquid}

The results for Tank TH-1 are shown in Tables 2.13.1 and 2.13.2. The samples from this tank were not significantly different from the blank. The TOC was among the lowest determined. None of the SVOA extracts was submitted for GC-MS because the GC screening did not reveal any constituents different from the blank. A precipitate was formed when sodium hydroxide was added to sample L50, and it was difficult to increase the $\mathrm{pH}$ above 10 even when concentrated sodium hydroxide solution was employed. 
Waste Characterization Data for ORNL Inactive LLLW Tanks

Rev. 0 / June 1992

Section 2.13 / page 2 of 3

Table 213.1. Analytical data for tank TH-1

\begin{tabular}{|c|c|c|c|c|c|}
\hline \multirow{2}{*}{ Parameter } & \multirow{2}{*}{$\begin{array}{c}\mathrm{DL} \\
(\mathrm{mg} / \mathrm{L})\end{array}$} & \multirow{2}{*}{ LA9 } & \multirow{2}{*}{ L50 } & \multicolumn{2}{|c|}{ RCRA limits } \\
\hline & & & & $(\mathrm{mg} / \mathrm{L})$ & $(\mathrm{mg} / \mathrm{kg})$ \\
\hline \multicolumn{6}{|c|}{ Physical properties and miscellaneous data } \\
\hline Density $(\mathrm{g} / \mathrm{mL})$ & & 1.034 & 1.034 & & \\
\hline Depth (inches) & & 4 & 10 & & \\
\hline Suspended solids (mg/mL) & 0.1 & $<0.1$ & $<0.1$ & & \\
\hline TDS $(\mathrm{mg} / \mathrm{mL})$ & 0.1 & 44.4 & 43.6 & & \\
\hline $\operatorname{TOC}(\mathrm{L}=\mathrm{mg} / \mathrm{L}, \mathrm{S}=\mathrm{mg} / \mathrm{kg})$ & 1.0 & 26.0 & 22.0 & & \\
\hline TS $(L=m g / m L, S=m g / g)$ & 0.1 & 41.3 & 35.5 & & \\
\hline \multicolumn{6}{|c|}{ RCRA mesals $(L=m g / L, S=m g / k g)$} \\
\hline Ag & 0.002 & 0.003 & 0.005 & 5 & 100 \\
\hline As & 0.40 & $<4$ & $<4$ & $s$ & 100 \\
\hline $\mathrm{Ba}$ & 0.02 & $<0.2$ & 0.23 & 100 & 2,000 \\
\hline $\mathrm{Cd}$ & 0.01 & $<0.2$ & $<0.2$ & 1 & 20 \\
\hline $\mathrm{Cr}$ & 0.10 & 1.2 & 1.4 & 5 & 100 \\
\hline $\mathrm{Hg}$ & 0.005 & 16 & 16 & 0.2 & 4.0 \\
\hline $\mathrm{Ni}$ & 0.10 & 1.5 & 1.7 & NA & NA \\
\hline $\mathrm{Pb}$ & 0.50 & $<2$ & 2.2 & 5 & 100 \\
\hline Se & 0.05 & $<0.09$ & $<0.09$ & 1 & 20 \\
\hline $\mathrm{T}$ & 0.05 & $<0.09$ & $<0.09$ & NA & NA \\
\hline \multicolumn{6}{|c|}{ Process metals ( $L=m g / L, S=m g / \mathrm{kg})$} \\
\hline $\mathrm{Si}$ & 1.0 & 35.3 & 38.3 & & \\
\hline $\mathrm{U}$ & 0.1 & 0.5 & 0.4 & & \\
\hline \multicolumn{6}{|c|}{ Anions $(L=m g / L, S=m g / k g)$} \\
\hline $\mathrm{Cl}^{-}$ & 1.0 & $<1$ & $<1$ & & \\
\hline $\mathrm{Cn}$ & 0.01 & $<0.04$ & $<0.10$ & & \\
\hline $\mathrm{F}$ & 1.0 & $<1$ & $<1$ & & \\
\hline $\mathrm{NO}_{3} \cdot$ & 5.0 & 1,400 & 1,300 & & \\
\hline $\mathrm{PO}_{4}^{-2}$ & 5.0 & $<250$ & $<250$ & & \\
\hline $\mathrm{SO}_{4}^{-2}$ & 5.0 & $<250$ & $<250$ & & \\
\hline \multicolumn{6}{|c|}{ Alkalinity } \\
\hline$H+(\mathrm{mol})$ & 0.01 & 0.41 & 0.40 & & \\
\hline $\mathrm{pH}$ (units) & 0.1 & 1.8 & 1.8 & & \\
\hline \multicolumn{6}{|c|}{ Radiochemical screening $(L=B q / \mathrm{mL}, S=B q / g)$} \\
\hline Gross alpha & & $3.9 \mathrm{E}+02$ & $4.2 \mathrm{E}+02$ & & \\
\hline Grass beta & & $3.2 \mathrm{E}+04$ & $3.9 \mathrm{E}+04$ & & \\
\hline \multicolumn{6}{|c|}{ Beta/gamma onitters $(L=B q / m L, S=B q / g)$} \\
\hline${ }^{137} \mathrm{Cs}$ & & $9.0 \mathrm{E}+03$ & $9.1 E+03$ & & \\
\hline${ }^{14} \mathrm{C}$ & & $5.0 \mathrm{E}-01$ & $5.0 \mathrm{E}-01$ & & \\
\hline${ }^{3} \mathrm{H}$ & & $5.0 \mathrm{E}+00$ & $4.9 \mathrm{E}+00$ & & \\
\hline${ }^{60} \mathrm{Co}$ & & $<20$ & $<20$ & & \\
\hline${ }^{90} \mathrm{Sr}$ & & $1.0 \mathrm{E}+04$ & $1.0 \mathrm{E}+04$ & & \\
\hline \multicolumn{6}{|c|}{ Alpha emitters $(L=B q / m L, S=B q(g)$} \\
\hline $233 \mathrm{U}$ & & 7.5E+01 & $8.8 \mathrm{E}+01$ & & \\
\hline${ }^{238} \mathrm{Pu}$ & & $<6$ & $<5$ & & \\
\hline${ }^{238} \mathrm{Th} / 232 \mathrm{Th}$ & & $4.0 \mathrm{E}+01$ & $5.1 \mathrm{E}+01$ & & \\
\hline${ }^{239} \mathrm{Pu}$ & & $<3$ & $<3$ & & \\
\hline${ }^{244} \mathrm{Cm}$ & & $<2$ & $<2$ & & \\
\hline${ }^{252} \mathrm{Cf}$ & & $<5$ & $<4$ & & \\
\hline
\end{tabular}

$D L=$ Detection limit 
Waste Characterization Data for ORNL Inactive LLLW Tanks

Rev. 0 / June 1992

Section 2.13 / page 3 of 3

Table 213.2. Organic anabytical data hits for tank TH-1

\begin{tabular}{|c|c|c|c|}
\hline Parameter & $\begin{array}{c}\mathrm{CRQL} \\
\mu \mathrm{g} / \mathrm{L}\end{array}$ & L49 & L50 \\
\hline \multicolumn{4}{|c|}{ Volatile organic componend anabuxis ( $L=\mu g / L, S=\mu g / \mathrm{kg}$ ) } \\
\hline Acetone & 10 & $18 \mathrm{~B}$ & $15 \mathrm{~B}$ \\
\hline Methylene chloride & 5 & $6 \mathrm{~B}$ & $6 \mathrm{~B}$ \\
\hline Toluene & 5 & $44 \mathrm{~B}$ & $45 \mathrm{~B}$ \\
\hline
\end{tabular}

$\mathrm{CRQL}=$ contract required quantitation limit 


\subsection{TANK TH-2}

\subsection{Tank Description}

Tank TH-2 is a 2400-gal stainless steel tank located in the Bethel Valley central laboratory area south of Building 3503. The underground tank was installed in 1952 and received waste from the thorium pilot plant project in Building 3503.

\subsubsection{Characterization Summary}

The results for this tank are shown in Tables 2.14.1 and 2.14.2.

\subsubsection{Aqueous liquid}

Two aqueous liquid samples, L131 and L132, were taken from tank TH-2. No sludge or organic layers were found. No TCL constituents were measured above the reporting limits $(5-10 \mu / \mathrm{L})$ except for toluene $(6 \mu \mathrm{g} / \mathrm{L}$ in both samples). The toluene was also found in the blank $(5 \mu \mathrm{g} / \mathrm{L})$; thus, it cannot be attributed to the tank contents. The main TICs were iodomethane and trichloroflouromethane, but they also were found in the blank; thus, they cannot be positively ascribed to the tank contents. Only in L132 were the TICs more concentrated (230 and $190 \mu \mathrm{g} / \mathrm{L}$ respectively) than in the blank ( 44 and $29 \mu \mathrm{g} / \mathrm{L}$ respectively). The very low results for the VOAs are consistent with the very low TOC content of $13.6 \mathrm{mg} / \mathrm{L}$ for $\mathrm{L} 131$ and $7.8 \mathrm{mg} / \mathrm{L}$ for $\mathrm{L} 132$.

Surrogate standard and matrix spike recoveries were generally good. One sample apparently had been double spiked with the surrogate standard. After correction for this mistake, the recoveries were very good. The matrix spike was omitted from the matrix spike duplicate sample, but the recoveries of the first matrix spike were very good. The blank was clean.

No compounds were detected in the major VOA compounds analysis. Matrix spike recoveries were very good, and the blank was clean.

No TCL constituents measured above the reporting limits $(250-1300 \mu \mathrm{g} / \mathrm{L})$ in either sample in the SVOA, although $52 \mu \mathrm{g} / \mathrm{L}$ of 2-chlorophenol was estimated in sample $\mathrm{L} 132$. Neither sample had TICs. The surrogate standard and matrix spike recoveries all were very good, and the blank was clean. 
Waste Characterization Data for ORNL Inactive LLLW Tanks

Rev. 0 / June 1992

Section 2.14 / page 2 of 3

Table 214.1. Analytical data for tank TH-2

\begin{tabular}{|c|c|c|c|c|c|}
\hline \multirow{2}{*}{ Parameter } & \multirow{2}{*}{$\begin{array}{c}D L \\
(\mathrm{mg} / \mathrm{L})\end{array}$} & \multirow{2}{*}{ L131 } & \multirow{2}{*}{$\mathrm{L} 132$} & \multicolumn{2}{|c|}{ RCRA limits } \\
\hline & & & & $(\mu \mathrm{g} / \mathrm{L})$ & $(\mu g / \mathbf{k g})$ \\
\hline \multicolumn{6}{|c|}{ Physical properties and miscellaneous data } \\
\hline Density $(\mathrm{g} / \mathrm{mL})$ & & 0.9989 & 1.0002 & & \\
\hline IC $(\mathrm{mg} / \mathrm{L})$ & 0.1 & $<0.1$ & 0.2 & & \\
\hline $\mathrm{TC}(\mathrm{mg} / \mathrm{L})$ & 1.0 & 13.6 & 8.0 & & \\
\hline TDS (mg/mL) & 0.1 & 4.9 & 5.0 & & \\
\hline $\operatorname{TOC}(L=m g / L, S=m g / k g)$ & 1.0 & 13.6 & 7.8 & & \\
\hline TS $(\mathrm{L}=\mathrm{mg} / \mathrm{mL}, \mathrm{S}=\mathrm{mg} / \mathrm{g})$ & 0.1 & 5.1 & 5.3 & & \\
\hline \multicolumn{6}{|c|}{$R C R A$ metals $(L=m g / h, S=m g / k g)$} \\
\hline Ag & 0.002 & $<0.2$ & $<0.2$ & 5 & 100 \\
\hline As & 0.40 & $<2$ & $<2$ & 5 & 100 \\
\hline $\mathrm{Ba}$ & 0.02 & $<0.1$ & $<0.1$ & 100 & 2000 \\
\hline $\mathrm{Cd}$ & 0.01 & 0.083 & $<0.08$ & 1 & 20 \\
\hline $\mathrm{Cr}$ & 0.10 & 0.80 & 0.68 & 5 & 100 \\
\hline $\mathrm{Hg}$ & 0.005 & 2.8 & 2.7 & 0.2 & 4 \\
\hline $\mathrm{Ni}$ & 0.10 & 0.58 & 0.43 & NA & NA \\
\hline $\mathrm{Pb}$ & 0.50 & 1.9 & 2.1 & 5 & 100 \\
\hline $\mathrm{Se}$ & 0.05 & $<1$ & $<1$ & 1 & 20 \\
\hline $\mathrm{T} 7$ & 0.05 & $<0.5$ & $<0.5$ & NA & NA \\
\hline \multicolumn{6}{|c|}{ Process metals $(L=m g / L, S=m g / k g)$} \\
\hline B & NA & $<0.7$ & $<0.7$ & & \\
\hline $\mathrm{Si}$ & 1.0 & $<1$ & $<1$ & & \\
\hline $\mathrm{Th}$ & NA & 1.68 & 1.71 & & \\
\hline $\mathrm{U}$ & 0.1 & $<8$ & $<8$ & & \\
\hline \multicolumn{6}{|c|}{ Anions $(L=m g / L, S=m g / k g)$} \\
\hline $\mathrm{Br}^{-}$ & 1.0 & 1510 & 1510 & & \\
\hline $\mathrm{Cl}^{+}$ & 1.0 & 18 & 18 & & \\
\hline $\mathrm{Cy}^{-}$ & 0.01 & 0.28 & 0.22 & & \\
\hline $\mathrm{F}$ & 1.0 & 17 & 17 & & \\
\hline $\mathrm{NO}_{3}^{-}$ & 5.0 & $<10$ & $<10$ & & \\
\hline $\mathrm{PO}_{4}^{-2}$ & 5.0 & $<10$ & $<10$ & & \\
\hline$S_{2}$ & 1.0 & $<0.1$ & $<0.1$ & & \\
\hline $\mathrm{SO}_{4}^{-2}$ & 5.0 & 26 & 26 & & \\
\hline \multicolumn{6}{|c|}{ Alkalinity } \\
\hline $\mathrm{H}+(\mathrm{mol})$ & 0.01 & 0.056 & 0.056 & & \\
\hline pH (units) & 0.1 & $244(212)$ & 2.46 & & \\
\hline \multicolumn{6}{|c|}{ Radiochemical screening ( $L=B q / m I, S=R q / g)$} \\
\hline Gross alpha & & $87.6(58)$ & 84.5 & & \\
\hline Gross beta & & $\begin{array}{c}9280 \\
(8690)\end{array}$ & $\begin{array}{l}9450 \\
(8730)\end{array}$ & & \\
\hline \multicolumn{6}{|c|}{ Betahgamma emitters $(L=B \mathrm{q} / \mathrm{mL}, \mathrm{S}=\mathrm{Bq} / \mathrm{g})$} \\
\hline${ }^{137} \mathrm{Cs}$ & & $\begin{array}{l}2710 \\
(2730)\end{array}$ & $\begin{array}{l}2740 \\
(2640)\end{array}$ & & \\
\hline${ }^{14} \mathrm{C}$ & & 2350 & $<1$ & & \\
\hline${ }^{3} \mathrm{H}$ & & 2.34 & 2.39 & & \\
\hline${ }^{60} \mathrm{Co}$ & & $4(3.6)$ & $<3(2.2)$ & & \\
\hline${ }^{90} \mathrm{Sr}$ & & $\begin{array}{l}2820 \\
(2780)\end{array}$ & $\begin{array}{l}2920 \\
(2720)\end{array}$ & & \\
\hline
\end{tabular}


Waste Characterization Data for ORNL Inactive LLLW Tanks Rev. 0 / June 1992

Table 214.1 (continued)

\begin{tabular}{|c|c|c|c|c|c|}
\hline \multirow{2}{*}{ Parameter } & \multirow{2}{*}{$\begin{array}{c}\mathrm{DL} \\
(\mathrm{mg} / \mathrm{L})\end{array}$} & \multirow{2}{*}{ L.131 } & \multirow{2}{*}{ L132 } & \multicolumn{2}{|c|}{ RCRA limits } \\
\hline & & & & $(\mu g / L)$ & $(\mu \mathrm{g} / \mathrm{kg})$ \\
\hline \multicolumn{6}{|c|}{ Alpha emitters ( $L=B q / m L, S=B q / g)$} \\
\hline${ }^{232} \mathrm{U}$ & & ND & 5 & & \\
\hline${ }^{233} U$ & & 15 & 17 & & \\
\hline${ }^{238} \mathrm{Pu}$ & & ND & ND & & \\
\hline${ }^{239} \mathrm{Pu} / 240 \mathrm{Pu}$ & & ND & ND & & \\
\hline${ }^{241} \mathrm{Am}$ & & ND & ND & & \\
\hline${ }^{244} \mathrm{Cm}$ & & ND & & & \\
\hline
\end{tabular}

$\mathrm{DL}=$ Detection limit

Table 214.2 Organic analytical data hits for tank TH-2

\begin{tabular}{|c|c|c|c|}
\hline Parameter & $\begin{array}{c}\text { CRQL } \\
(\mu g / L)\end{array}$ & $\begin{array}{r}\mathrm{L} 131 \\
(\mu \mathrm{g} / \mathrm{L}) \\
\end{array}$ & $\begin{array}{c}\mathrm{L} 132 \\
(\mu \mathrm{g} / \mathrm{L})\end{array}$ \\
\hline \multicolumn{4}{|c|}{ Volatile anganic composund anabusis } \\
\hline Toluene & 5 & 6 & 6 \\
\hline \multicolumn{4}{|c|}{ Semivolatile compound anabuis } \\
\hline 2-Chlorophenol & 10 & & 52 \\
\hline
\end{tabular}

$\mathrm{CRQL}=$ contract required quantitation limit 


\subsection{TANK TH-3}

\subsubsection{Tank Description}

Tank TH-3 is a 3300-gal stainless steel tank located in the Bethel Valley central laboratory area south of Building 3503. The underground tank was installed in 1952 and received waste from the thorium pilot plant project in Building 3503.

\subsubsection{Characterization Summary}

The results for this tank are shown in Tables 2.15.1 and 2.15.2.

\subsubsection{Aqueous liquid}

These samples were very similar to those drawn from tank TH-1. The lack of significant levels of organic compounds is reflected by the low TOC values, viz., $6.1,5.1$, and $0.8 \mathrm{mg} / \mathrm{L}$ for L52, L53, and L113, respectively. A precipitate formed during the basic $\mathrm{pH}$ adjustment of $\mathrm{L53}$, and achieving $\mathrm{pH}>10$ for the base/neutral extraction was difficult.

The absence of detectable compounds in L113, a blank submitted blind as a regular sample, is a good check of the entire handling, preparation, and analytical procedures for contamination. The toluene VOA matrix spike was not recovered, but the perdeuterated toluene surrogate standard was recovered well. 
Waste Characterization Data for ORNL Inactive LLLW Tanks

Rev. 0 / June 1992

Section 2.15 / page 2 of 3

Table 2.15.1. Anslytical data for tank TH-3

\begin{tabular}{|c|c|c|c|c|c|c|}
\hline \multirow{2}{*}{ Parameter } & \multirow{2}{*}{$\begin{array}{c}D L \\
(m g / L)\end{array}$} & \multirow{2}{*}{ LS2 } & \multirow{2}{*}{ LS3 } & \multirow{2}{*}{ L113 } & \multicolumn{2}{|c|}{ RCRA limits } \\
\hline & & & & & $(\mathrm{mg} / \mathrm{L})$ & $(\mathrm{mg} / \mathrm{kg})$ \\
\hline \multicolumn{7}{|c|}{ Physical properties and miscellaneous data } \\
\hline Density ( $(\mathrm{g} / \mathrm{mL})$ & & 1.015 & 1.015 & 1000 & & \\
\hline Depth (inches) & & 2.5 & 5 & NA & & \\
\hline Suspended solids $(\mathrm{mg} / \mathrm{mL})$ & 0.1 & $<0.1$ & $<0.1$ & 0.1 & & \\
\hline TDS $(\mathrm{mg} / \mathrm{mL})$ & 0.1 & 18.0 & 18.6 & $<0.1$ & & \\
\hline TOC $(\mathrm{mg} / \mathrm{L})$ & 1.0 & 6.1 & 5.1 & 0.8 & & \\
\hline $\mathrm{TS}(\mathrm{mg} / \mathrm{mL})$ & 0.1 & 15.1 & 15.6 & 0.1 & & \\
\hline \multicolumn{7}{|c|}{ RCRA metals $(L=m g / L, s=m g / k g)$} \\
\hline Ag & 0.002 & $<0.002$ & $<0.01$ & $<0.002$ & 5 & 100 \\
\hline As & 0.40 & $<2.1$ & $<2.1$ & $<02$ & 5 & 100 \\
\hline $\mathrm{Ba}$ & 0.02 & 0.89 & 0.86 & $<0.1$ & 100 & 2000 \\
\hline $\mathrm{Cd}$ & 0.01 & $<0.08$ & $<0.08$ & $<0.08$ & 1 & 20 \\
\hline $\mathrm{Cr}$ & 0.10 & 202 & 2.2 & $<0.1$ & 5 & 100 \\
\hline $\mathrm{Hg}$ & 0.005 & 11 & 9 & $<0.01$ & 0.2 & 4 \\
\hline $\mathrm{Ni}$ & 0.10 & 2.6 & 2.5 & $<0.2$ & NA & NA \\
\hline $\mathrm{Pb}$ & 0.50 & 1.9 & 2.2 & $<1.4$ & 5 & 100 \\
\hline Se & 0.05 & $<0.09$ & $<0.5$ & $<0.09$ & 1 & 20 \\
\hline $\mathrm{T} 1$ & 0.05 & $<0.09$ & $<0.5$ & $<0.09$ & NA & NA \\
\hline \multicolumn{7}{|c|}{ Process metals $(L=m g / L, S=m g / k g)$} \\
\hline $\mathbf{S i}$ & 1.0 & 15.7 & 18.2 & 3.23 & & \\
\hline $\mathrm{U}$ & 0.1 & $<0.1$ & $<0.1$ & $<0.1$ & & \\
\hline \multicolumn{7}{|c|}{ Anions ( $L=m g / L, S=m g / k g)$} \\
\hline $\mathrm{Cl}^{-}$ & 1.0 & $<1$ & $<1$ & $<1$ & & \\
\hline $\mathrm{Cn}$ & 0.01 & $<0.04$ & $<0.04$ & $<0.05$ & & \\
\hline $\mathrm{F}$ & 1.0 & $<1$ & $<1$ & $<1$ & & \\
\hline $\mathrm{NO}_{3} \cdot$ & 5.0 & 510 & 500 & $<5$ & & \\
\hline $\mathrm{PO}_{4}^{-2}$ & 5.0 & $<50$ & $<50$ & $<5$ & & \\
\hline $\mathrm{SO}_{4}^{-2}$ & 5.0 & $<50$ & $<50$ & $<5$ & & \\
\hline \multicolumn{7}{|c|}{ Alkalinity } \\
\hline $\mathrm{H}+(\mathrm{mol})$ & 0.01 & 0.17 & 0.18 & $\cdot$ & & \\
\hline $\mathrm{pH}$ (units) & 0.1 & 1.8 & 1.8 & 6.6 & & \\
\hline \multicolumn{7}{|c|}{ Radiochemical screening ( $L=B q / \mathrm{mL}, S=B q / g)$} \\
\hline Gross alpha & & $1.5 \mathrm{E}+02$ & $1.4 \mathrm{E}+02$ & $<1$ & & \\
\hline Gross beta & & $3.2 \mathrm{E}+04$ & $3.0 \mathrm{E}+04$ & $<20$ & & \\
\hline \multicolumn{7}{|c|}{ Beta/gamma emitters $(L=B q / m L, S=B q / g)$} \\
\hline${ }^{137} \mathrm{Cs}$ & & $7.7 \mathrm{E}+03$ & $8.4 \mathrm{E}+03$ & $<27$ & & \\
\hline${ }^{14} \mathrm{C}$ & & 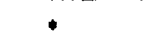 & $\cdot$ & $2.0 \mathrm{E}-01$ & & \\
\hline${ }^{3} \mathrm{H}$ & & $2.6 \mathrm{E}+00$ & $9.4 \mathrm{E}+00$ & $1.7 \mathrm{E}-01$ & & \\
\hline${ }^{60} \mathrm{Co}$ & & $<20$ & $<20$ & $<20$ & & \\
\hline${ }^{90} \mathrm{Sr}$ & & $8.8 \mathrm{E}+03$ & $8.7 \mathrm{E}+03$ & $7.2 \mathrm{E}-01$ & & \\
\hline \multicolumn{7}{|c|}{ Alphe emitiens ( $L=B q / m L, S=B q / g)$} \\
\hline${ }^{233} \mathrm{U}$ & & $2.6 \mathrm{E}+01$ & $7.1 \mathrm{E}+01$ & $<1$ & & \\
\hline${ }^{238} \mathrm{Pu}$ & & $<2$ & $<1$ & $<1$ & & \\
\hline${ }^{238} \mathrm{Th} / 232 \mathrm{Th}$ & & $1.7 \mathrm{E}+01$ & $1.5 \mathrm{E}+01$ & $<1$ & & \\
\hline${ }^{239} \mathrm{Pu}$ & & $<1$ & $<1$ & $<1$ & & \\
\hline${ }^{244} \mathrm{Cm}$ & & $<1$ & $<1$ & $<1$ & & \\
\hline${ }^{252} \mathrm{Cf}$ & & $<2$ & $<2$ & $\cdot$ & & \\
\hline
\end{tabular}

$\mathrm{DL}=$ Detection limit 
Waste Characterization Data for ORNL Inactive LLLW Tanks Rev. 0 / June 1992

Table 2.15.2. Organic anafytical data hits for tank TH1-3

\begin{tabular}{|c|c|c|c|c|c|}
\hline Parameter & $\begin{array}{l}\text { CRQL } \\
(\mu \mathrm{g} / \mathrm{L})\end{array}$ & LS2 & L53 & L113 & $\begin{array}{c}\text { RCRA } \\
\text { limit } \\
(\mu \mathrm{g} / \mathrm{L})\end{array}$ \\
\hline \multicolumn{6}{|c|}{ Volatile organic compound anabuis $(L=\mu g / L, S=\mu g / \mathrm{kg})$} \\
\hline Acetone & 10 & $16 \mathrm{~B}$ & $14 \mathrm{~B}$ & $45 \mathrm{~B}$ & \\
\hline Chlorobenzene & 5 & & & $2 \mathrm{~J}$ & 100,000 \\
\hline Methylene chloride & 5 & $8 \mathrm{~B}$ & $6 \mathrm{~B}$ & $4 \mathrm{JB}$ & \\
\hline Toluene & 5 & $7 \mathrm{~B}$ & $65 \mathrm{~B}$ & & \\
\hline \multicolumn{6}{|c|}{ Semivolatile compound analysis ( $L=m g / L, S=\mu g / \mu g)$} \\
\hline Bis(2-ethylhexyl)phthalate & 5 & & $79 \mathrm{~J}$ & & \\
\hline
\end{tabular}

$\overline{\mathrm{CRQL}}=$ contract required quantitation limit 


\subsection{TANK TH-4}

\subsubsection{Tank Description}

Tank TH-4 is a 14,000-gal Gunite tank located in the Bethel Valley central laboratory area south of Building 3503. The underground tank was installed in 1952 and received waste from the thorium and uranium pilot plant development projects in Building 3550.

\subsubsection{Characterization Summary}

Results for this tank are shown in Tables 2.16 .1 and 2.16.2.

\subsubsection{Aqueous liquid}

The aqueous samples from tank TH-4 were processed together. There was an insufficient amount of sample L117 for the SVOA extractions, so this sample received only the DAI-GC and VOA characterization.

The DAI-GC analysis indicated that the aqueous liquids contained methanol, acetone, 2-butanone, and 4-methyl-2-pentanone at high concentrations. VOA confirmed the presence of acetone, 4-methyl-2-butanone, and benzene; did not confirm the presence of 4-methyl2-pentanone; and did not detect methanol. The lower quantitative results for acetone and 4methyl-2-pentanone are probably because of the reduced purging efficiency of these species when present at milligram-per-liter levels.

Among the volatile TICs was di-isopropylether at $20 \mu \mathrm{g} / \mathrm{L}, 1000 \mu \mathrm{g} / \mathrm{L}, 1000 \mu \mathrm{g} / \mathrm{L}$, and $50 \mu \mathrm{g} / \mathrm{L}$ for $\mathrm{L} 55, \mathrm{~L} 56, \mathrm{~L} 57$, and $\mathrm{L} 117$, respectively.

Except for three two-ring and three-ring PAHs in L56, no semivolatile TCL organics were detected in the aqueous liquid samples. The major TIC was TBP, at 20 and $30 \mathrm{mg} / \mathrm{L}$ in L56 and L57, respectively.

\subsubsection{Sludges}

PAHs were found at milligram-per-kilogram concentrations in the sludges. TBP also was found as a TIC at $2 \mathrm{mg} / \mathrm{kg}$ in both sludges.

The sludges were hard to dry, even with an approximately fourfold-mass excess of sodium sulfate. However, the surrogate standard recoveries were good, except for nitrobenzene, which was usually recovered poorly, possibly because of evaporative loses during solvent concentration by nitrogen blowdown.

Except for the s!udges, the samples for tanks W-5, W-6, and W-7 were each processed as a group. 







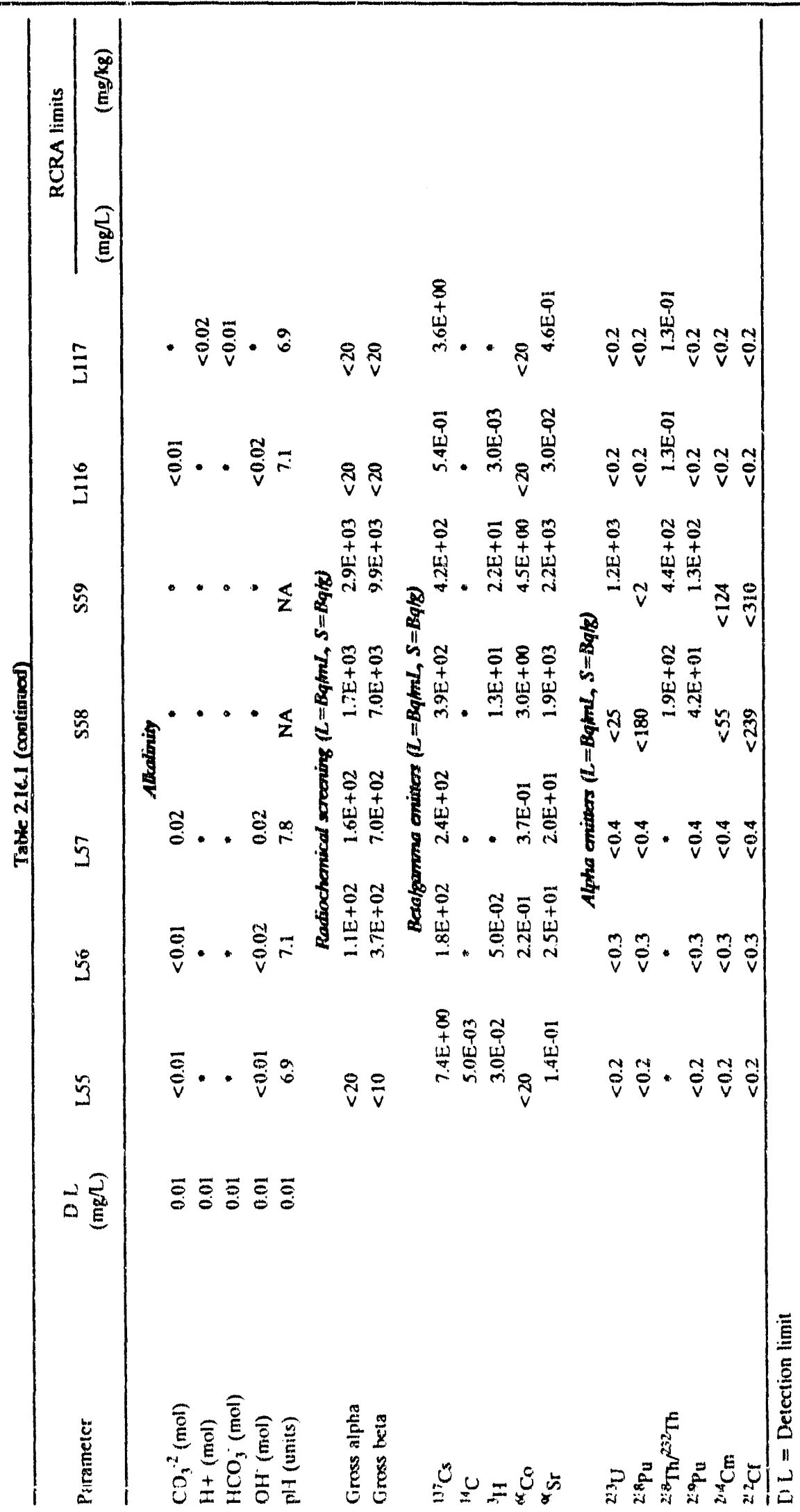




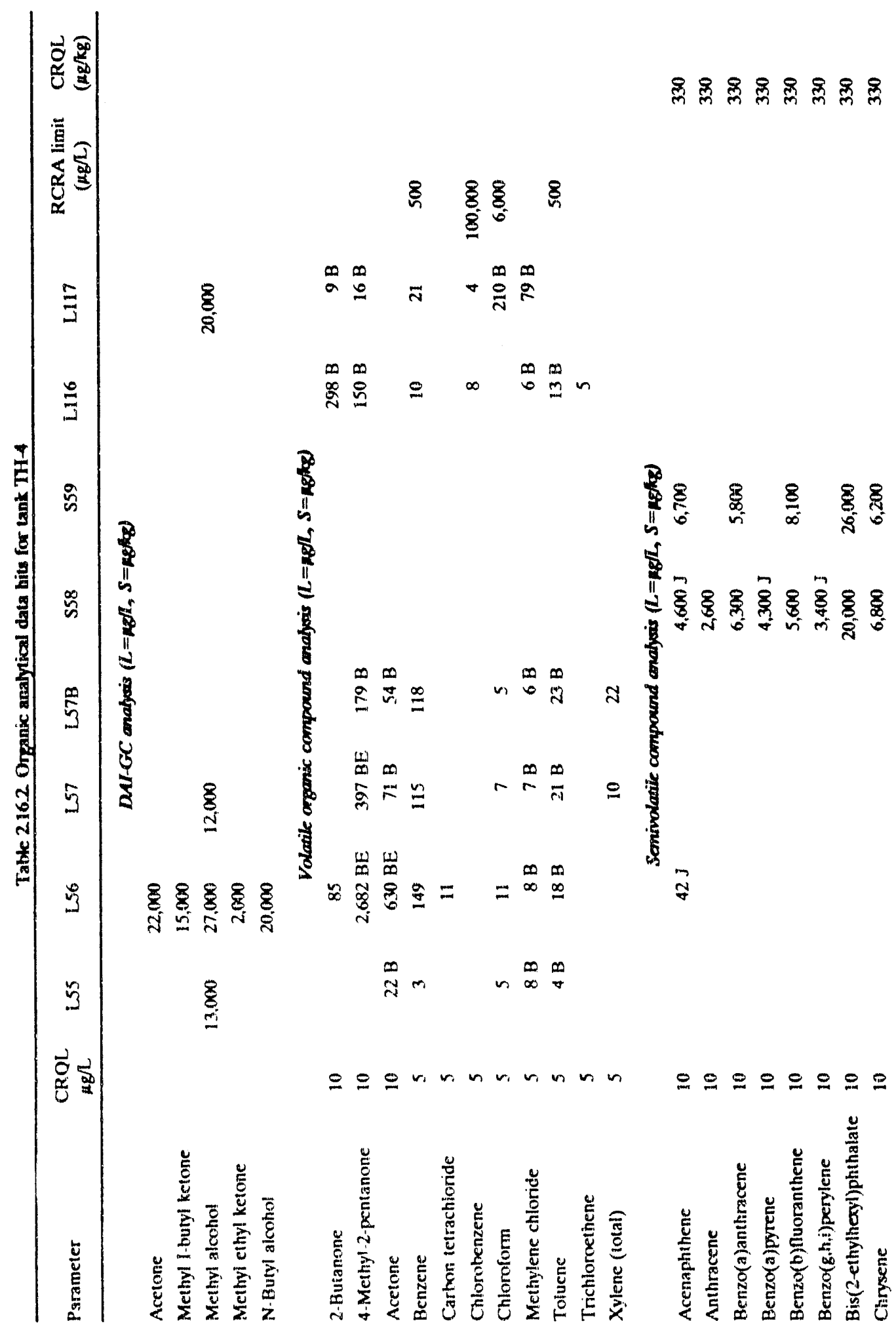




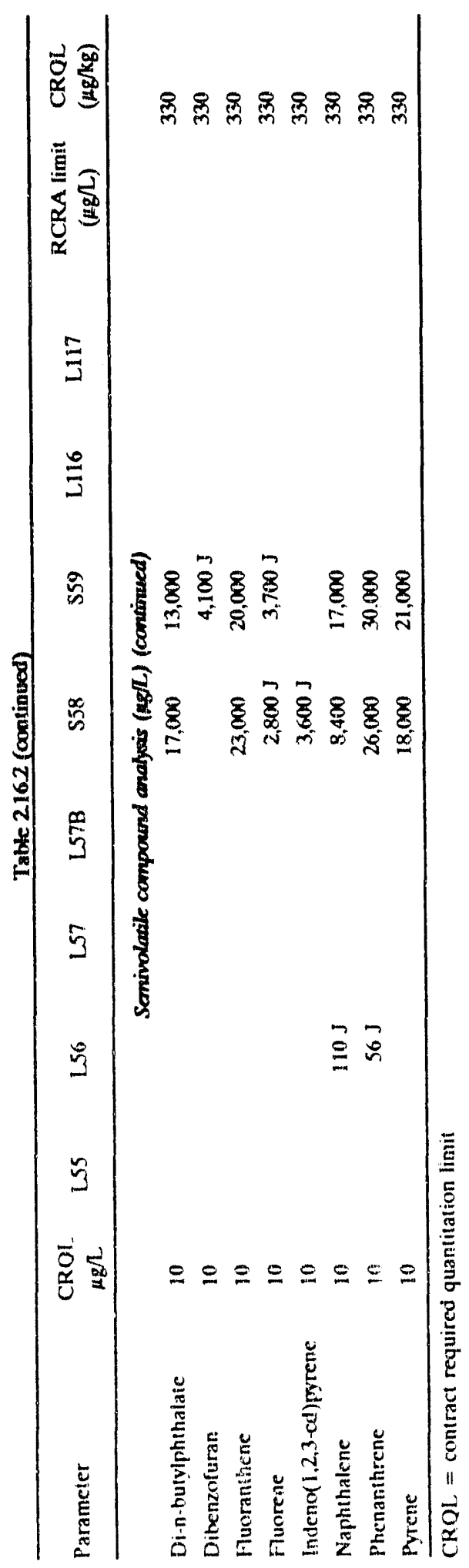




\section{TANK WC-1}

\subsubsection{Tank Description}

Tank WC-1 is a 2150-gal stainless steel tank located in the Isotopes Circle area of the Bethel Valley main laboratory area. The underground tank was installed in 1950 to collect LLLW from isotope production and development.

\subsubsection{Characterization Summary}

The results from this tank are shown in Tables 2.17.1 and 2.17.2.

\subsubsection{Aqueous liquid}

Two aqueous liquids samples, L124 and L134, were collected from this tank.

Two TCL constituents, methylene chloride and carbon disulfide, were detected in L134 at levels below the reporting limits. Methylene chloride was also detected in the blank at a comparable level. The carbon disulfide was very low (estimated at $3 \mu \mathrm{g} / \mathrm{L}$ ). Two unknown TICs were detected at a total of $44 \mu \mathrm{g} / \mathrm{L}$ in L134. These results are consistent with the low TOC found in these samples $(35 \mathrm{mg} / \mathrm{L}$ for $\mathrm{L} 124$ and $38 \mathrm{mg} / \mathrm{L}$ for $\mathrm{L} 134)$.

The recoveries of both the surrogate standards and the matrix spike were very low $(<14 \%)$ for these samples. The most likely cause is leakage in the new purge-and-trap apparatus used in the glove box. These samples will be rechecked after the apparatus is leakchecked and its performance is verified with the control samples.

No volatile organic compounds were detected in these samples. Matrix spikes were good, and the blanks were clean. These results support VOA findings.

No TCL constituents were found in either sample. Thirteen TICs totaled $13,000 \mu \mathrm{g} / \mathrm{L}$ in sample L134. Surrogate standard recoveries were good (but tribromophenol was above quality control iumits for water), and matrix spike recoveries were good except for phenol, 2-chlorophenol, 1,4-dichlorobenzene, and 1,2,4-trichlorobenzene in the matrix spike duplicate. The blank was clean. 
Waste Characterization Data for ORNL Inactive LLLW Tanks Rev. 0 / June 1992

Table 2.17.1. Analytical data for tank WC-1

\begin{tabular}{|c|c|c|c|c|c|c|}
\hline \multirow[b]{2}{*}{ Parameter } & \multirow{2}{*}{$\begin{array}{c}D \mathrm{~L} \\
(\mathrm{mg} / \mathrm{L})\end{array}$} & \multirow[b]{2}{*}{ L124 } & \multirow[b]{2}{*}{ L134 } & \multirow[b]{2}{*}{ L.134S } & \multicolumn{2}{|c|}{ RCRA limits } \\
\hline & & & & & $(\mathrm{mg} / \mathrm{L})$ & $(\mathrm{mg} / \mathrm{kg})$ \\
\hline \multicolumn{7}{|c|}{ Physical properties and miscellaneonus dasa } \\
\hline Density $(\mathrm{g} / \mathrm{mL})$ & & 0.9750 & 0.9760 & NA & & \\
\hline IC $(\mathrm{L}=\mathrm{mg} / \mathrm{L}, \mathrm{S}=\mathrm{mg} / \mathrm{kg})$ & 0.1 & 32 & 23 & 560 & & \\
\hline $\mathrm{TC}(\mathrm{L}=\mathrm{mg} / \mathrm{L}, \mathrm{S}=\mathrm{mg} / \mathrm{kg})$ & 1.0 & 67 & 61 & 9930 & & \\
\hline $\mathrm{TDS}(\mathrm{mg} / \mathrm{mL})$ & 0.1 & $<1$ & $<1$ & NA & & \\
\hline $\operatorname{TOC}(L=m g / L, S=m g / k g)$ & 1.0 & 35 & 38 & 9370 & & \\
\hline TS $(L=m g / m L, S=m g / g)$ & 0.1 & $<1$ & $<1$ & NA & & \\
\hline \multicolumn{7}{|c|}{$R C R A$ metals $(L=m g / L, S=m g / \mathrm{kg})$} \\
\hline$A_{8}$ & 0.002 & $<0.04$ & 0.33 & $<40$ & 5 & 100 \\
\hline As & 0.40 & 1.3 & 2.0 & $<300$ & 5 & 100 \\
\hline $\mathrm{Ba}$ & 0.02 & $<0.02$ & 93 & 927 & 100 & 2,000 \\
\hline $\mathrm{Cd}$ & 0.01 & $<0.02$ & 0.10 & 4.0 & 1 & 20 \\
\hline $\mathrm{Cr}$ & 0.10 & $<0.02$ & 2.9 & 112 & 5 & 100 \\
\hline $\mathrm{Hg}$ & 0.005 & $<2$ & $<2$ & 193 & 0.2 & 4 \\
\hline $\mathrm{Ni}$ & 0.10 & $<0.1$ & $<0.1$ & 10 & NA & NA \\
\hline $\mathrm{Pb}$ & 0.50 & $<0.2$ & 61 & 771 & 5 & 100 \\
\hline $\mathrm{Se}$ & 0.05 & 2.4 & $<0.02$ & $<20$ & 1 & 20 \\
\hline $\mathrm{T} 7$ & 0.05 & $<0.1$ & $<0.1$ & $<90$ & NA & NA \\
\hline \multicolumn{7}{|c|}{ Process metals $(L=m g / L, S=m g / k g)$} \\
\hline B & NA & $<0.04$ & $<0.04$ & $<40$ & & \\
\hline Si & 1.0 & 10 & 90 & NA & & \\
\hline $\mathrm{Th}$ & NA & $<0.3$ & $<0.3$ & $<200$ & & \\
\hline $\mathrm{U}$ & 0.1 & $<2$ & $<2$ & $<100$ & & \\
\hline \multicolumn{7}{|c|}{ Anions $(L=m g / L, S=m g / k g)$} \\
\hline $\mathrm{Br}^{-}$ & 1.0 & $<10$ & $<10$ & $<495$ & & \\
\hline $\mathrm{Cl}^{\circ}$ & 1.0 & 113 & 114 & 220 & & \\
\hline$C y^{\circ}$ & 0.01 & $<0.05$ & $<0.05$ & 0.33 & & \\
\hline $\mathrm{F}^{-}$ & 1.0 & 0.60 & $0.7 \mathrm{C}$ & 75 & & \\
\hline $\mathrm{NO}_{3}^{*}$ & 5.0 & $<10$ & $<10$ & 3,680 & & \\
\hline $\mathrm{PO}_{4}^{-2}$ & 5.0 & $<10$ & $<10$ & $<99$ & & \\
\hline $\mathrm{S}_{2}$ & 1.0 & $<0.1$ & 1.4 & 0.99 & & \\
\hline $\mathrm{SO}_{4}^{-2}$ & 5.0 & 196 & 204 & 449 & & \\
\hline \multicolumn{7}{|c|}{ Alkalininy } \\
\hline $\mathrm{pH}$ (units) & 0.1 & $8.25(6.50)$ & $7.46(6.39)$ & NA & & \\
\hline \multicolumn{7}{|c|}{ Radiochemical screening ( $L=B \mathrm{~g} / \mathrm{mL} L, S=B q \mathrm{~kg})$} \\
\hline Gross alpha & & $5(<50)$ & $286(1,560)$ & $\begin{array}{c}8.07 \mathrm{E}+04 \\
(8.62 \mathrm{E}+04)\end{array}$ & & \\
\hline Gross beta & & $\begin{array}{c}64,200 \\
(68,600)\end{array}$ & $\begin{array}{c}73,000 \\
(110,000)\end{array}$ & $\begin{array}{c}1.09 \mathrm{E}+06 \\
(1.36 \mathrm{E}+05)\end{array}$ & & \\
\hline
\end{tabular}


Waste Characterization Data for ORNL Inactive LLLW Tanks

Table 2.17 .1 (continued)

\begin{tabular}{|c|c|c|c|c|c|c|}
\hline \multirow[b]{2}{*}{ Parameter } & \multirow{2}{*}{$\begin{array}{c}D L \\
(\mathrm{mg} / \mathrm{L})\end{array}$} & \multirow[b]{2}{*}{$\mathrm{L} 124$} & \multirow[b]{2}{*}{ L134 } & \multirow[b]{2}{*}{ L134S } & \multicolumn{2}{|c|}{ RCRA limits } \\
\hline & & & & & $(\mathrm{mg} / \mathrm{L})$ & $(\mathrm{mg} / \mathrm{kg})$ \\
\hline \multicolumn{7}{|c|}{ Beta/grumma anitters $(L=B q / m I, S=B q / g)$} \\
\hline${ }^{137} \mathrm{Cs}$ & & $\begin{array}{c}59,200 \\
(58,000)\end{array}$ & $\begin{array}{c}61,700 \\
(56,500)\end{array}$ & $\begin{array}{c}2.69 E+05 \\
(2.73 E+05)\end{array}$ & & \\
\hline${ }^{14} \mathrm{C}$ & & 276 & 375 & $1.88 \mathrm{E}+04$ & & \\
\hline${ }^{152} \mathrm{Eu}$ & & $<30$ & $<30$ & $\begin{array}{c}<2.68 \mathrm{E}+02 \\
(<1.86 \mathrm{E}+03)\end{array}$ & & \\
\hline${ }^{154} \mathrm{Eu}$ & & $<30$ & $<30$ & $\begin{array}{c}4.12 \mathrm{E}+03 \\
(4.66 \mathrm{E}+03)\end{array}$ & & \\
\hline${ }^{155} \mathrm{Eu}$ & & $<150$ & $<150$ & $\begin{array}{c}3.16 E+03 \\
(4.12 E+03)\end{array}$ & & \\
\hline${ }^{241} \mathrm{Am}$ & & $<300$ & $\begin{array}{c}131 \\
(<300)\end{array}$ & $\begin{array}{c}1.57 E+04 \\
(1.73 E+04)\end{array}$ & & \\
\hline${ }^{3} \mathrm{H}$ & & 1,600 & 1,590 & $4.30 \mathrm{E}+02$ & & \\
\hline \multicolumn{7}{|c|}{ 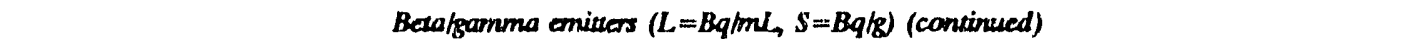 } \\
\hline${ }^{6} \mathrm{Co}$ & & $\begin{array}{c}767 \\
(852)\end{array}$ & $\begin{array}{c}432 \\
(594)\end{array}$ & $\begin{array}{l}1.30+05 \\
(1.29 \mathrm{E}+05)\end{array}$ & & \\
\hline${ }^{90} \mathrm{Sr}$ & & $\begin{array}{l}1,290 \\
(1,270)\end{array}$ & $\begin{array}{c}1,420 \\
(3,310)\end{array}$ & $\begin{array}{c}3.03 \mathrm{E}+05 \\
(4.03 \mathrm{E}+05)\end{array}$ & & \\
\hline \multicolumn{7}{|c|}{ Alpha emituess $(L=B q / m L, s=B q / g)$} \\
\hline${ }^{232} \mathrm{U}$ & & ND & ND & ND & & \\
\hline${ }^{233} \mathrm{U}$ & & ND & ND & ND & & \\
\hline${ }^{238} \mathrm{Pu}$ & & ND & 1110 & $4.06 \mathrm{E}+04$ & & \\
\hline${ }^{239} \mathrm{Pu} /{ }^{240} \mathrm{Pu}$ & & ND & ND & ND & & \\
\hline${ }^{241} \mathrm{Am}$ & & ND & 200 & $3.48 \mathrm{E}+04$ & & \\
\hline${ }^{244} \mathrm{Cm}$ & & ND & 251 & $1.08 \mathrm{E}+04$ & & \\
\hline
\end{tabular}

$\mathrm{D} L=$ Detection limit

Table 217.2. Organic anabytical data hits for tank WC-1

\begin{tabular}{|c|c|c|c|c|c|}
\hline Parameter & $\begin{array}{l}\text { CRQL } \\
(\mu g / L)\end{array}$ & L124 & L134 & $\begin{array}{c}\text { RCRA limit } \\
(\mu \mathrm{g} / \mathrm{L})\end{array}$ & $\begin{array}{l}\text { CRQL } \\
(\mu g / \mathrm{kg})\end{array}$ \\
\hline \multicolumn{6}{|c|}{ 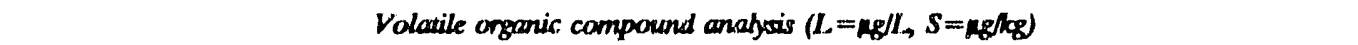 } \\
\hline Carbon disulfide & 5 & & 3 & 5 & \\
\hline Chloroform & 5 & & 1 & 5 & 6000 \\
\hline Methylene chloride & 5 & 240 & 160 & 5 & \\
\hline \multicolumn{6}{|c|}{ Semivolatile compournd anabusis $(L=\mathrm{g} / L, S=\mathrm{gg} / \mathrm{kg})$} \\
\hline Butylbenzylphthalate & 10 & & $190 \mathrm{~J}^{\mathrm{a}}$ & 10 & \\
\hline
\end{tabular}

-Inadvertently omitted from the original analysis report, ORNL./ER-19.

$\mathrm{CRQL}=$ contract requir " quantitation limit 


\subsection{TANK WC-15}

\subsubsection{Tank Description}

Tank WC-15 is a 1000-gal stainless steel tank located in the Building 4500 area of the Bethel Valley main laboratory area. The underground tank was installed in 1951 to collect LLLW from the Building 4500 research laboratories.

\subsubsection{Characterization Summary}

The results for this tank are shown in Tables 2.18.1 and 2.18.2.

\subsubsection{Aqueous liquid}

The samples from this tank consisted of three aqueous liquids (L126, L127, L128) and a thin oil layer (O130) that appeared much like 90-weight oil. These samples had very low levels of radioactivity. Because of the low radioactivity of these samples, the aqueous liquids were not prepared offline by remote purge-and-trap in a glove box, but rather they were prepared by direct purge-and-trap GC-MS. TCL constituents measured included traces of methylene chloride and 1,1,1-trichloroethane (also found in the blank) and traces of 1,2dichloroethene (14-15 $\mu \mathrm{g} / \mathrm{L}$ ). Carbon disulfide in $\mathrm{L} 128$ (estimated at $1 \mu \mathrm{g} / \mathrm{L}$ ) was essentially insignificant. All three samples contained the TIC ethyl ether $(-15-17 \mu \mathrm{g} / \mathrm{L})$, and L128 also exhibited two unknown TICs totalling $9 \mu \mathrm{g} / \mathrm{L}$. These very low concentrations of volatile organic compounds are consistent with the low TOC determinations of $21 \mathrm{mg} / \mathrm{L}, 19 \mathrm{mg} / \mathrm{L}$, and $24 \mathrm{mg} / \mathrm{L}$ for L126, L127, and L128, respectively. Surrogate standard recoveries were all within quality control limits, but the holding time for groundwater samples exceeded the time allowed by $7 \mathrm{~d}$.

The oil sample did not contain TCL compounds or TICs detectable by the VOA; this indicates that it is a relatively heavy oil devoid of volatile organic matter. It apparently does not have an appreciable solubility in the aqueous layer.

No major volatile organic compounds were detected in the aqueous samples. Major VOA is not appropriate for the oil layer, and that sample was not analyzed. Matrix spike recoveries were good, and the blank was clean.

SVOA did not reveal any TCL constituents or TICs for the aqueous samples. The oil layer did have one TCL, di-n-butylphthalate, at $1900 \mu \mathrm{g} / \mathrm{L}$. The essentially hydrocarbon nature of the oil is suggested by the 15 unk.nown hydrocarbon TICs totalling $-189,000 \mu \mathrm{g} / \mathrm{kg}$, the 3 unknown TICs totally $-39,000 \mu \mathrm{g} / \mathrm{kg}$, and the 2 TICs apparently consisting of brominated benzene derivatives (possibly bromodiethyl benzenes), all of which added up to $14,000 \mu \mathrm{g} / \mathrm{kg}$. The sum of the TCL constituents and the TICs is $\sim 243,800 \mu \mathrm{g} / \mathrm{kg}(\sim 0.02 \mathrm{wt} \%)$. The bulk of the GC-MS profile was a large unresolved hump (typical of heavy oil).

The surrogate standard and matrix spike recoveries were all good except for the slightly low 2-fluorobiphenyl in L-127 and the lack of recovery of 4-nitrophenol in both the matrix spike and matrix spike duplicate. The blank was clean. 
Negative ion, chemical ionization GC-MS was used to examine the oil sample (O-130) for PCBs and polybrominated biphenyls. Neither were detected. However, chlorinated aromatic compounds were detected, and these would explain the response observed in the $\mathrm{PCB}$ analysis. Identification of these chlorinated compounds is continuing.

Table 2 18.1. Analytical data for tank WC-15

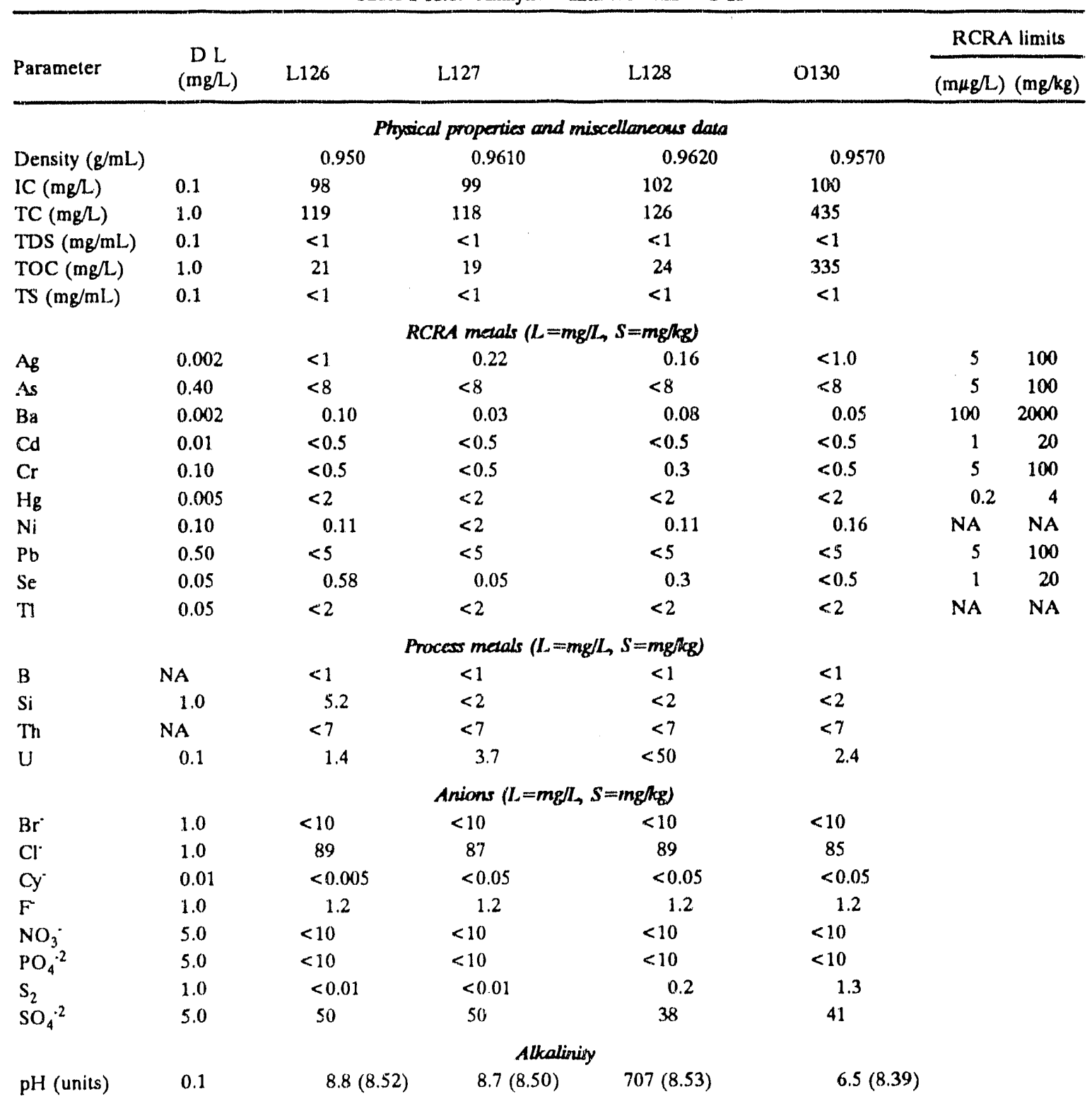


Waste Characterization Data for ORNL Inactive LLLW Tanks Rev. 0/June 1992

Table 2.18 .1 (continued)

\begin{tabular}{|c|c|c|c|c|c|c|}
\hline \multirow[b]{2}{*}{ Parameter } & \multirow{2}{*}{$\begin{array}{c}\mathrm{DL} \\
(\mathrm{mg} / \mathrm{L})\end{array}$} & \multirow[b]{2}{*}{ L126 } & \multirow[b]{2}{*}{ L127 } & \multirow[b]{2}{*}{ L128 } & \multirow[b]{2}{*}{0130} & \multirow{2}{*}{$\frac{\text { RCRA limits }}{(\mathrm{m} \mu \mathrm{g} / \mathrm{L})(\mathrm{mg} / \mathrm{kg})}$} \\
\hline & & & & & & \\
\hline \multicolumn{7}{|c|}{ Radiochanical screening ( $L=B q / m L, S=B q / g)$} \\
\hline Gross alpha & & $0.89(<1)$ & $0.13(<1)$ & $0.43(<1)$ & $0.56(<1)$ & \\
\hline Gross beta & & $11.7(<10)$ & $10.3(<10)$ & $14.6(<10)$ & $15.4(<10)$ & \\
\hline \multicolumn{7}{|c|}{ Betalgamma amitters $\left(L=B q / m L_{,} S=B q g_{g}\right)$} \\
\hline${ }^{137} \mathrm{Cs}$ & & $3.0(<5)$ & $3.1(<5)$ & $4.0(<5)$ & $3.5(<5)$ & \\
\hline${ }^{14} \mathrm{C}$ & & $<1$ & $<1$ & 33 & 95 & \\
\hline${ }^{3} \mathrm{H}$ & & 18.4 & 18.6 & 18.9 & 18.5 & \\
\hline${ }^{60} \mathrm{Co}$ & & $<0.2$ & $<0.2$ & $<0.2$ & $<0.3$ & \\
\hline${ }^{90} \mathrm{Sr}$ & & $1.40(<10)$ & $1.71(<10)$ & $1.93(<10)$ & $3.36(<10)$ & \\
\hline
\end{tabular}

$\mathrm{DL}=$ Detection limit

Table 2.18.2 Organic analytical data hits for tant WC-15

\begin{tabular}{|c|c|c|c|c|c|c|c|}
\hline Parameter & $\begin{array}{c}\text { CRQL } \\
\mu \mathrm{g} / \mathrm{L}\end{array}$ & $L 126$ & L127 & L128 & 0130 & $\begin{array}{c}\text { RCRA } \\
\operatorname{limit} \\
(\mu \mathrm{g} / \mathrm{L})\end{array}$ & $\begin{array}{l}\text { CRQL } \\
(\mu \mathrm{g} / \mathrm{kg})\end{array}$ \\
\hline
\end{tabular}

Volatile organic compound analysis $(L=\mu g / L, S=\mu g / \mathrm{kg})$

1,1,1-Tricholoroethane

$\begin{array}{cccc}5 & 16 & & \\ 5 & 15^{a} & 15 & 14 \\ 5 & & & 1 \\ 5 & 18 & & \\ 5 & & & \end{array}$

Semivolatile compound anahusis ( $L=\mu g / L, S=\mu g / k g$ )

1,2-Dichloroethene (total)

Carbon disulfide

Chloroform

.

${ }^{a}$ Did not appear in the RAP data base.

$\mathrm{CRQL}=$ contract required quantitation limit 


\subsection{TANK WC-17}

\subsection{Tank Description}

Tank WC-17 is a 1000-gal stainless steel tank located in the Building 4500 area of the Bethel Valley main laboratory area. The underground tank was installed in 1951 to collect LLLW from the Building 4500 research laboratories.

\subsubsection{Characterization Summary}

The results for this tank are shown in Tables 2.19.1 and 2.19.2.

\subsubsection{Aqueous liquid}

This complex sample set, prepared as a group, consisted of three samples having an organic layer floating on an aqueous liquid, as well as two additional aqueous liquids. The activity of these samples was low enough that both the aqueous and organic layers could be subjected to purge-and-trap GC-MS in the GC-MS laboratory. Small volumes were diluted in water at the time of purge and trap because of the expected high concentrations of volatile organic compounds. There was insufficient sludge S106 for SVOA.

The main compounds identified in the VOA were acetone, benzene, methylene chloride, and xylene. However, considering the high blank levels, only xylene appears to be present in the sample at 50-80 $\mu \mathrm{g} / \mathrm{L}$. The reason for the high blank level is not known. The lack of detectable organic compounds in the DAI-GC supports the contention that most of the VOA results were blank-related.

In the SVOA, only phthalates were detected at levels similar to those in the blank.

Considering the elevated levels of compounds detected in the VOA blank, only styrene (at 5000 to $8000 \mu \mathrm{g} / \mathrm{L}$ ) can be confidently identified in the organic layer. Trichloroethylene and vinyl acetate also were found, but in only one of the samples. SVOA detected only phthalates, which appear to be related to the blank. TICs were mostly unknown hydrocarbons, except for 1,1-biphenyl-2-o1 (200 mg/kg in all three organic layers). A low level of PCBs was determined in the organic layers. This appeared to be a mixture of Aroclors 1254 and 1260, with a total concentration of $29-33 \mathrm{mg} / \mathrm{kg}$.

The samples from tanks W.13, W-14, W-15, and W-1A were each processed together. 


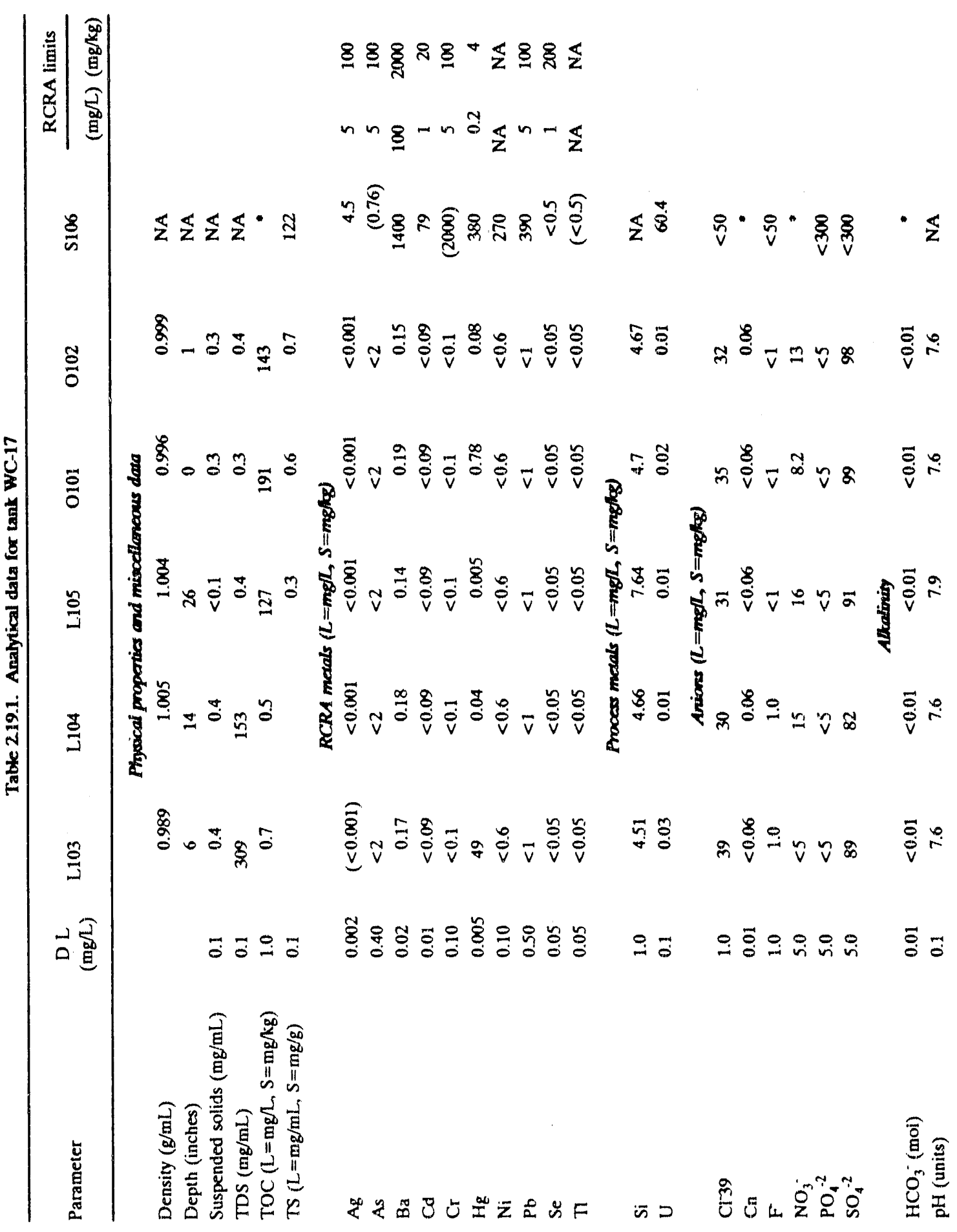




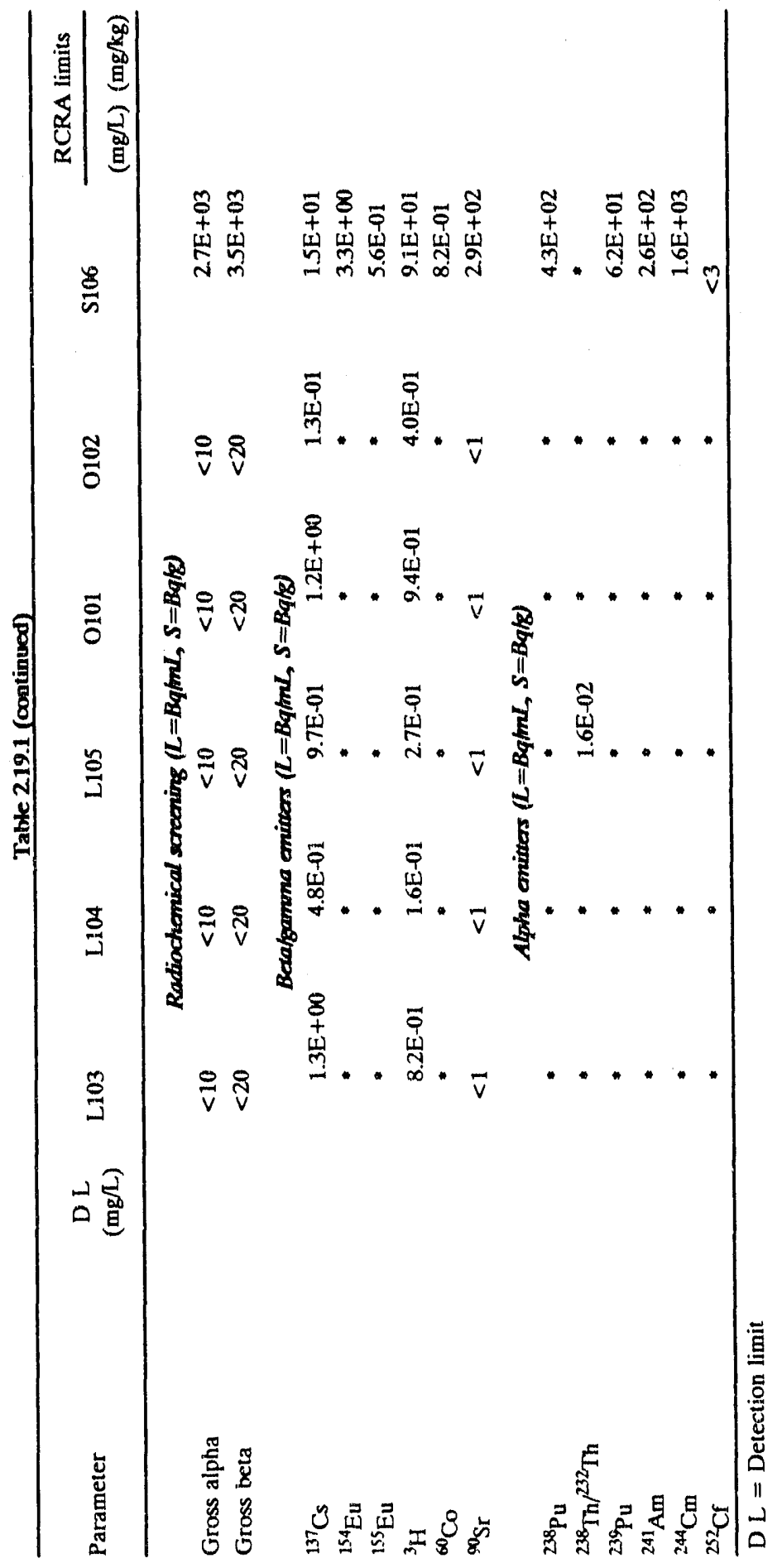




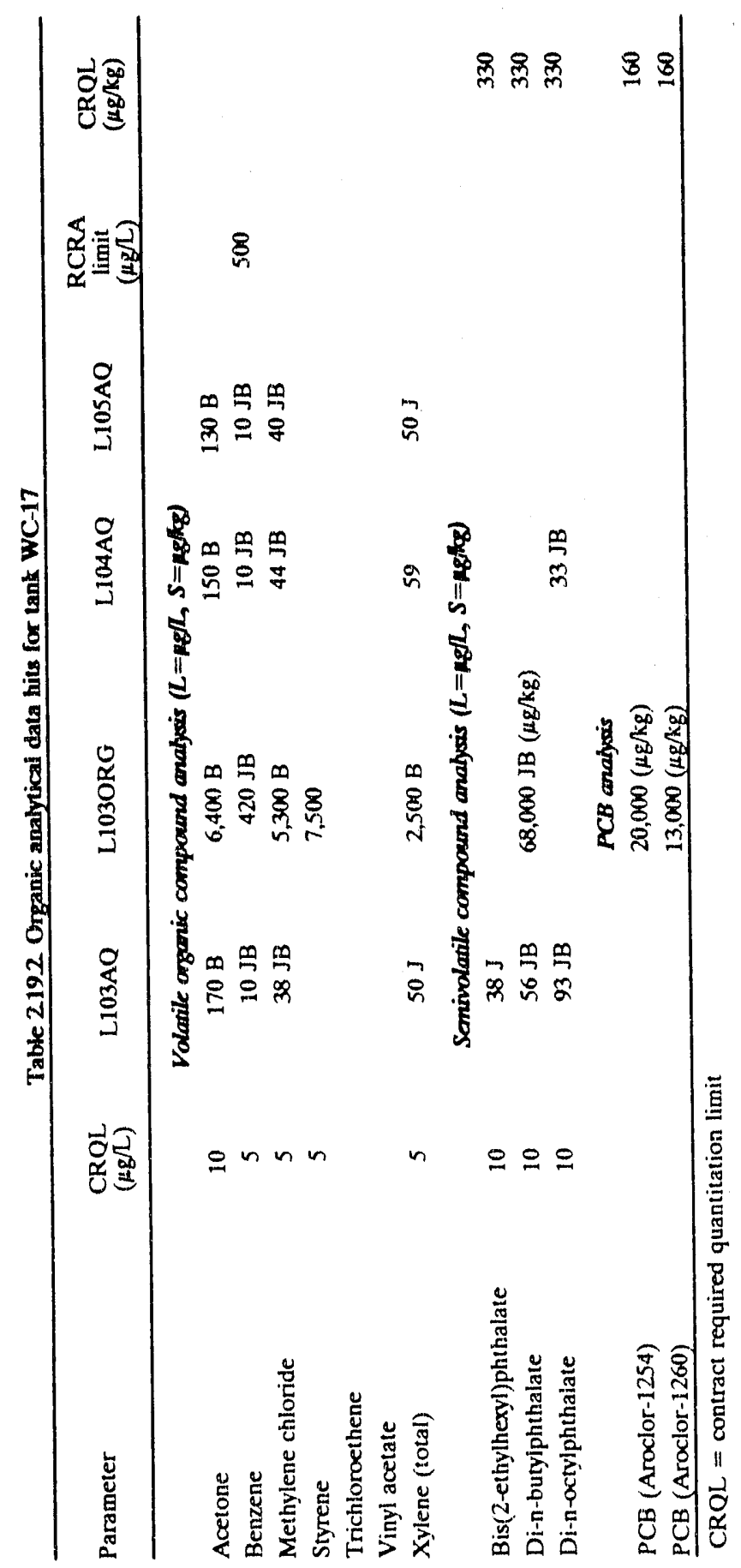




\subsection{TANK W-1}

\subsubsection{Tank Description}

Tank W-1 is a 4800-gal Gunite tank located in the Bethel Valley North Tank Farm. The underground tank was constructed in 1943 and received LLLW from the Building 3019 Radiochemical Processing Facility.

\subsubsection{Characterization Summary}

The results from this tank are shown in Tables 2.20.1 and 2.20.2.

\subsubsection{Aqueous liquid}

No significant concentrations of TCL compounds were detected in the samples from this tank. This is consistent with the very low TOC, 6.4 and $10.1 \mathrm{mg} / \mathrm{L}$ for L7 and L8, respectively. Two aliquots from $\mathrm{L} 7$ underwent VOA. The VOA surrogate standard recoveries were very poor for the A aliquot (10-19\%) but were good for the B aliquot. The latter should be considered the more accurate analysis. 
Waste Characterization Data for ORNL Inactive LLLW Tanks

Rev. 0 / June 1992

Table 220.1. Analyzical data for tank W-1

\begin{tabular}{|c|c|c|c|c|}
\hline Parameter & $\begin{array}{c}D L \\
(m g / L)\end{array}$ & 1.7 & L8 & $\begin{array}{r}\text { RCRA } \\
\text { limits } \\
(\mathrm{mg} /)\end{array}$ \\
\hline \multicolumn{5}{|c|}{ Phystical properties and miscellaneous data } \\
\hline Density $(\mathrm{g} / \mathrm{mL})$ & & 1.002 & 1.002 & \\
\hline Depth (inches) & & 4 & 9 & \\
\hline Suspended solids (mg/mL) & 0.1 & $<0.1$ & $<01$ & \\
\hline $\mathrm{TDS}(\mathrm{mg} / \mathrm{mL})$ & 0.1 & 0.2 & 0.2 & \\
\hline $\operatorname{TOC}(\mathrm{mg} / \mathrm{L})$ & -1.0 & 6.4 & 10.1 & \\
\hline $\mathrm{TS}(\mathrm{mg} / \mathrm{mL})$ & 0.1 & 0.2 & 0.2 & \\
\hline \multicolumn{5}{|c|}{$R C R A$ metals $(L=m g / L, S=m g / k g)$} \\
\hline $\mathrm{Ag}$ & 0.0102 & $<0.002$ & $<0.002$ & 5.0 \\
\hline As & 2.0 & $<2$ & $<2$ & 5.0 \\
\hline $\mathrm{Ba}$ & 0.2 & 0.13 & 0.11 & 100.0 \\
\hline $\mathrm{Cd}$ & 0.04 & $<0.04$ & $<0.04$ & 1.0 \\
\hline $\mathrm{Cr}$ & 0.1 & $<0.1$ & $<0.1$ & 5.0 \\
\hline $\mathrm{Hg}$ & 0.01 & $<0.01$ & $<0.01$ & 0.2 \\
\hline $\mathrm{Ni}$ & 0.6 & $<0.6$ & $<0.6$ & NA \\
\hline $\mathrm{Pb}$ & 1.0 & $<1$ & $<1$ & 5.0 \\
\hline $\mathrm{Se}$ & 0.19 & $<0.09$ & $<0.09$ & 1.0 \\
\hline $\mathrm{T}$ & 0.09 & $(<0.09)$ & $(<0.09)$ & NA \\
\hline \multicolumn{5}{|c|}{ Phoi mesals $(L=m g / L, s=m g / k g)$} \\
\hline $\mathrm{Si}$ & & 8.38 & 8.69 & NA \\
\hline$U$ & 0.1 & $<0.1$ & $<0.1$ & NA \\
\hline \multicolumn{5}{|c|}{ Anions $\left(L=m g / L_{\rightarrow} S=m p / g g\right)$} \\
\hline $\mathrm{Cl}^{\circ}$ & 1.0 & 13 & 13 & \\
\hline $\mathrm{Cn}$ & -0.01 & $<0.06$ & $<0.05$ & \\
\hline $\mathrm{F}$ & 1.0 & 6.1 & 6.2 & \\
\hline $\mathrm{NO}_{3}$ & 5.0 & 32 & 32 & \\
\hline $\mathrm{PO}_{4}^{-2}$ & 5.0 & $<5$ & $<5$ & \\
\hline $\mathrm{SO}_{4}^{-2}$ & 5.0 & 47 & 47 & \\
\hline \multicolumn{5}{|c|}{ Alkaliniry } \\
\hline $\mathrm{CO}_{3}^{2}(\mathrm{~mol})$ & 0.01 & $<0.01$ & $<0.61$ & \\
\hline $\mathrm{HCO}_{3}(\mathrm{~mol})$ & 0.01 & $<0,01$ & $<0.01$ & \\
\hline pH (units) & 0.1 & 7.9 & 8.0 & \\
\hline \multicolumn{5}{|c|}{ Radicathemical screaring ( $L=B q / \mathrm{ml}, S=B q$ g) } \\
\hline Gross alpha & & $<3$ & $<3$ & \\
\hline Grosss betás & & B.7E +02 & $8.7 E+02$ & \\
\hline \multicolumn{5}{|c|}{ 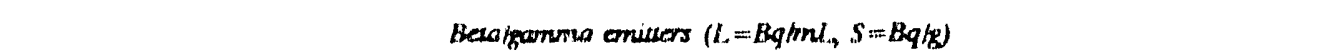 } \\
\hline${ }^{127} \mathrm{Cs}$ & & $1.8 \mathrm{E}+01$ & $1.8 \mathrm{E}+01$ & \\
\hline 3 & & $3.9 \mathrm{E}+\infty$ & $2.5 E+(0)$ & \\
\hline${ }^{4} \mathrm{Co}$ & & $<10$ & $<10$ & \\
\hline
\end{tabular}


Waste Characterization Data for ORNL Inactive LLLW Tanks? Rev. 0 / June 1992

Table 20.2 Organic analytical dats hits for tank W-1

\begin{tabular}{|c|c|c|c|c|}
\hline Parameter & $\begin{array}{c}\text { CRQL } \\
(\mu \mathrm{g} / \mathrm{L})\end{array}$ & L7 & L7B & 1.8 \\
\hline \multicolumn{5}{|c|}{ Volatile onganic compound anabusis $(L=n g / L, S=\mu g / \mathrm{kg})$} \\
\hline Methylene chlorive & 5 & & $10 \mathrm{~B}$ & $11 \mathrm{~B}$ \\
\hline Toluene & 5 & & $5 \mathrm{~B}$ & $11 \mathrm{~B}$ \\
\hline \multicolumn{5}{|c|}{ Semivolutile compound anabsis $(L=\mu g / L, S=\mu g / \alpha g)$} \\
\hline Di-n-butylphthalate & 10 & $9 \mathrm{~J}$ & & $8 \mathrm{~J}$ \\
\hline
\end{tabular}

$\mathrm{CRQL}=$ contract required quantitation limit 


\subsection{TANK W-1A}

\subsection{Tank Description}

Tank W-1A is a 4000-gal stainless steel tank located in the Bethel Valley North Tank Farm. The underground tank was installed in 1951 and received waste from the Radiochemical Processing Facility (Building 3019) and the HRLAL (Buildings 3026 and 3019-B).

\subsection{Characterization Summary}

The results for this 'ank are shown in Tables 2.21.1 and 2.21.2.

\subsubsection{Aqueous liquid}

The results for this tank were very similar to the results for WC-15, WC-17, and W-1. DAI-GC determined $7 \mathrm{mg} / \mathrm{L}$ of methanol, and only very low levels of volatiles were detected by VOA. SVOA determined only a phthalate. TICs included $\mathrm{C}_{4}$-benzenes (probably diethylbenzenes used in radiochemical extractions) at $5 \mathrm{mg} / \mathrm{L}$ and ethylphenylethanone at $3 \mathrm{mg} / \mathrm{L}$.

The samples for tanks T-30 and 7562 were each processed together. 
Waste Characterization Data for ORNL Inactive LLLW Tanks Rev. 0/June 1992

Table 2.21.1. Analynical data for tank W-1A

\begin{tabular}{lcccc}
\hline & $\mathrm{DL}$ & \multicolumn{2}{c}{ RCRA limits } \\
Parameter & $\mathrm{mg} / \mathrm{L})$ & $\mathrm{L}$ & $(\mathrm{mg} / \mathrm{L})$ & $(\mathrm{mg} / \mathrm{kg})$ \\
\hline
\end{tabular}

Density $(g / \mathrm{mL})$

Depth (inches)

Suspended solids ( $\mathrm{mg} / \mathrm{mL}$ )

TDS (mg/mL)

IS $(\mathrm{L}=\mathrm{mg} / \mathrm{mL}, \mathrm{S}=\mathrm{mg} / \mathrm{g})$

\section{Ag}

As

$\mathrm{Ba}$

$\mathrm{Cd}$

$\mathrm{Cr}$

$\mathrm{Hg}$

$\mathrm{Ni}$

$\mathrm{Pb}$

Se

T)

Si

U

$\mathrm{Cl}^{-}$

$\mathrm{Cn}$

$\mathrm{F}$

$\mathrm{NO}_{3}{ }^{-}$

$\mathrm{PO}_{4}^{-2}$

$\mathrm{SO}_{4}^{-2}$

$\mathrm{HCO}_{3}(\mathrm{~mol})$

pH (units)

Gross alpha

Gross beta

${ }^{134} \mathrm{Cs}$
${ }^{137} \mathrm{Cs}$
${ }^{3} \mathrm{H}$
${ }_{610} \mathrm{Co}$
${ }^{404} \mathrm{Sr}$

${ }^{232} \mathrm{U}$

${ }^{233} \mathrm{U}$

${ }^{238} \mathrm{Pu}$

${ }^{23.8} \mathrm{U}$

${ }^{239} \mathrm{Pu}$

${ }^{241} \mathrm{Am}$

${ }^{244} \mathrm{Cm}$

$x^{3} \mathrm{Ci}$

$\overline{\mathrm{DL}}=$ Detection limil
Physical properies and miscellaneous data

$\begin{array}{lccr} & 1.002 & & \\ 0.1 & 2 & & \\ 0.1 & 0.3 & & \\ 0.1 & 0.4 & & \\ & 0.7 & & \\ 0.002 & \text { RCRA metals }(L=m g / L, S=m g / k) & & 100 \\ 0.40 & 0.008 & 5 & 100 \\ 0.02 & <4 & 5 & 2000 \\ 0.01 & <0.2 & 100 & 20 \\ 0.10 & 7.3 & 1 & 100 \\ 0.005 & 0.33 & 5 & 4 \\ 0.10 & 0.96 & 0.2 & \text { NA } \\ 0.50 & <1 & \text { NA } & 20 \\ 0.05 & <2 & 5 & \text { NA } \\ 0.05 & <0.09 & 1 & \text { NA } \\ & <0.09 & & \end{array}$

Phocess metals ( $L=m g / L, S=m g / \mathrm{g}_{\mathrm{g}}$ ) 4.47

477

Anions ( $L=m g / L, S=m g / k g)$

33

$<0.06$

1.0

$<5$

$<5$

58.0

Alkalinisy

$<0.01$

0.01

0.1

8.8

Radiachernical screening (Bq/mLl.)

$1.1 \mathrm{E}+03$

$1.3 E+03$

Besalgarnma emisters (Bq/mL)

$4.6 \mathrm{E}+00$

$1.4 \mathrm{~F}:+02$

2. $6 \mathrm{E}+(0)$

$4.4 \mathrm{E}+00$

$3.2 \mathrm{E}+02$

Alpha aniteas (Bghtorl.)

$2.7 \mathrm{E}+02$

$7.3 \mathrm{E}+02$

$1.9 \mathrm{E}+01$

$5.0 \mathrm{E}+\infty 0$

$6.7 \mathrm{E}+01$

$5.4 E+01$

$9.0 \mathrm{E}+00$ $=2$ 
Waste Characterization Data for ORNL Inactive LLLW Tanks

Rev. 0 / June 1992

Section 2.21 / page 3 of 3

Table 221.2 Onganic anałylical data hits for tant W-1A

\begin{tabular}{|c|c|c|c|}
\hline Parameter & $\begin{array}{l}\text { CRQL } \\
(\mu \mathrm{g} / \mathrm{L})\end{array}$ & Ll & $\begin{array}{c}\text { RCRA limit } \\
(\mu \mathrm{g} / \mathrm{L})\end{array}$ \\
\hline \multicolumn{4}{|c|}{ DAI-GC anabois $(L,=M g / L, S=A g / k g)$} \\
\hline Methyl alcohol & & 7000 & \\
\hline \multicolumn{4}{|c|}{ Volatile organic compound anabusis $(L=\mu g / L, S=\mu g / \mathrm{kg})$} \\
\hline Acetone & 10 & $17 \mathrm{~B}$ & \\
\hline Benzene & 5 & $3 \mathrm{JB}$ & 500 \\
\hline Chlorobenzene & 5 & $3 \mathrm{~J}$ & 100,000 \\
\hline Ethylbenzene & 5 & $4 \mathrm{~J}$ & \\
\hline Methylene chloride & 5 & $4 \mathrm{JB}$ & \\
\hline Toluene & 5 & $63 \mathrm{~B}$ & \\
\hline Trichloroethene & 5 & $20 \mathrm{~B}$ & 500 \\
\hline Xylene (total) & 5 & 13 & \\
\hline \multicolumn{4}{|c|}{ Semivolatile campound analyois (ng/L) } \\
\hline Diethylphthalate & 10 & $24 \mathrm{~J}$ & \\
\hline
\end{tabular}

$\mathrm{CRQL}=$ contract required quantitation limit 


\subsection{TANK W-2}

\section{2, 1 Tanfikescription}

Tank W-2 is a 4800 -gal Gunite tank located in the Bethel Valley North Tank Farm. The underground tank was constructed in 1943 and received LLLW from the Building 3019 Radiochemical Processing Facility.

\subsection{2 .2 Characterization Summary}

The results for this tank are shown in "Tables 2.22.1 and 2.22.2.

\subsubsection{Aqueous liquid}

Neither sample contained significant levels of TCL constituents. This is consistent with the low TOCs of $18.9 \mathrm{mg} / \mathrm{L}$ for $\mathrm{L} 11$ and $22.6 \mathrm{mg} / \mathrm{L}$ for $\mathrm{L} 118$. These samples were collected in series and are useful as field duplicates, within normal sampling errors.

The acidic surrogate standard compounds were very poorly recovered from the aqueous liquids, suggesting that acidic compounds, if present in the samples, may not have been extracted or detected. The two chlorinated phenolic matrix spike compounds were also poorly recovered. 
Waste Characterization Data for ORNL Inactive LLLW Tanks Rev. 0 / June 1992

Table 222.1. Analytical data for tank W-2

\begin{tabular}{|c|c|c|c|c|c|}
\hline \multirow{2}{*}{ Parameter } & \multirow{2}{*}{$\begin{array}{c}\mathrm{D} \mathrm{L} \\
(\mathrm{mg} / \mathrm{L})\end{array}$} & \multirow{2}{*}{ L11 } & \multirow{2}{*}{ L118 } & \multicolumn{2}{|c|}{ RCRA limits } \\
\hline & & & & $(\mathrm{mg} / \mathrm{L})$ & $(\mathrm{mg} / \mathrm{kg})$ \\
\hline \multicolumn{6}{|c|}{ Physical properties and miscellaneous data } \\
\hline Density $(g / m L)$ & & 1.002 & 1.003 & & \\
\hline Depth (inches) & & 4 & 4 & & \\
\hline Suspended solids (mg/mL) & 0.1 & $<0.1$ & 0.2 & & \\
\hline TDS $(\mathrm{mg} / \mathrm{mL})$ & 0.1 & 0.4 & 0.3 & & \\
\hline $\mathrm{TOC}(\mathrm{mg} / \mathrm{L})$ & 1.0 & 18.9 & 22.6 & & \\
\hline TS $(\mathrm{mg} / \mathrm{mL})$ & 0.1 & 0.3 & 0.5 & & \\
\hline \multicolumn{6}{|c|}{$R C R A$ metals (mg/L) } \\
\hline Ag & 0.002 & $<0.002$ & $<0.002$ & 5 & 100 \\
\hline As & 0.40 & $<2$ & $<2$ & 5 & 100 \\
\hline $\mathrm{Ba}$ & 0.02 & 0.19 & 0.14 & 100 & 2000 \\
\hline $\mathrm{Cd}$ & 0.01 & $<0.04$ & $<0.04$ & 1 & 20 \\
\hline $\mathrm{Cr}$ & 0.10 & $<0.1$ & $<0.1$ & 5 & 100 \\
\hline $\mathrm{Hg}$ & 0.005 & $<0.01$ & $<0.01$ & 0.2 & 4 \\
\hline $\mathrm{Ni}$ & 0.10 & $<0.5$ & $<0.6$ & NA & NA \\
\hline $\mathrm{Pb}$ & 0.50 & 1.1 & $<1$ & 5 & 100 \\
\hline $\mathrm{Se}$ & 0.05 & $<0.09$ & $<0.09$ & 1 & 20 \\
\hline $\mathrm{T} 1$ & 0.05 & $(<0.09)$ & $(<0.09)$ & NA & NA \\
\hline \multicolumn{6}{|c|}{ Process metals (mg/L) } \\
\hline $\mathrm{Si}$ & 1.0 & 4.94 & 4.5 & & \\
\hline $\mathrm{U}$ & 0.1 & 0.18 & 0.17 & & \\
\hline \multicolumn{6}{|c|}{ Anions (mg/L) } \\
\hline $\mathrm{Cl}^{-}$ & 1.0 & 7.2 & 7.6 & & \\
\hline $\mathrm{Cn}$ & 0.01 & $<0.05$ & $<0.05$ & & \\
\hline $\mathrm{F}$ & 1.0 & 9.2 & 9.9 & & \\
\hline $\mathrm{NO}_{3}$ & 5.0 & $<5$ & $<5$ & & \\
\hline $\mathrm{PO}_{4}^{-2}$ & 5.0 & $<5$ & $<5$ & & \\
\hline $\mathrm{SO}_{4}^{-2}$ & 5.0 & 15 & 15 & & \\
\hline \multicolumn{6}{|c|}{ Alkalinity } \\
\hline $\mathrm{CO}_{3}{ }^{2}(\mathrm{~mol})$ & 0.01 & $<0.01$ & $<0.01$ & & \\
\hline $\mathrm{HC})_{3}(\mathrm{~mol})$ & 0.01 & $<0.01$ & $<0.01$ & & \\
\hline $\mathrm{pH}$ (units) & 0.1 & 8.5 & 8.5 & & \\
\hline \multicolumn{6}{|c|}{ Radiachemical screening (Bq/mL) } \\
\hline Gross alpha & & $<3$ & $<3$ & & \\
\hline Gross beta & & $7.1 \mathrm{E}+02$ & $7.4 \mathrm{E}+02$ & & \\
\hline \multicolumn{6}{|c|}{ Besalgamma conitters (Bq/mL) } \\
\hline${ }^{137} \mathrm{Cs}$ & & $2.2 E+01$ & $2.0 \mathrm{E}+01$ & & \\
\hline${ }^{3} \mathrm{H}$ & & $1.5 \mathrm{E}+00$ & $1.7 \mathrm{E}+00$ & & \\
\hline${ }^{\infty} \mathrm{Co}$ & & $<10$ & $<10$ & & \\
\hline
\end{tabular}

DL $=$ Detection limit 
Waste Characterization Data for ORNL Inactive LLLW Tanks

Rev. 0 / June 1992

Section 2.22/ page 3 of 3

Table 2222. Organic analytical data hits for tank W-2

\begin{tabular}{|c|c|c|c|}
\hline Parameter & $\begin{array}{l}\text { CRQL } \\
(\mu \mathrm{g} / \mathrm{L}) \\
\end{array}$ & L11 & L118 \\
\hline \multicolumn{4}{|c|}{ Volatile organic compound analysis (mg/L) } \\
\hline Methylene chloride & 5 & $10 \mathrm{~B}$ & $9 \mathrm{~B}$ \\
\hline Toluene & 5 & & $15 \mathrm{~B}$ \\
\hline \multicolumn{4}{|c|}{ Semivolatile compound anajusis (ng/L) } \\
\hline Di-n-butylphthalate & 10 & $10 \mathrm{~J}$ & \\
\hline
\end{tabular}

$\mathrm{CRQL}=$ contract required qual itation limit 


\subsection{TANK W-3}

\subsubsection{Tank Description}

Tank W-3 is a 42,500-gal Gunite tank located in the Bethel Valley North Tank Farm. The underground tank was constructed in 1943 and received LLLW from the Building 3019 Radiochemical Processing Facility.

\subsubsection{Characterization Summary}

The results for this tank are shown in Tables 2.23.1 and 2.23.2.

\subsubsection{Aqueous liquid}

The analytical results for these samples, which were prepared as a group, are listed in Table 2.23. The major organic compound was methanol. DAI-GC also determined $\mathrm{mg} / \mathrm{L}$ levels of acetone and 2-butanone that were not confirmed by VOA. Very low concentrations of other volatiles were detected by VOA. SVOA determined very low levels of phthalates that were not significantly different from those in the blank. PAHs were also detected in L18, which had a TOC approximately double that of the other two liquids. TBP was estimated at $30 \mathrm{mg} / \mathrm{L}$ as a TIC in L16, L17, and L18.

\subsubsection{Sludge}

As in other samples, somewhat higher levels of PAHs and phthalates were measured in the sludge. The latter were not significantly different in concentration from the blank. TBP was estimated at $4 \mathrm{mg} / \mathrm{kg}$ as a TIC. 
Waste Characterization Data for ORNL Inactive LLLW Tanks

Rev. 0 / June 1992

Section 2.23 / page 2 of 3

Table 2.23.1. Anarytical data for tank W-3

\begin{tabular}{|c|c|c|c|c|c|c|c|}
\hline \multirow{2}{*}{ Parameter } & \multirow{2}{*}{$\underset{(\mathrm{mg} / \mathrm{L})}{\mathrm{DL}}$} & \multirow{2}{*}{ L16 } & \multirow{2}{*}{$\mathrm{L} 17$} & \multirow{2}{*}{ L.18 } & \multirow{2}{*}{ S19 } & \multicolumn{2}{|c|}{ RCRA limits } \\
\hline & & & & & & $(\mathrm{mg} / \mathrm{L})$ & $(\mathrm{mg} / \mathrm{kg})$ \\
\hline \multicolumn{8}{|c|}{ Physical properties and miscellaneous data } \\
\hline Density $(\mathrm{g} / \mathrm{mL})$ & & 1.004 & 1.006 & 1.009 & NA & & \\
\hline Depth (inches) & & 4 & 60 & 106 & NA & & \\
\hline Suspended solids $(\mathrm{mg} / \mathrm{mL})$ & 0.1 & $<0.1$ & $<0.1$ & $<0.1$ & NA & & \\
\hline TDS $(\mathrm{mg} / \mathrm{mL})$ & 0.1 & 2.9 & 4.6 & 8.6 & NA & & \\
\hline $\operatorname{TOC}(\mathrm{L}=\mathrm{mg} / \mathrm{L}, \mathrm{S}=\mathrm{mg} / \mathrm{kg})$ & 1.0 & 772 & 1130 & 1500 & 3410 & & \\
\hline$T S(L=m g / m L, S=m g / g)$ & 0.1 & 2.7 & 4.4 & 8.4 & 300 & & \\
\hline \multicolumn{8}{|c|}{$R C R A$ metals $(L=m g / L, S=m g / k g)$} \\
\hline Ag & 0.002 & 0.002 & 0.002 & 0.002 & 0.05 & 5 & 100 \\
\hline As & 0.40 & $<4$ & $<4$ & $<4$ & $(<2)$ & 5 & 100 \\
\hline $\mathrm{Ba}$ & 0.02 & $<0.2$ & $<0.2$ & $<0.2$ & $(6.4)$ & 100 & 2000 \\
\hline Cd & 0.01 & $<0.2$ & $<0.2$ & $<0.2$ & 0.52 & 1 & 20 \\
\hline $\mathrm{Cr}$ & 0.10 & 3.3 & 6.8 & 20 & 1300 & 5 & 100 \\
\hline $\mathrm{Hg}$ & 0.005 & $<0.01$ & 0.01 & 0.02 & 0.83 & 0.2 & 4 \\
\hline $\mathrm{Ni}$ & 0.10 & $<1$ & $<1$ & $<1$ & $<7$ & NA & NA \\
\hline $\mathrm{Pb}$ & 0.50 & $<2$ & $<2$ & $<2$ & 52 & 5 & 100 \\
\hline $\mathrm{Se}$ & 0.05 & $<0.09$ & 0.09 & $<0.09$ & $(<0.3)$ & 1 & 20 \\
\hline $\mathrm{T}$ & 0.05 & $<0.09$ & $<0.09$ & $<0.09$ & $<0.3$ & NA & NA \\
\hline \multicolumn{8}{|c|}{ Process metals ( $L=m g / L, S=m g / \mathrm{kg})$} \\
\hline $\mathrm{Si}$ & 1.0 & 6.5 & 5.3 & 7.2 & NA & & \\
\hline $\mathrm{U}$ & 0.1 & 88 & 163 & 285 & 61300 & & \\
\hline \multicolumn{8}{|c|}{ Anions $(L=m g / L, S=m g / k g)$} \\
\hline $\mathrm{Cl}^{\circ}$ & 1.0 & 7.8 & 7.9 & 10.0 & $<100$ & & \\
\hline $\mathrm{Cn}$ & 0.01 & $<0.08$ & $<0.07$ & 0.10 & * & & \\
\hline $\mathrm{F}$ & 1.0 & $<10$ & $<10$ & $<10$ & $<100$ & & \\
\hline $\mathrm{NO}_{3}^{-}$ & 5.0 & $<50$ & $<50$ & $<50$ & * & & \\
\hline $\mathrm{PO}_{4}^{-2}$ & 5.0 & 490 & 600 & 800 & $<500$ & & \\
\hline $\mathrm{SO}_{4}^{-2}$ & 5.0 & 280 & 430 & 620 & $<500$ & & \\
\hline \multicolumn{8}{|c|}{ Alkalinity } \\
\hline $\mathrm{CO}_{3}^{-2}(\mathrm{~mol})$ & 0.01 & $<0.01$ & 0.02 & 0.05 & * & & \\
\hline $\mathrm{HCO}_{3}^{\circ}\left(\mathrm{mol}^{\prime}\right.$ & 0.01 & 0.03 & $\cdot$ & • & - & & \\
\hline $\mathrm{pH}$ (units) & 0.1 & 9.0 & 10.1 & 11.1 & NA & & \\
\hline \multicolumn{8}{|c|}{ Radiochemical screening ( $L=B q / m I, S=B q / g)$} \\
\hline Gross alpha & & $6.0 \mathrm{E}+00$ & $1.2 \mathrm{E}+01$ & $1.7 \mathrm{E}+01$ & $8.4 \mathrm{E}+03$ & & \\
\hline Gross beta & & $4.9 \mathrm{E}+02$ & $6.7 E+02$ & $1.2 \mathrm{E}+03$ & $1.5 \mathrm{E}+06$ & & \\
\hline \multicolumn{8}{|c|}{ Betalgamme emitsers $(L=B q / m i, S=B q / g)$} \\
\hline${ }^{137} \mathrm{Cs}$ & & $5.8 \mathrm{E}+02$ & $8.4 \mathrm{E}+02$ & $1.3 \mathrm{E}+0.3$ & $4.7 \mathrm{E}+04$ & & \\
\hline${ }^{154} \mathrm{Eu}$ & & $\cdot$ & $\cdot$ & $\cdot$ & $7.6 \mathrm{E}+01$ & & \\
\hline${ }^{3} \mathrm{H}$ & & $6.9 \mathrm{E}-01$ & $9.4 \mathrm{E}-01$ & $1.1 \mathrm{E}+00$ & $2.2 \mathrm{E}+00$ & & \\
\hline${ }^{60} \mathrm{Co}$ & & $<10$ & $1.0 \mathrm{E}-01$ & $2.6 \mathrm{E}-01$ & $2.4 \mathrm{E}=01$ & & \\
\hline${ }^{90} \mathrm{Sr}$ & & $4.0 \mathrm{E}+01$ & $6.4 \mathrm{E}=01$ & $9.0 \mathrm{E}+01$ & $1.5 \mathrm{E}+0.5$ & & \\
\hline
\end{tabular}


Waste Characterization Data for ORNL Inactive LLLW Tanks Rev. 0 / June 1992

Table 2.23 .1 (continued)

\begin{tabular}{|c|c|c|c|c|c|c|c|}
\hline \multirow{2}{*}{ Parameter } & \multirow{2}{*}{$\underset{(\mathrm{mg} / \mathrm{L})}{\mathrm{DL}}$} & \multirow{2}{*}{ L16 } & \multirow{2}{*}{ L17 } & \multirow{2}{*}{ L18 } & \multirow{2}{*}{ S19 } & \multicolumn{2}{|c|}{ RCRA limits } \\
\hline & & & & & & $(\mathrm{mg} / \mathrm{L})$ & $(\mathrm{mg} / \mathrm{kg})$ \\
\hline \multicolumn{8}{|c|}{ Alphe emitters $(L=B q / m L, S=B q / g)$} \\
\hline${ }^{233} \mathrm{U}$ & & $<5$ & $<5$ & $<5$ & $1.1 \mathrm{E}+03$ & & \\
\hline${ }^{238} \mathrm{Pu}$ & & $<5$ & $<5$ & $<5$ & $1.7 \mathrm{E}+02$ & & \\
\hline${ }^{238} \mathrm{U}$ & & $*$ & $\bullet$ & $\cdot$ & $4.3 \mathrm{E}+02$ & & \\
\hline${ }^{239} \mathrm{Pu}$ & & $<5$ & $<5$ & $<5$ & $3.9 E+03$ & & \\
\hline${ }^{241} \mathrm{Am}$ & & $\bullet$ & $\cdot$ & - & $2.6 \mathrm{E}+02$ & & \\
\hline${ }^{244} \mathrm{Cm}$ & & $<5$ & $<5$ & $<5$ & $<10$ & & \\
\hline${ }^{252} \mathrm{Cf}$ & & $<5$ & $<5$ & $<5$ & $<10$ & & \\
\hline
\end{tabular}

$\mathrm{DL}=$ Detection limit

Table 2.23.2. Organic analytical data hits for tank W-3

\begin{tabular}{|c|c|c|c|c|c|c|}
\hline Parameter & $\begin{array}{l}\text { CRQL } \\
(\mu \mathrm{g} / \mathrm{L})\end{array}$ & L16 & L17 & L18 & $\$ 20$ & $\begin{array}{c}\text { RCRA } \\
\text { limit } \\
(\mu g / L)\end{array}$ \\
\hline \multicolumn{7}{|c|}{$D A I-G C$ analysir $(L=\mu g / L, S=\mu g / \mathrm{kg})$} \\
\hline Acetone & & & & 7,000 & & \\
\hline Methyl alcohol & & 38,000 & 40,000 & 20,000 & & \\
\hline Methyl ethyl ketone & & & & 5,000 & & \\
\hline N-Butyl alcohol & & & & 7,000 & & \\
\hline \multicolumn{7}{|c|}{ Volatile ongronic compound anabyxis $(L=\mu g / L, S=\mu g / \mathrm{gg})$} \\
\hline 4-Methyl-2-pentanone & 10 & & & 18 & & \\
\hline Acetone & 10 & $2 \mathrm{JB}$ & $26 \mathrm{~B}$ & $5 \mathrm{JB}$ & & \\
\hline Benzene & 5 & & & $4 \mathrm{~J}$ & & \\
\hline Chlorobenzene & 5 & & & 8 & & 100,000 \\
\hline Chloroform & 5 & & $1 \mathrm{~J}$ & & & 6,000 \\
\hline Methylene chloride & 5 & $4 \mathrm{JB}$ & $4 \mathrm{JB}$ & $3 \mathrm{JB}$ & & \\
\hline Tetrachloroethene & 5 & $6 \mathrm{~B}$ & $8 \mathrm{~B}$ & & & 700 \\
\hline Toluene & 5 & $5 \mathrm{JB}$ & $14 \mathrm{~B}$ & $37 \mathrm{~B}$ & & \\
\hline Trichloroethene & 5 & $11 \mathrm{~B}$ & $14 \mathrm{~B}$ & $11 \mathrm{~B}$ & & 500 \\
\hline \multicolumn{7}{|c|}{ Semivolatile compound anabusis $(L=m g / L, S=M g / k g)$} \\
\hline 2-Nitrophenol & 10 & & & $53 \mathrm{~J}$ & & \\
\hline Bis(2-ethylhexyl)phthalate & 10 & & & & $23 \mathrm{JB}$ & 330 \\
\hline Chrysene & 10 & & & & $4 \mathrm{~J}$ & 330 \\
\hline Di-n-butylphthalate & 10 & $60 \mathrm{~J}$ & $48 \mathrm{~J}$ & $230 \mathrm{~J}$ & $47 \mathrm{JB}$ & 330 \\
\hline Di-n-octylphthalate & 10 & $49 \mathrm{~J}$ & $80 \mathrm{~J}$ & & & \\
\hline Fluoranthene & 10 & & & $56 \mathrm{~J}$ & & \\
\hline Phenanthrene & 10 & & & $33 \mathrm{~J}$ & $12 \mathrm{~J}$ & 330 \\
\hline Pyrene & 10 & & & $60 \mathrm{~J}$ & & \\
\hline
\end{tabular}

$\mathrm{CRQL}=$ contract required quantitation limit 


\subsection{TANK W-4}

\subsubsection{Tank Description}

Tank W-4 is a 42,500-gal Gunite tank located in the Bethel Valley North Tank Farm. The underground tank was constructed in 1943 and received LLLW from the Building 3019 Radiochemical Processing Facility.

\subsubsection{Characterization Summary}

The results from this tank are shown in Tables 2.24.1 and 2.24.2.

\subsubsection{Aqueous liquid}

Some of the samples from this tank contained elevated levels of organic compounds. Sample $\mathrm{L} 23$ contained 4-methyl-2-pentanone ( $7 \mathrm{mg} / \mathrm{L}$ by DAI-GC, $1.1 \mathrm{mg} / \mathrm{L}$ by VOA). The VOA was not effective for determining $\mathrm{mg} / \mathrm{L}$ concentrations of volatile organics, and the DAI-GC result probably is more accurate. Sample L23 also contained $200 \mu \mathrm{g} / \mathrm{L}$ of naphthalene. These data are consistent with the elevated TOC in L23 $(558.5 \mathrm{mg} / \mathrm{L})$ compared to the other liquids (49.5 and $59.9 \mathrm{mg} / \mathrm{L}$ for L22 and L119, respectively).

\subsubsection{Sludge}

The two sludges contained PAHs and phthalates at levels of hundreds of milligrams per kilogram. Their SVOA extracts had to be filtered twice because of crystal precipitation. The surrogate standard recoveries of nitrobenzene, 2-fluorophenol, and fleorobiphenyl were low for the soft sludge, but better for the hard sludge. The SVOA extraction for S24 was repeated, using a $\mathrm{pH}$ adjustment to obtain base/neutral and acid fractions. Two organic layers separated when the extracts were concentrated to $1 \mathrm{~mL}$. Also, a lump of dark tar was found in the S24 sample and could be a source of the PAHs that were detected previously. 


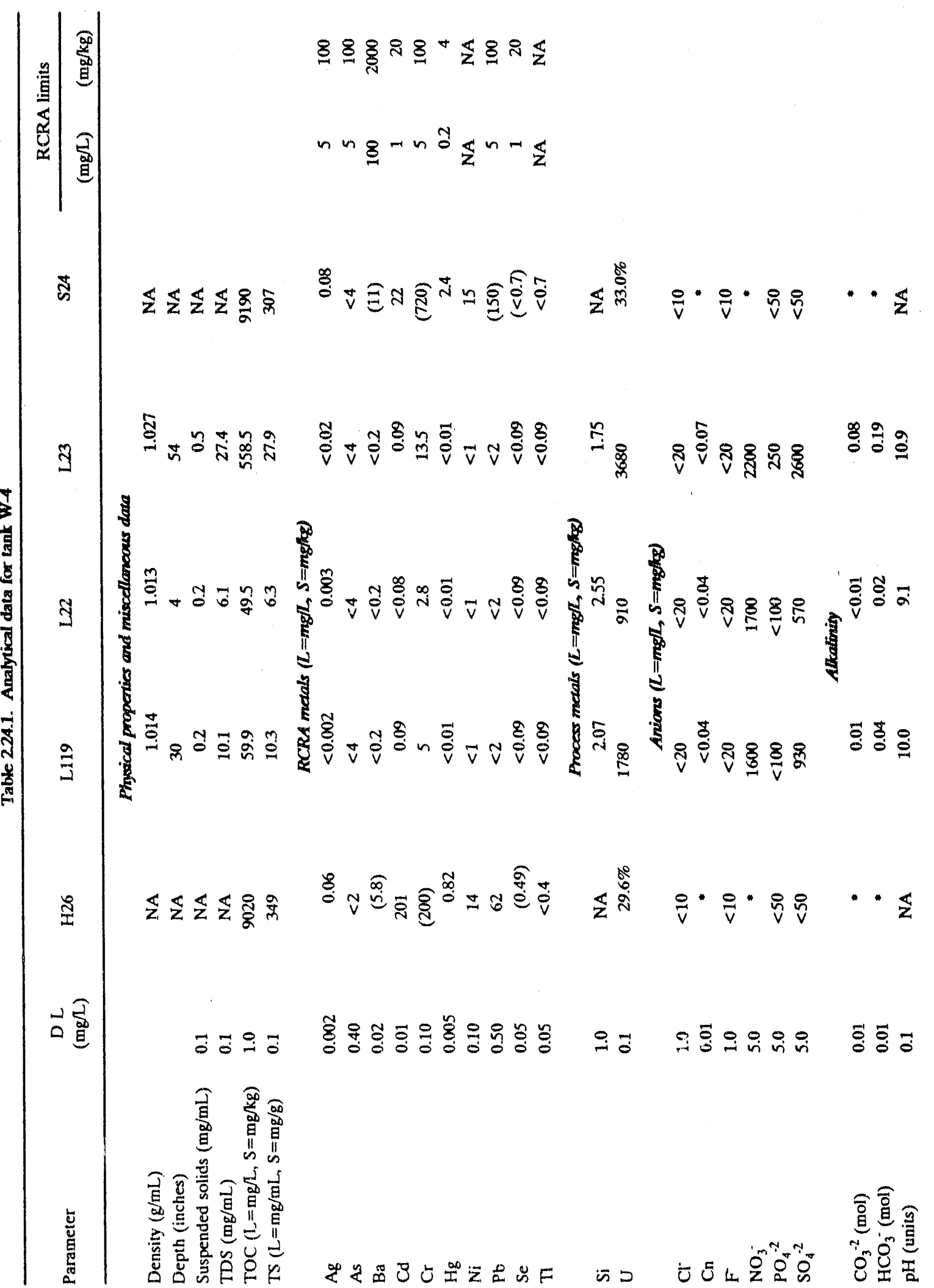




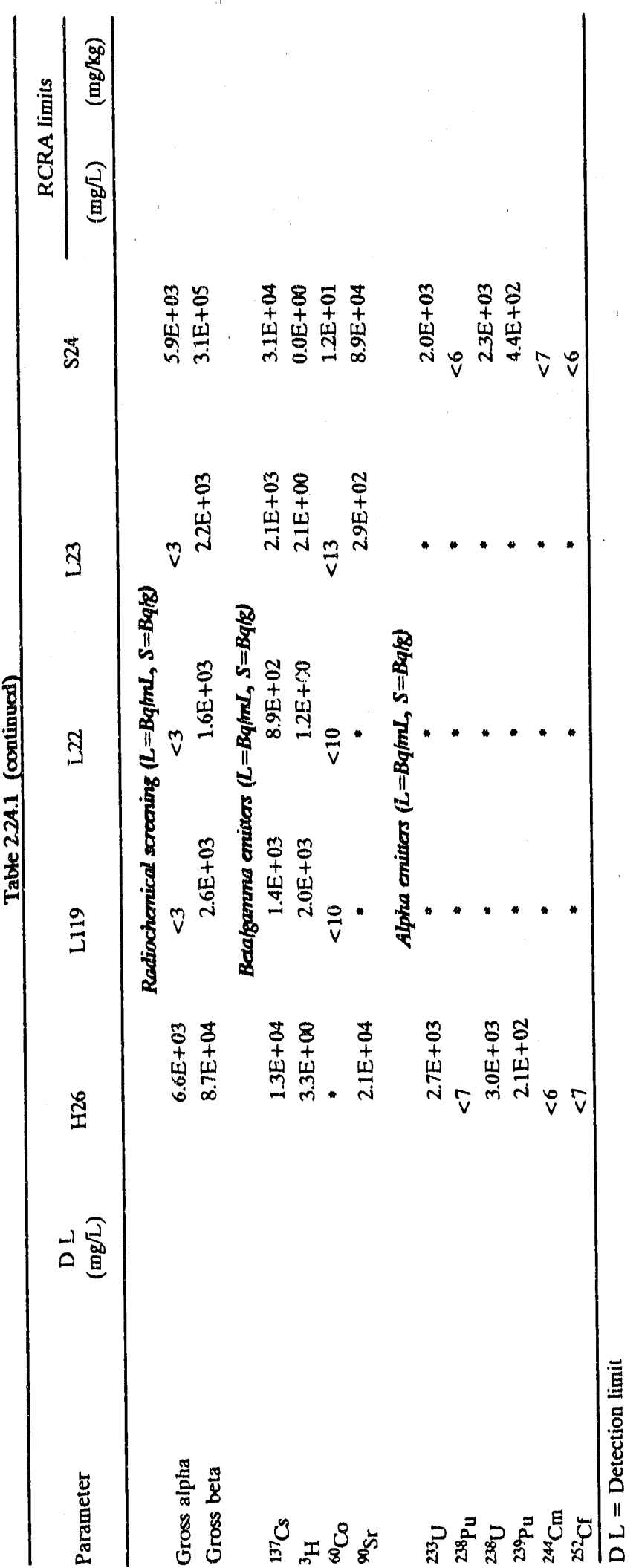




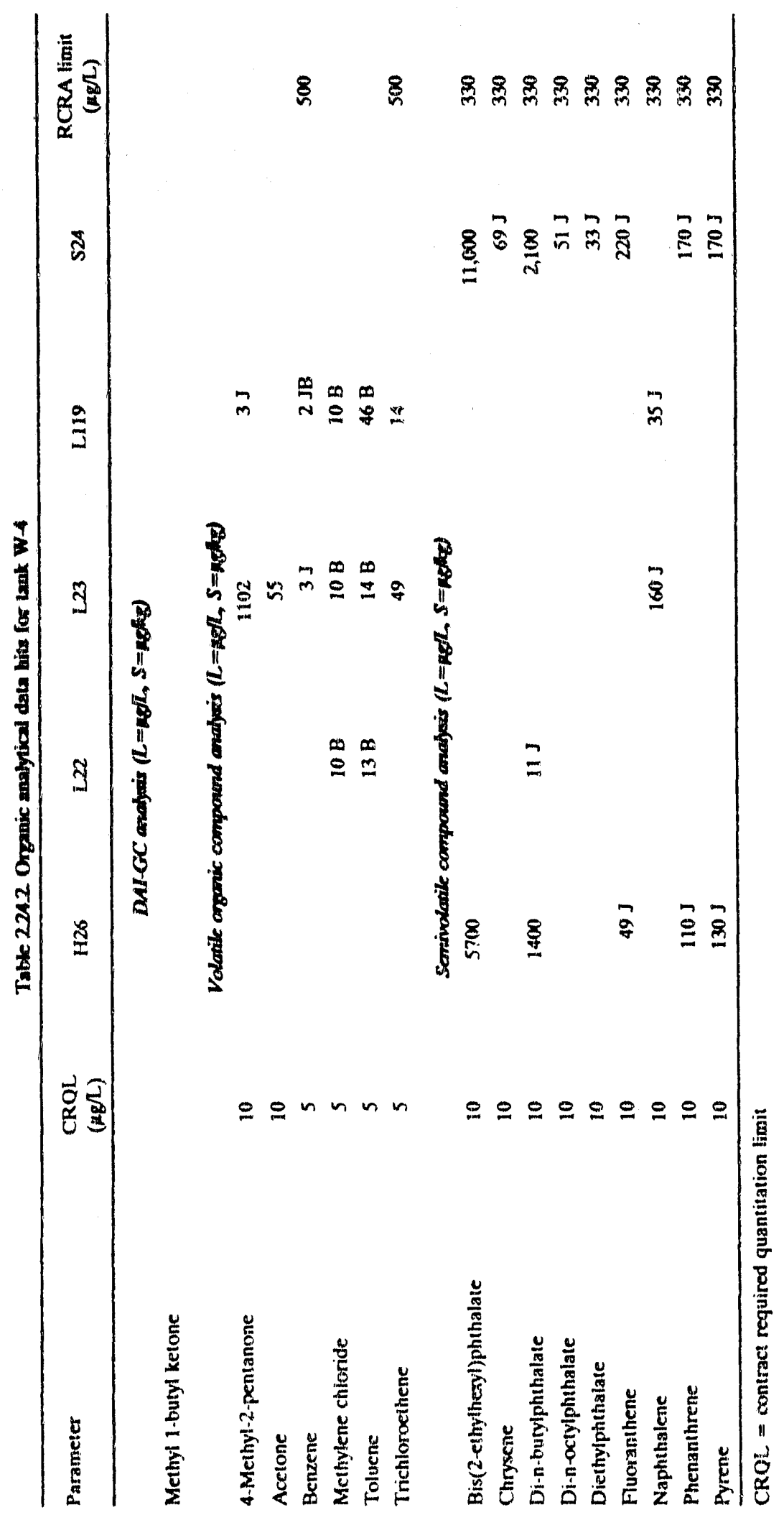




\section{TANK W-13}

\subsubsection{Tank Description}

Tank W-13 is a 2000 -gal stainless steel tank located in the Bethel Valley North Tank Farm. The underground tank was installed in 1945 and received waste from the Radiochemical Processing Facility (Building 3019) and from recovery of fission products.

\subsubsection{Characterization Summary}

The results from this tank are shown in Tables 2.25.1 and 2.25.2.

\subsubsection{Aqueous liquid}

Very little organic matter was determined in these samples. The main compound measured by DAI-GC was methanol. Toluene was the main TCL species found by the VOA. The VOA surrogate standard recoveries were low for $\mathrm{L} 28$, which probably is the reason that the VOA for $\mathrm{L} 27$ yielded more hits. The SVOA did not detect any TCL constituents, and the TICs were not significant, except for TBP $(90 \mu \mathrm{g} / \mathrm{L}$ in L27). The SVOA extracts for both samples were not different from the blank, and L28 was not submitted for GC-MS. The TOCs for these samples were low, 41.4 and $39 \mathrm{mg} / \mathrm{L}$ for $\mathrm{L} 27$ and $\mathrm{L} 28$, respectively. 
Waste Characterization Data for ORNL Inactive LLLW Tanks

Rev. 0 / June 1992

Section 2.25 / page 2 of 3

Table 2.25.1. Anafytical data for tank W-13

\begin{tabular}{|c|c|c|c|c|c|}
\hline \multirow{2}{*}{ Parame:er } & \multirow{2}{*}{$\begin{array}{c}D L \\
(m g / L)\end{array}$} & \multirow{2}{*}{ L27 } & \multirow{2}{*}{ L.28 } & \multicolumn{2}{|c|}{ RCRA limits } \\
\hline & & & & $(\mathrm{m} \mathcal{\ell} \Omega)$ & $(m g / k g)$ \\
\hline \multicolumn{6}{|c|}{ Physical properties and mixcellareous data } \\
\hline Density ( $g / \mathrm{mL}$ ) & & 1.033 & 1.033 & & \\
\hline Depth (inches) & & 4 & 18 & & \\
\hline Suspended solids (mg/mL) & 0.1 & 0.6 & $<0.1$ & & \\
\hline $\mathrm{TDS}(\mathrm{mg} / \mathrm{mL})$ & 0.1 & 34.2 & 33.9 & & \\
\hline $\operatorname{TOC}(\mathrm{L}=\mathrm{mg} / \mathrm{L}, \mathrm{S}=\mathrm{mg} / \mathrm{kg})$ & 1.0 & 41.4 & 39.0 & & \\
\hline $\mathrm{TS}(\mathrm{L}=\mathrm{mg} / \mathrm{mL}, \mathrm{S}=\mathrm{mg} / \mathrm{g})$ & 0.1 & 34.8 & 33.6 & & \\
\hline \multicolumn{6}{|c|}{$R C R A$ metals $\left(L=m g / L_{\nu} S=m g / q_{g}\right)$} \\
\hline A8 & 0.002 & 0.003 & 0.004 & 5 & 100 \\
\hline As & 0.40 & $<4$ & $<4$ & 5 & 100 \\
\hline $\mathrm{Ba}$ & 0.02 & 0.69 & 0.7 & 100 & 2000 \\
\hline $\mathrm{Cd}$ & 0.01 & 0.22 & 0.3 & 1 & 20 \\
\hline $\mathrm{Cr}$ & 0.10 & 2.5 & 2.7 & 5 & 100 \\
\hline $\mathrm{Hg}_{\mathbf{g}}$ & 0.005 & 31 & 35 & 0.2 & 4 \\
\hline $\mathrm{Ni}$ & 0.10 & 8.4 & 8.7 & NA & NA \\
\hline $\mathrm{Pb}$ & 0.50 & 8.8 & 8.9 & 5 & 100 \\
\hline Se & 0.05 & $<0.09$ & $<0.09$ & 1 & 20 \\
\hline $\mathrm{T}$ & 0.05 & $<0.09$ & $<0.09$ & NA & NA \\
\hline \multicolumn{6}{|c|}{ Process metals ( $L=m g / h, S=m g / k g)$} \\
\hline Si & 1.0 & 14.9 & 14.5 & & \\
\hline U & 0.1 & 18.8 & 17.4 & & \\
\hline \multicolumn{6}{|c|}{ Anions $(L=m g / L, S=m g / k g)$} \\
\hline $\mathrm{Cl}^{-}$ & 1.0 & $<3$ & $<3$ & & \\
\hline $\mathrm{Cn}$ & 0.01 & $<0.05$ & $<0.05$ & & \\
\hline $\mathrm{F}$ & 1.0 & $<3$ & $<3$ & & \\
\hline $\mathrm{NO}_{3}^{-}$ & 5.0 & 43 & 43 & & \\
\hline $\mathrm{PO}_{4}^{-2}$ & 5.0 & $<13$ & $<13$ & & \\
\hline $\mathrm{SO}_{4}^{-2}$ & 5.0 & $<13$ & $<13$ & & \\
\hline \multicolumn{6}{|c|}{ Alkalinity } \\
\hline$H^{*}(\mathrm{~mol})$ & 0.01 & 0.26 & 0.27 & & \\
\hline pH (units) & 0.1 & 3.0 & 3.0 & & \\
\hline \multicolumn{6}{|c|}{ Radiochanical screaning $(L=B q / \mathrm{mL}, S=B q / g)$} \\
\hline Gross alpha & & $7.4 E+02$ & $7.6 \mathrm{E}+02$ & & \\
\hline Gross beta & & $1.4 \mathrm{E}+06$ & $1.3 E+06$ & & \\
\hline \multicolumn{6}{|c|}{ Besalgamma anitten $(L=B q / m L, S=B q / g)$} \\
\hline${ }^{137} \mathrm{Cs}$ & & $6.1 \mathrm{E}+05$ & $5.8 \mathrm{E}+05$ & & \\
\hline${ }^{154} \mathrm{Eu}$ & & $1.6 E+03$ & $1.5 E+03$ & & \\
\hline${ }^{3} \mathrm{H}$ & & $2.8 \mathrm{E}+03$ & $2.8 \mathrm{E}+0.3$ & & \\
\hline${ }^{\infty} \mathrm{Co}$ & & $1.5 \mathrm{E}+02$ & $1.9 E+02$ & & \\
\hline $9 \mathrm{Sr}$ & & $5.5 \mathrm{E}+05$ & $5.9 \mathrm{E}+05$ & & \\
\hline \multicolumn{6}{|c|}{ Alpha eminers $(L=B q$, inl, $S=B q$ s $)$} \\
\hline${ }^{238} \mathrm{pu}$ & & $5.5 E+02$ & $5.5 \mathrm{E}+02$ & & \\
\hline${ }^{238} \mathrm{Th} / 232 \mathrm{Th}$ & & $1.2 \mathrm{E}+02$ & $1.3 \mathrm{E}+02$ & & \\
\hline${ }^{239} \mathrm{Pu}$ & & $5.8 \mathrm{E} \cdot 01$ & $6.9 \mathrm{E}+01$ & & \\
\hline${ }^{244} \mathrm{Cm}$ & & $<4$ & $<4$ & & \\
\hline${ }^{252} \mathrm{Cf}$ & & $<4$ & $<4$ & & \\
\hline
\end{tabular}

$\mathrm{DL}=$ Detection limit 
Waste Characterization Data for ORNL Inactive LLLW Tanks Rev. 0 / June 1992

Table 2.252. Organic analytical data hile for tank W-13

\begin{tabular}{|c|c|c|c|c|}
\hline Parameter & $\begin{array}{l}\text { CRQL } \\
(\mu g / L) \\
\end{array}$ & L27 & L.28 & $\begin{array}{c}\text { RCRA limit } \\
(\mu \mathrm{g} \Omega)\end{array}$ \\
\hline \multicolumn{5}{|c|}{$D A I-G C$ malbsis $(L=\mu g / L, S=n g / k g)$} \\
\hline Methyl alcohol & & 11,000 & 5,000 & \\
\hline \multicolumn{5}{|c|}{ Volatile organic compound mabsisis ( $L=\mu g / L, S=\mu g g$ ) } \\
\hline Acetone & 10 & $15 \mathrm{~B}$ & & \\
\hline Benzene & 5 & $3 \mathrm{JB}$ & & 500 \\
\hline Chlorobenzene & 5 & $4 \mathrm{~J}$ & & 100,000 \\
\hline Methylene chloride & 5 & $24 \mathrm{~B}$ & $14 \mathrm{~B}$ & \\
\hline Styrene & 5 & $3 \mathrm{~J}$ & & \\
\hline Toluene & 5 & $100 \mathrm{~B}$ & & \\
\hline
\end{tabular}

$\mathrm{CRQL}=$ contract required quantitation limit 


\section{TANK W-14}

\subsubsection{Tank Description}

Tank W-14 is a 2000-gal stainless steel tank located in the Bethel Valley North Tank Farm. The underground tank was installed in 1945 and received waste from the Radiochemical Processing Facility (Building 3019) and from recovery of fission products.

\subsubsection{Characterization Summary}

The results for this tank are shown in Tables 2.26.1 and 2.26.2.

\subsubsection{Aqueous liquid}

The results were essentially the same as for W-13 (i.e., methanol was the major compound detected by DAI-GC, very little was measured by VOA, and only a phthalate was found by SVOA). As from W.13, the SVOA extract of only one sample (L30) from W-14 was submitted for GC-MS. TBP was estimated at $200 \mu \mathrm{g} / \mathrm{L}$ in L30. The TOCs were higher for this tank (121 and $132 \mathrm{mg} / \mathrm{L}$, respectively). 
Waste Characterization Data for ORNL Inactive LLLW Tanks

Table 226.1. Analytical data for tank W-14

\begin{tabular}{|c|c|c|c|c|c|}
\hline \multirow{2}{*}{ Parameter } & \multirow{2}{*}{$\begin{array}{c}D L \\
(m g / L)\end{array}$} & \multirow{2}{*}{$\mathrm{L} \mathbf{3 0}$} & \multirow{2}{*}{ L31 } & \multicolumn{2}{|c|}{ RCRA limits } \\
\hline & & & & $(\mathrm{mg} / \mathrm{L})$ & $(m g / k g)$ \\
\hline \multicolumn{6}{|c|}{ Physical properties and miscellanoous data } \\
\hline Density (g/mL) & & 1.049 & 1.052 & & \\
\hline Depth (inches) & & 4 & 14 & & \\
\hline Suspended solids (mg/mL) & 0.1 & $<0.1$ & 1.4 & & \\
\hline TDS $(\mathrm{mg} / \mathrm{mL})$ & 0.1 & 29.8 & 27.7 & & \\
\hline $\operatorname{TOC}(L=m g / L, S=m g / k g)$ & 1.0 & 121 & 132 & & \\
\hline$T S(L=m g / m L, S=m g / g)$ & 0.1 & 27.5 & 29.1 & & \\
\hline \multicolumn{6}{|c|}{$R C R A$ meals $(L=m g / L, S=m g / k g)$} \\
\hline As & 2.00 & $<0.002$ & 0.008 & 5 & 100 \\
\hline As & 0.40 & $<4$ & $<4$ & 5 & 100 \\
\hline $\mathrm{Ba}$ & 0.02 & 0.45 & 0.63 & 100 & 2000 \\
\hline $\mathrm{Cd}$ & 0.01 & $<0.2$ & $<0.2$ & 1 & 20 \\
\hline $\mathrm{Cr}$ & 0.10 & 60 & 64 & 5 & 100 \\
\hline $\mathrm{Hg}_{\mathrm{g}}$ & 9.00 & 0.08 & 0.18 & 0.2 & 4 \\
\hline $\mathrm{Ni}$ & 0.10 & 50 & 52 & NA. & NA \\
\hline $\mathrm{Pb}$ & 0.50 & 7.5 & 7.5 & 5 & 100 \\
\hline $\mathrm{Se}$ & 0.05 & $<0.09$ & $<0.09$ & 1 & 20 \\
\hline $\mathrm{T} 1$ & 0.05 & $<0.09$ & $<0.09$ & NA & NA \\
\hline \multicolumn{6}{|c|}{ Process metaly ( $L=m g / L, S=m g / h y)$} \\
\hline Si & 1.0 & 16.5 & 16.2 & & \\
\hline $\mathrm{U}$ & 0.1 & 0.56 & 0.56 & & \\
\hline \multicolumn{6}{|c|}{ Anions ( $L=m g /, S=m g / k g)$} \\
\hline $\mathrm{Cl}$ & 1.0 & $<3$ & $<3$ & & \\
\hline $\mathrm{Cn}$ & 0.01 & $<0.05$ & $<0.05$ & & \\
\hline$F$ & 1.0 & $<3$ & $<3$ & & \\
\hline $\mathrm{NO}_{3}^{-}$ & 5.0 & 70 & 70 & & \\
\hline $\mathrm{PO}_{4}^{-2}$ & 5.0 & $<13$ & $<13$ & & \\
\hline $\mathrm{SO}_{4}^{-2}$ & 5.0 & $<13$ & $<13$ & & \\
\hline \multicolumn{6}{|c|}{ Alkalining } \\
\hline $\mathrm{H}^{+}(\mathrm{mol})$ & 0.01 & 0.19 & $r .21$ & & \\
\hline pH (units) & 0.01 & 0.6 & 0.5 & & \\
\hline \multicolumn{6}{|c|}{ Radiochemical screaning ( $\left.L=B q / m L, S=B q / t^{\prime}\right)$} \\
\hline Gross alpha & & $8.3 \mathrm{E}+03$ & $8.4 E+03$ & & \\
\hline Gross beta & & $9.7 \mathrm{E}+05$ & $1.0 \mathrm{E}+06$ & & \\
\hline \multicolumn{6}{|c|}{ Betalganma emituess $(L=B q \mathrm{~mL}, S=B g / g)$} \\
\hline${ }^{137} \mathrm{Cs}$ & & $4.1 \mathrm{E}+05$ & $4.1 \mathrm{E}+05$ & & \\
\hline${ }^{3} \mathrm{H}$ & & $2.4 \mathrm{E}+\infty 0$ & $3.0 \mathrm{E}+00$ & & \\
\hline${ }^{60} \mathrm{Co}$ & & $1.1 E+03$ & $1.1 E+03$ & & \\
\hline${ }^{90} \mathrm{Sr}$ & & $4.5 \mathrm{E}+05$ & $4.3 E+05$ & & \\
\hline \multicolumn{6}{|c|}{ Alpha animess $(L=B q$ troLL, $S=B q$ /g) } \\
\hline${ }^{238} \mathrm{Pu}$ & & $<150$ & $<150$ & & \\
\hline${ }^{238} \mathrm{Th} / 232 \mathrm{Th}$ & & $8.0 \mathrm{E}+0.3$ & & & \\
\hline${ }^{239} \mathrm{Pu}$ & & $<20$ & $8.2 \mathrm{E}+02$ & & \\
\hline${ }^{244} \mathrm{Cm}$ & & $<100$ & $<50$ & & \\
\hline${ }^{252} \mathrm{Cf}$ & & $\leq 50$ & $<150$ & & \\
\hline
\end{tabular}

$D L=$ Detertion limit 
Waste Characterization Data for ORNL Inactive LLLW Tanks

Table 226.2 Organic anabytical data hits for tank W-14

\begin{tabular}{|c|c|c|c|c|}
\hline Parameter & $\begin{array}{l}\text { CRQL } \\
(\mu g / L)\end{array}$ & L30 & L31 & $\begin{array}{c}\text { RCRA limit } \\
(\mu \mathrm{g} / L)\end{array}$ \\
\hline \multicolumn{5}{|c|}{$D A I-G C$ anabssis $(L=\mu g / L, S=\mu g / / g)$} \\
\hline Ethyl Alcohol & & 3,000 & 2,000 & \\
\hline I-Propyl alcohol & & 2,000 & & \\
\hline Methyl alsohol & & 17,000 & 15,000 & \\
\hline \multicolumn{5}{|c|}{ Volatile onganic compound anabuis $(L=$ mg/h, $S=n g / \mathrm{kg})$} \\
\hline Acetone & 10 & $20 \mathrm{~B}$ & 2513 & \\
\hline Benzene & 5 & $3 \mathrm{JB}$ & $4 \mathrm{JB}$ & 500 \\
\hline Chlorobenzene & 5 & & 6 & 100,000 \\
\hline Methylene chloride & 5 & $2.2 \mathrm{~B}$ & $23 \mathrm{~B}$ & \\
\hline Tetrachloroethene & 5 & & 5 & 700 \\
\hline Toluerie & 5 & $14 \mathrm{~B}$ & $21 \mathrm{~B}$ & \\
\hline Trichloroethene & 5 & & $6 \mathrm{~B}$ & 500 \\
\hline Xylene (total) & 5 & & $4 \mathrm{~J}$ & \\
\hline \multicolumn{5}{|c|}{ Semivolatile compound anabuxis $(L=\mu g / L, S=\mu g / k g)$} \\
\hline Di-n-butylphthalate & 10 & $12 \mathrm{~B}$ & & \\
\hline
\end{tabular}

$\mathrm{CRQL}=$ contract required quantitation ímit 


\subsection{TANK W-15}

\subsubsection{Tank Description}

Tank W-15 is a 2000-gal stainless steel tank located in the Bethel Valley North Tank Farm. The uncierground tank was installed in 1945 and received waste from the Radiochemical Processing Facility (Building 3019) and from recovery of fission products.

\subsubsection{Characterization Summary}

The results for this tank are shown in Tables 2.27.1 and 2.27.2.

\subsubsection{Aqueous liquid}

The results were very similar to those for tanks W-13 and W-14. The DAI-GC indicated $7 \mathrm{mg} / \mathrm{L}$ of methanol for both samples, and VOA detected very little material. No TCL compounds were detected by SVOA. TBP was estimated at $400 \mu \mathrm{g} / \mathrm{L}$ in both L32 and L33. This is consistent with the low TOCs, $53.7 \mathrm{mg} / \mathrm{L}$ for $\mathrm{L} 32$ and $42.7 \mathrm{mg} / \mathrm{L}$ for $\mathrm{L} 33$. The deuterated perylene internal standard was missing from the L32 SVOA extract. The reason is not clear. A matrix effect is possible because the other internal standards were found in the extract, and all were added together before the GC screening. It may be significant that the $\mathrm{pH}$ for both of these samples was 0.2 , which was the lowest of all the inactive tank samples. However, all the internal standards were found in the L33 sample, and the inorganic characterization did not suggest any unusual properties. 
Waste Characterization Data for ORNL Inactive LLLW Tanks Rev. 0 / June 1992

Table 227.1. Anabytical data for tank W-15

\begin{tabular}{lccccc}
\hline \multirow{2}{*}{ Parameter } & $\mathrm{D} \mathrm{L}$ & $\mathrm{L32}$ & $\mathrm{RCRA}$ limits \\
\hline
\end{tabular}

Density (g/mL)

Depth (inches)

Suspended solids ( $\mathrm{mg} / \mathrm{mL}$ )

TDS (mg/mL)

TOC (mg/L)

$\mathrm{TS}(\mathrm{mg} / \mathrm{mL})$

$\mathrm{Ag}$
$\mathrm{As}$
$\mathrm{Ba}$
$\mathrm{Cd}$
$\mathrm{Cr}$
$\mathrm{Hg}$
$\mathrm{Ni}$
$\mathrm{Pb}$
$\mathrm{Se}$
$\mathrm{T}$

Si

U

$\mathrm{Cl}^{-}$

$\mathrm{Cn}$

F

$\mathrm{NO}_{3}{ }^{-}$

$\mathrm{PO}_{4}^{-2}$

$\mathrm{SO}_{4}^{-2}$

$\mathrm{H}^{+}(\mathrm{mol})$

$\mathrm{pH}$ (units)

Gross alpha

Gross beta

${ }^{137} \mathrm{Cs}$

${ }^{3} \mathrm{H}$

${ }^{60} \mathrm{Co}$

${ }^{90} \mathrm{Sr}$

${ }^{233} \mathrm{U}$

${ }^{238} \mathrm{Pu}$

${ }^{236} \mathrm{Th} /{ }^{232} \mathrm{Th}$

${ }^{239} \mathrm{Pu}$

${ }^{244} \mathrm{Cm}$

${ }^{252} \mathrm{Cf}$

$\mathrm{DL}=$ Detection limit
Phosical properties and miscellaneous dosa

$\begin{array}{ccc} & 1.080 & 1.081 \\ 0.1 & 4 & 21 \\ 0.1 & 2.0 & <0.1 \\ 1.0 & 38.1 & 41.1 \\ 0.1 & 53.7 & 42.7 \\ & 40.1 & 38.4\end{array}$

\begin{tabular}{lcccr}
\multicolumn{1}{c}{$R C R A$} & metals & $(R=m g / h, S=m g / k g)$ & & \\
0.002 & $<0.002$ & 0.002 & 5 & 100 \\
0.40 & $<4$ & $<4$ & 5 & 100 \\
0.02 & 3.2 & 3.4 & 100 & 2000 \\
0.01 & $<0.2$ & $<0.2$ & 1 & 20 \\
0.10 & 53 & 55 & 5 & 100 \\
0.005 & 0.40 & 0.39 & 0.2 & 4 \\
0.10 & 48 & 48 & NA & NA \\
0.50 & 7.5 & 7.5 & 5 & 100 \\
0.05 & $<0.09$ & $<0.09$ & 1 & 20 \\
0.05 & $<0.09$ & $<0.09$ & NA & NA
\end{tabular}

\section{Process metals ( $L=m g / L, S=m g / k g)$}

1.0
0.1

22.3

21.9

0.1

387

383

Anions ( $L=m g / L, S=m g / k g$ )

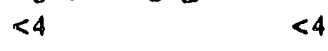

$<0.06<0.07$

$<4<4$

$120 \quad 120$

$<20<20$

$<20<20$

$5.0 \quad<20$

$\begin{array}{lll}0.01 & 0.70 & 0.73\end{array}$

$\begin{array}{lll}0.1 & 0.2 & 0.2\end{array}$

Radiochemical screaning ( $L=B q$ / mLL, $S=B q / g$ )

$$
2.6 \mathrm{E}+03 \quad 2.3 \mathrm{E}+03
$$$$
3.6 \mathrm{E}+06 \quad 3.6 \mathrm{E}+06
$$

Beta/gamma emittess $(L=B q / m L, S=B q / g)$

$1.6 \mathrm{E}+06$

$1.5 \mathrm{E}+06$

$3.0 \mathrm{E}+01 \quad 3.2 \mathrm{E}+01$

$1.4 \mathrm{E}+03 \quad 1.2 \mathrm{E}+03$

$1.7 \mathrm{E}+06 \quad 1.5 \mathrm{E}+06$

Alpha emituers $(L=B q / m L, S=B q t g)$

$\begin{array}{rr}1.1 \mathrm{E}+02 & 1.4 \mathrm{E}+02 \\ 2.5 \mathrm{E}+02 & 2.4 \mathrm{E}+02 \\ 1.5 \mathrm{E}+03 & 1.3 \mathrm{E}+03 \\ 6.3 \mathrm{E}+02 & 5.8 \mathrm{E}+02 \\ <25 & <15 \\ <10 & <10\end{array}$


Waste Characterization Data for ORNL Inactive LLLW Tanks Rev. 0 / June 1992

Table 227.2. Onganic anatytical data his for tank W-15

\begin{tabular}{|c|c|c|c|c|}
\hline Parameter & $\begin{array}{c}\text { CRQL } \\
\mu \mathrm{g} /\end{array}$ & L32 & L33 & $\begin{array}{c}\text { RCRA } \\
\text { limit } \\
(\mu g / L)\end{array}$ \\
\hline \multicolumn{5}{|c|}{$D A J-G C$ analysis $(L=\mu g / L, S=\mu g / k g)$} \\
\hline Methyl alcohol & & 7,000 & 7,000 & \\
\hline \multicolumn{5}{|c|}{ Volatile arganic compound anabusis $(L=\mu g / L, S=\mu g / k g)$} \\
\hline Acetone & 10 & $24 \mathrm{~B}$ & $26 \mathrm{~B}$ & \\
\hline Benzene & 5 & $4 \mathrm{JB}$ & $3 \mathrm{JB}$ & 500 \\
\hline Chlorobenzene & 5 & 5 & $2 \mathrm{~J}$ & 100,000 \\
\hline Methylene chloride & 5 & $21 \mathrm{~B}$ & $24 \mathrm{~B}$ & \\
\hline Styrene & 5 & $2 \mathrm{~J}$ & & \\
\hline Toluene & 5 & $29 \mathrm{~B}$ & $26 \mathrm{~B}$ & \\
\hline Trichloroethene & 5 & $4 \mathrm{JB}$ & $1 \mathrm{JB}$ & 500 \\
\hline Xylene (total) & 5 & $5 \mathrm{~J}$ & $4 \mathrm{~J}$ & \\
\hline
\end{tabular}

$C R Q L=$ contract required quantitation limit 


\subsection{TA NK 7560}

\subsection{Tank Description}

Tank 7560 is a 1000 -gal stainless steel tank located in the Melton Valley area east of the Homogenous Reactor Experiment (HRE) facility (Building 7500). The underground tank was installed in 1957 and served as a waste tank for Building 7500.

\subsection{8 .2 Characterization Summary}

This tank is empty and no samples have been taken. 


\subsection{TANK 7562}

\subsubsection{Tank Description}

Tank 7562 is a 12,000-gal stainless steel tank located in the Melton Valley area southeast of the HRE facility (Building 7500). The underground tank was installed in 1957 and served as a waste tank for Building 7500 .

\subsubsection{Characterization Summary}

The results from this tank are shown in Tables 2.29 .1 and 2.29.2.

\subsubsection{Aquenus liquid}

Very little organic matter was detected in this sample: methanol was detected at $14 \mathrm{mg} / \mathrm{L}$ by DAI-GC, VOA results were very low, and there were no SVOA TCL hits. The TOC was $29.6 \mathrm{mg} / \mathrm{L}$. There was insufficient sludge in S98 for SVOA. 
Waste Characterization Data for ORNL Inactive LLLW Tanks

Rev. 0 / June 1992

Section 2.29 / page 2 of 3

Table 229.1. Anablycal data for tonk 7562

\begin{tabular}{|c|c|c|c|c|c|}
\hline \multirow{2}{*}{ Parameter } & \multirow{2}{*}{$\begin{array}{c}\mathrm{DL} \\
(\mathrm{mg} / \mathrm{L})\end{array}$} & \multirow{2}{*}{$L .97$} & \multirow{2}{*}{$\$ 98$} & \multicolumn{2}{|c|}{ RCRA limits } \\
\hline & & & & $(m g /)$ & $(\mathrm{mg} / \mathrm{kg})$ \\
\hline \multicolumn{6}{|c|}{ Physsical propertier and mincelloneons data } \\
\hline Density (g/mL) & & 1.001 & NA & & \\
\hline Depth (inches) & & 4 & NA & & \\
\hline Suspended solids (mg/mL) & 0.1 & 0.1 & NA & & \\
\hline IDS $(\mathrm{mg} / \mathrm{mL})$ & 0.1 & 0.2 & NA & & \\
\hline $\operatorname{TOC}\left(L=m g / L_{0} S=m g / k g\right)$ & 1.0 & 29.6 & $\cdot$ & & \\
\hline $\mathrm{TS}(\mathrm{L}=\mathrm{mg} / \mathrm{mL}, \mathrm{S}=\mathrm{Lng} / \mathrm{g})$ & C.1 & 0.3 & 75.9 & & \\
\hline \multicolumn{6}{|c|}{ RCRA metals (Lmmg/h, $s$ sumg/ke) } \\
\hline AB & 0.002 & $<0.001$ & 1.0020 & 5 & 100 \\
\hline As & 0.40 & $<2$ & $(<2)$ & 5 & 100 \\
\hline $\mathrm{Ba}$ & 0.02 & $<0$ & 140 & $: 00$ & 2000 \\
\hline $\mathrm{Cd}$ & 0.01 & $<0.09$ & 18 & 1 & 20 \\
\hline $\mathrm{Cr}$ & 0.10 & $<0.1$ & $(2300)$ & $s$ & 100 \\
\hline $\mathrm{Hg}$ & 0.005 & $<0.01$ & 1.8 & 0.2 & 4 \\
\hline $\mathrm{Ni}$ & 0.10 & $<0.6$ & 320 & NA & NA \\
\hline $\mathrm{PQ}$ & 0.50 & $<1$ & 900 & 5 & 100 \\
\hline Se & 0.05 & $<0,05$ & $<0.006$ & 1 & 20 \\
\hline$\pi$ & 0.05 & $<0.05$ & $(<0.006)$ & NA & NA \\
\hline \multicolumn{6}{|c|}{ Propess metals ( $L=m e / h, s=m g / q)$} \\
\hline $\mathrm{Si}$ & 1.0 & 230 & NA & & \\
\hline$U$ & 0.1 & 6.77 & 1990 & & \\
\hline \multicolumn{6}{|c|}{ Amions (L laximg/h, $\left.S=m g / m_{g}\right)$} \\
\hline $\mathrm{Cl}^{\prime}$ & 1.0 & 13 & $<90$ & & \\
\hline $\mathrm{Cn}$ & 0.01 & $<0.05$ & $\bullet$ & & \\
\hline$F$ & 1.0 & $<10$ & $<90$ & & \\
\hline $\mathrm{NO}_{3}$ & 5.0 & 160 & • & & \\
\hline $\mathrm{PO}_{4}^{-2}$ & 5.0 & $<.50$ & $<450$ & & \\
\hline $\mathrm{SO}_{4}^{2}$ & 5.0 & 120 & $<450$ & & \\
\hline \multicolumn{6}{|c|}{ Abratinaty } \\
\hline $\mathrm{HCO}_{3}$ (mol) & 0.01 & $<0.01$ & $\bullet$ & & \\
\hline $\mathrm{pH}$ (units) & 0.1 & 7.4 & NA & & \\
\hline \multicolumn{6}{|c|}{ 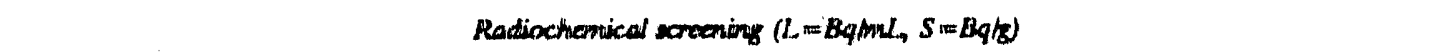 } \\
\hline Grnas alphia & & $<1$ & $<1$ & & \\
\hline Grass beta & & $1.2 \mathrm{E}+0.3$ & $8.8 E+0.5$ & & \\
\hline \multicolumn{6}{|c|}{ 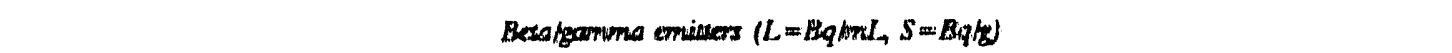 } \\
\hline${ }^{137} \mathrm{Cs}$ & & $1.7 \mathrm{E}+\infty$ & $3.8 E+0.3$ & & \\
\hline${ }^{3} \mathrm{H}$ & & $1.6 \mathrm{E}+00$ & - & & \\
\hline${ }^{2} \mathrm{Sr}$ & & $1.7 E+03$ & $2.2 \mathrm{E}+0.5$ & & \\
\hline
\end{tabular}


Waste Characterization Data for ORNL Inactive LLLW Tanks Rev. 0 / June 1992

Table 2.29.2. Organic analytical dala hite for tank 7562

\begin{tabular}{|c|c|c|c|}
\hline Parameter & $\begin{array}{l}\text { CRQL } \\
(\mu g / L)\end{array}$ & 1.97 & $\begin{array}{c}\text { RCRA } \\
\text { limit } \\
(\mu g / L)\end{array}$ \\
\hline \multicolumn{4}{|c|}{$D A I-G C$ anahysis $(L=\mu g / L, S=\mu g / / g)$} \\
\hline Methyl alcohol & & 14,000 & \\
\hline \multicolumn{4}{|c|}{ Volatile organic compound anabusis $(L=\mu \& / L, S=\mu g / k g)$} \\
\hline Acetone & 10 & $15 \mathrm{~B}$ & \\
\hline Benzene & $s$ & $3 \mathrm{~J}$ & 500 \\
\hline Methylene chloride & 5 & $18 \mathrm{~B}$ & \\
\hline Tetrachloroethene & 5 & $4 J$ & 700 \\
\hline Toluene & 5 & 10 & \\
\hline
\end{tabular}

CRQL = contract required quantitation limit 


\section{TANK T-30}

\subsection{Tank Description}

Tank T-30 is an 825 -gal stainless steel tank located south of Building 4507 in the ORNL Bethel Valley main plant area. The tank was installed in 1961 in an in-ground vault and was used to store radioactive materials for the Curium Recovery Facility (Building 4507).

\subsection{Characterization Summary}

The results from this tank are shown in Tables 2.30.1 and 2.30.2.

\subsubsection{Aqueous liquid}

DAI-GC determined $11 \mathrm{mg} / \mathrm{L}$ of methanol, and very little volatile organics material was found by VOA. SVOA identified only a phthalate. These findings are consistent with the low TOC, $13.7 \mathrm{mg} / \mathrm{L}$. 
Waste Characterization Data for ORNL Inactive LLLW Tanks

Rev. 0 / June 1992

Table 230.1. Analytical data for tank T-30

\begin{tabular}{cccc}
\hline & $\mathrm{DL}$ \\
Parameter & $(\mathrm{mg} / \mathrm{L})$ & $\mathrm{L}$ & RCRA limits \\
\hline
\end{tabular}

Density ( $/ \mathrm{gL})$

Depth (inches)

Suspended solids (mg/mL)

TDS (mg/mL)

TOC (mgl)

TS $(\mathrm{mg} / \mathrm{mL})$

Ag
As
$\mathrm{Ba}$
$\mathrm{Cd}$
$\mathrm{Cr}$
$\mathrm{Hg}$
$\mathrm{Ni}$
$\mathrm{Pb}$
$\mathrm{Se}$
$\mathrm{TI}$

$\mathrm{Si}$

U

$\mathrm{Cl}^{-}$

$\mathrm{Cn}$

$\mathrm{F}$

$\mathrm{NO}_{3}{ }^{-}$

$\mathrm{PO}_{4}^{-2}$

$\mathrm{SO}_{4}^{-2}$

$\mathrm{HCO}_{3}$ (mol)

$\mathrm{pH}$ (units)

Gross alpha

Gross bela

${ }^{137} \mathrm{Cs}$

${ }_{9}^{3} \mathrm{H}$

${ }^{233} \mathrm{U}$

${ }^{238} \mathrm{Pu}$

${ }^{236} \mathrm{U}$

${ }^{234} \mathrm{Pu}$

${ }^{241} \mathrm{Am}$

${ }^{244} \mathrm{Cm}$

${ }^{252} \mathrm{Cr}$
Physical properties and miscellareous daten

1.001

2

$<0.1$

$<0.1$

13.7

$<0.1$

RCRA merals ( $L=m g / L, S=m g / k g)$

$\begin{array}{ll}0.002 & 0.002 \\ 0.4 & <2 \\ 0.02 & <0.1 \\ 0.01 & <0.09 \\ 0.10 & <0.1 \\ 0.005 & 0.01 \\ 0.10 & <0.6 \\ 0.50 & <1 \\ 0.05 & <0.05 \\ 0.05 & <0.05\end{array}$

Process metals $\left(L=m g / L, S=m g / t_{b}\right)$

$1.0 \quad 0.62$

$0.1<1.0$

Anions $(L=m g / L, S=m g / k g)$

$1.0<1$

$0.01<0.06$

$1.0<1$

$5.0<5$

$5.0<5$

$5.0<5$

Alkalinity

$0.01<0.01$

$0.1 \quad 8.7$

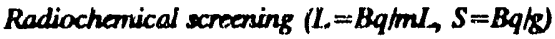

$9.4 \mathrm{E}+01$

$2.7 \mathrm{E}+02$

Bealganma emitters ( $L=B q / m L, S=E K / g$ )

$4.0 \mathrm{E}+00$

$1.6 \mathrm{E}+00$

$1.5 \mathrm{E}+01$

Alphe emittess ( $L=B q$ mon $L, S=B q / g)$

$<2$

$<2$

$4.0 \mathrm{E}+00$

$3.5 \mathrm{E}+00$

$<10$

$3.1 \mathrm{E}+01$

$<2$

$\mathrm{DL}=$ Detection limit 
Waste Characterization Data for ORNL Inactive LLLW Tanks

Rev. 0 / June 1992

Table 230.2. Organic analyical data hits for tank T-30

\begin{tabular}{|c|c|c|c|}
\hline Parameter & $\begin{array}{l}\text { CRQL } \\
(\mu g /))\end{array}$ & L121 & $\begin{array}{c}\text { RCRA limit } \\
(\mu g / L)\end{array}$ \\
\hline \multicolumn{4}{|c|}{$D A I-G C$ anabovis $(L=\pi g / L, S=$ Mg/kg $)$} \\
\hline Methyl alcohol & & 11,000 & \\
\hline \multicolumn{4}{|c|}{ Volatile organic compound arabuis ( $L=m g / L, S=$ ng/kg) } \\
\hline Acetone & 10 & $21 \mathrm{~B}$ & \\
\hline Benzene & 5 & $3 \mathrm{~J}$ & 500 \\
\hline Methylene chloride & 5 & $24 \mathrm{~B}$ & \\
\hline Tetrachloroethene & 5 & 9 & 700 \\
\hline Toluene & 5 & 10 & \\
\hline Trichloroethene & 5 & $4 \mathrm{~J}$ & 500 \\
\hline \multicolumn{4}{|c|}{ Semivolatile compound anabuis ( $L=n g / L, S=n g / \mathrm{kg})$} \\
\hline Bis(2-ethylhexyl)phthalate & 10 & $37 \mathrm{~J}$ & \\
\hline
\end{tabular}

$\mathrm{CRQI}_{2}=$ contract required quantitation limit 


\section{TANK W-19}

\subsection{Tank Description}

Tank W-19 is a 2250-gal stainless steel tank locateci in the ORNL Bethel Valley South Tank Farm area. The underground tank was installed in 1955 and collected waste produced from the recovery and reprocessing of uranium and other nuclear material from the Metal Recovery Facility in Building 3505.

\subsection{Characterization Summary}

This tank is empty and has not been sampled. 


\subsection{TANK W-20}

\section{Tank Description}

Tank W-20 is a 2250 -gal stainless steel tank located in the ORNL Bethel Valley South Tank Farm area. The underground tank was installed in 1955 and collected waste produced from the recovery and reprocessing of uranium and other nuclear material from the Metal Recovery Facility in Building 3505.

\subsection{Characterization Summary}

This tan's is empty and has not been sampled. 


\section{TANK 3001-B}

\subsection{Tank Description}

Tank 3001-B is a 75-gal stainless steel tank located south of Building 3001 under the concrete stairway landing. The tank was installed in 1943 and is believed to have been a holding tank for radioactive laboratory drains in Building 3001.

\subsection{Characterization Summaiy}

A risk characterization has not been performed for tank 3001-B. A waste characterization of the tank contents is under way. A risk characterization will be performed, and the results will be incorporated in a later revision to this data manual. 


\section{TANK 3003-A}

\subsubsection{Tank Description}

Tank 3003-A is a 16,000-gal concrete tank located south of Building 3003 in the Bethel Valley main laboratory area. The tank was constructed in 1943 and collected drainage from three cells and a stack in Building 3003.

\subsection{Characterization Summary}

A risk characterization has not been performed for tank 3003-A. A waste characterization of the tank contents is under way. A risk characterization will be performed, and the results will be incorporated in a later revision to this data manual. 


\section{TANK 3004-B}

\subsection{Tank Description}

Tank 3004-B is a 30-gal stainless steel tank located east of Building 3008 in the Bethel Valley main laboratory area. The tank was installed in an in-grourd vault in 1956 and was used as a waste holding tank for the Low Intensity Test Reactor.

\subsubsection{Characterization Summary}

A risk characterization has not been performed for tank 3004-B. A waste characterization of the tank contents is under way. A risk characterization will be performed, and the results will be incorporated in a later revision to this data manual. 


\subsection{TANK 3013}

\subsubsection{Tank Description}

Tank 3013 is a 400-gal stainless steel tank located south of Building 3013 in the Bethel Valley main laboratory area. The tank was installed ir. 1949 and served an environmental processing laboratory in Building 3013 that dealt with very low-level contaminated environmental samples.

\subsubsection{Characte:ization Summary}

A risk characterization has not been performed for tank 3013. A waste characterization of the tank contents is under way. A risk characterization will be performed, and the results will be incorporated in a later revision to this data manual. 


\section{TANK H-209}

\subsubsection{Tank Description}

Tank H-209 is a 2500-gal (best estimate) stainless steel tank located west of Building 3517 in the Bethel Valley main laboratory area. The tank was installed in 1961 and held condensate and floor drainage from Building 3517.

\subsection{Characterization Sumnary}

A risk characterization has not been performed for tank H-209. A waste characterization of the tank contents is under way. A risk characterization will be performed, and the results will be incorporated in a later revision to this data manual. 
2.38 TANK 7503-A

\subsubsection{Tank Description}

Tank 7503-A is an 11,000-gal stainless steel tank located in an in-ground vault northwest of Building 7503 in the Melton Valley area. The tank was installed in 1962 and was a waste holding tank for the Molten Salt Reactor Experiment.

\subsubsection{Characterization Summary}

A risk characterization has not been performed for tank 7503-A. A waste characterization of the tank contents is under way. A risk characterization will be performed, and the results will be incorporated in a later revision to this data manua:. 


\section{TANK 3001-S}

\subsection{Tank Description}

Tank 3001-S is indicated on drawings to be a 2000-gal stainless steel tank located south of Building 3001 in the Bethel Valley main laboratory area. Its use is not known.

\subsubsection{Characterization Summary}

The location of this tank is unknown. Drawings indicate that a tank was planned to be installed adjacent to the south side of Building 3001. Initial field investigations have not located the tank, and it is possible that the tank was never installed. 
ORNL/ER-80

\section{DISTRIBUTION}

1. L. D. Bates

2. T. W. Burwinkle

3. M. F. P. DeLozier

4. J. T. Etheridge

5. H. R. Gaddis

6. S. B. Garland II

7. C. D. Goins

8. P. J. Halsey

9. J. H. Hooyman

10. L. D. Hyde

11-15. L. L. Kaiser

16. B. L. Kimmel

17. A. J. Kuhaida

18. V. Legg

19-21. D. M. Matteo

22-23. P. T. Owen

24. G. E. Rymer

25. P. A. Schrandt

26. G. W. Sherrill

27. D. W. Swindle

28. D. R. Watkins

29. R. K. White

30. M. L. Whitehead

31. Central Research Library

32-33. Laboratory Records Department

34. ORNL Patent Section

35-39. ER Document Management Center

40. Office of Assistant Manager for Energy Restearch and Development, DOE Oak Ridge Field Office, P.O. Box 2001, Oak Ridge. TN 37831-8600

41. F. H. Edmonds, Radian Corporation, 120 South Jefferson Circle, Oak Ridge, TN 37830

42-47. J. R. Kannard, Prograrn Manager, Bechtel National, Inc., P.O. Box 350, Oak Ridge Corporate Center, 151 Lafayette Drive, Oak Ridge, TN 37830

48-51. R. L. Nace, Department of Energy, Office of Environmental Restoration, Office of Eastern Area Programs, Oak Ridge Program Division, Washington, DC 20585-0002

52-69. R. C. Sleeman, DOE Oak Ridge Field Office, P.O. Box 2001, Oak Ridge, TN $37831-8541$

70-71. J. T. Sweeney, DOE Oak Ridge Field Office, P.O. Box 2001, Oak Ridge, TN 37831 -8541

72-73. Office of Scientific and Technical Information, P.O. Box 62, Oak Ridge, TN 37831 

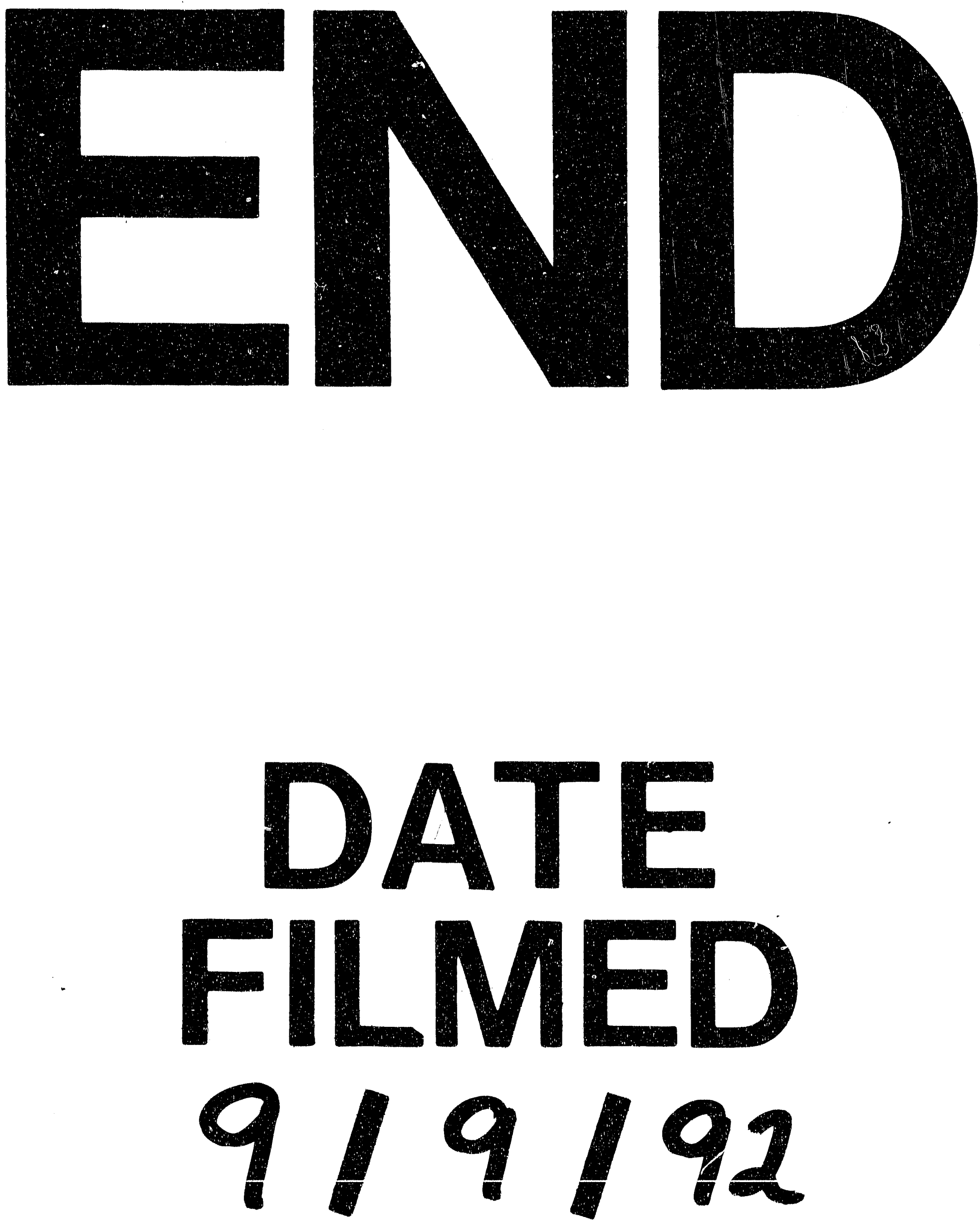
